
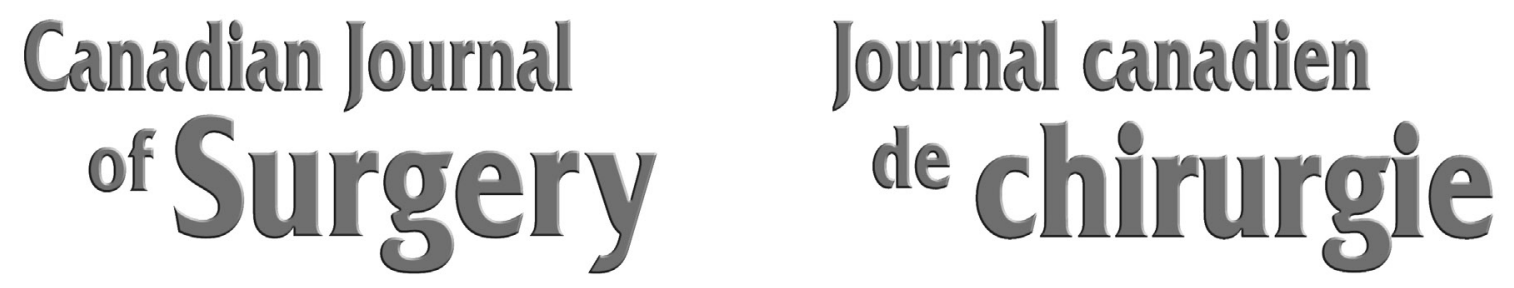

Vol. 62 (3 Suppl 2) June/juin 2019

DOI: $10.1503 /$ cjs.008619

\title{
Trauma 2019
}

Trauma Association of Canada

Annual Scientific Meeting

\section{Westin Calgary \\ Calgary, Alberta}

February 28 to March 1, 2019 
Needs assessment for postgraduate trauma training in general surgery: a qualitative study. Brett Mador ${ }^{1}$, Michael Kim $^{1}$, Fonathan White ${ }^{1}$, Ilene Harris ${ }^{2}$, Ara Tekian'. From the ${ }^{1}$ University of Alberta, Calgary, $\mathrm{AB}$; and the ${ }^{2}$ University of Illinois Chicago, Chicago, IL.

Background: Trauma is considered a key component of surgical training. However, recent changes in practice patterns and training paradigms have resulted in a critical review of curricula for surgical residents. Specifically, a shift toward nonoperative management of traumatic injuries and reduced resident work hours have led to a critical decrease in surgical exposure to trauma. The purpose of this study is to perform a general needs assessment of trauma curricula for general surgery residents across Canada. Methods: The study design included semistructured interviews with trauma education experts across Canada and focus groups with various stakeholder groups. Participants were selected using purposive sampling. The initial interview tool was piloted, and initial data led to modifications in successive iterations. Qualitative analysis was performed using inductive thematic analysis by 2 independent reviewers to identify themes and subthemes. Results: Four trauma education experts participated in the semistructured interviews and 4 separate focus groups with varied stakeholders were conducted. Through inductive thematic analysis, 2 main themes were identified: institutional context and transferability of curricular components. Institutional context was further broken down into subthemes of culture, resources, trauma system and trauma volume. Transferability was applied to the broad categories of trainee outcomes and education strategies. A new conceptual framework was developed to guide ongoing curricular reform for trauma within the context of general surgery training. Conclusion: This general needs assessment for trauma training in Canada has provided valuable data to guide a national curriculum development process. We believe that the framework presented here is also generalizable to other settings using appropriate contextual lenses.

Standardizing concussion recognition and management in Canada: updating the concussion awareness training tool for medical professionals. Pamela Fuselli ${ }^{1}$, Shelina Babul' ${ }^{2}$, Stephanie Cowle ${ }^{1}$, Kate Turcotte ${ }^{2}$. From ${ }^{1}$ Parachute, Vancouver, $\mathrm{BC}$; and the ${ }^{2} \mathrm{BC}$ Injury Research and Prevention Unit, Vancouver, BC.

Background: The Concussion Awareness Training Tool (CATT) is a series of online educational modules and resources with the aim of standardizing concussion recognition, diagnosis, treatment and management. CATT for medical professionals launched in 2013, focusing on recognition and diagnosis, and demonstrating significant positive change in practices $(p=0.001)$ and knowledge $(p=0.039)$. Relaunched in 2018, the new CATT course addresses variations in practice of supporting acute concussion patients. Mobile-friendly versions of the SCAT5 and Child SCAT5 are included. Methods: CATT was redeveloped by BCIRPU and Parachute as part of the Concussion Harmonization Project, supported by the Public Health Agency of Canada. This course targets primary care providers and the variations in practice identified in the literature. The course aligns with the best practice recommendations of the Canadian Guideline on Concussion in Sport, the International Consensus Statement on
Concussion in Sport (Berlin 2016), and clinical practice guidelines and standards developed by the Ontario Neurotrauma Foundation. Results: The priority competencies identified for primary care providers to deliver best practice concussion care were assessing a patient presenting with possible concussion (initial hours postinjury), managing care up to 2-4 weeks postinjury, and identifying when referral to specialized care is required. Inconsistencies in primary practice identified in the literature include use of validated symptom scoring scales, use of diagnostic imaging, recommending and defining cognitive rest, and appropriate application of graduated return to school and sport progressions. Based on that scope, the new CATT MP includes 4 modules: A) Concussion: Definition and Epidemiology; B) Medical Assessment for Concussion, including adjunctive tests; C) Concussion Management and Medical Clearance, including what to inform the patient; and D) Persistent Concussion Symptoms and Management, including making referral decisions. The course is free of charge, available in both English and French, and eligible for credits with the Maintenance of Certification (MOC) program through the Royal College of Physicians and Surgeons of Canada. Conclusion: CATT increases knowledge and awareness of evidence-based concussion recognition, diagnosis, treatment and management to reduce related health problems and the risk of long-term brain damage. The CATT MP is part of the first national effort to harmonize concussion protocols in Canada. Ongoing collaboration among the health, education and sport systems is necessary for concussion outcome optimization.

A combination of midazolam and ketamine for procedural sedation in pediatric emergency medicine department patients to facilitate closed fracture reduction and casting. Deepak Choudhary, Reshvinder Dhillon, Kunal Chadha. From the John R. Oishei Children's Hospital, Buffalo, NY.

Background: Procedural sedation is becoming more common in pediatric emergency medicine (PEM) departments to facilitate closed fracture reduction and casting. However, there is no universally accepted protocol. There are drugs available that are used in variable doses and style. Our objective was to describe the clinical characteristics of a combination of midazolam and ketamine for procedural sedation in PEM department patients presenting with fractures requiring reduction and casting. Methods: This was a retrospective study conducted in the PEM department of a level I trauma centre, Women's and Children's Hospital of Buffalo, New York. Patients were between 2 and 20 years of age who presented to the ED between Mar. 1, 2017, and Aug. 1, 2017, with fractures requiring reduction and casting. Data were collected from electronic medical records. Results: All the reductions were done by orthopedic residents. All sedations were performed or supervised by physicians trained in PEM sedations. Ketamine and midazolam were used in all cases in varied doses. Bag mask with oxygen supply, suction, $\mathrm{TCO}_{2}$ and reversal agent were made available. We excluded patients with a body mass index above 30, nil per os $<3$ hours, poor malampatti and American Society of Anesthesiologists scores, head injury, multisystem trauma and open fractures. Continuous patient monitoring included heart rate, blood pressure, temperature, respiratory rate and and $\mathrm{O}_{2}$ saturations. Patients were monitored for adverse effects. We enrolled 134 patients. All fractures were upper or lower extremities. The time taken for sedation was 10-22 minutes 
for $54.26 \%$ of sedations, $23-37$ minute for $41.86 \%$ and $>37$ minutes for $3.88 \%$. The dose range for midazolam was $<0.05 \mathrm{mg} / \mathrm{kg}$ to $>0.1 \mathrm{mg} / \mathrm{kg}$. The dose range for ketamine was $0.5 \mathrm{mg} / \mathrm{kg}$ to $>2 \mathrm{mg} / \mathrm{kg}$. Ten percent of patients experienced mild nausea and $1 \%$ had emesis. None of our patients experienced aspiration or lethargy or required any reversal agents or intubation. All patients were stable, improved, alert and oriented at the time of discharge. The increasing dose of midazolam was found to be associated with increased use of respiratory support (Pearson $\chi^{2}, p=0.047$ ). Conclusion: The combination of midazolam and ketamine provides effective procedural sedation and analgesia for fracture reduction and casting in PEM patients and appears to be safe. Even though respiratory adverse effects were documented in a significant number of patients, they all were brief and improved with airway positioning, suction, face mask or nasal cannula $\mathrm{O}_{2}$. Similarly, the heart rate and blood pressure variability was documented in several patients; they all were short lasting and required no intervention.

The chaos of triage: a model for early exclusion of heart injury in chest gunshot wound patients. Faran Bokbari, Chib-Yuan Fu, Francesco Bajan, Stanley Welsh, Matt Kaminsky, Andrew Dennis, Fredric Starr, Caroline Butler, Thomas Messer, Stathis Poulakidas. From the Cook County Hospital, Chicago, IL.

Background: Heart injury requires prompt diagnosis and treatment. In certain cases, cardiac ultrasonography has low sensitivity for detecting cardiac injury. We evaluated independent factors for heart injury that could be obtained during the prehospital or triage phase only. Methods: The US National Trauma Data Bank was queried for chest gunshot wound (GSW) patients treated between July 1, 2009, and June 30, 2016. Patients with and without heart injuries (ICD-9 codes 861.00-03, 861.10-13) were analyzed. Multivariate logistic regression was performed to evaluate independent factors for heart injury. Registry data from the Stroger Hospital of Cook County (July 1, 2016, to June 30, 2017) were used for external validation. Results: A total of 47044 chest GSW patients were evaluated; 4047 (8.6\%) patients had heart injuries. The mortality associated with chest injury with and without cardiac injury was $21.7 \%(879 / 4047)$ and $9.0 \%$ (3864/42997), respectively. Patients with heart injuries were younger (26.4 v. $29.3 \mathrm{yr}, p<0.001)$, had lower systolic blood pressure (34.7 v. $103.8 \mathrm{~mm} \mathrm{Hg}, p<0.001)$ and Glasgow Coma Scale (GCS) scores (5.1 v. 11.2, $p<0.001)$ on presentation in the emergency department, higher apnea rates $(58.3 \%$ v. $14.7 \%, p<0.001)$, higher rates of pulselessness $(59.9 \%$ v. $12.0 \%$, $p<0.001)$ and more suicide intent $(9.7 \%$ v. $8.5 \%, p<0.001)$ than patients without heart injuries. The heart injury prediction model had a high specificity (88.8\%) when age, systolic blood pressure, GCS score, apnea, lack of pulse and suicide intent were included. External validation with the local database showed high specificity (95.6\%). Conclusion: Our model has high specificity and can be beneficial for early triage of cardiac injury in chest GSW patients.

P.A.R.T.Y. PROGRAM 2.0: updating current injury prevention education to match 21 st century student expectations. Brandy Tanenbaum, Sharon Ramagnano. From the Sunnybrook Health Sciences Centre, Toronto, ON.

Background: The Prevent Alcohol and Risk-related Trauma in Youth (P.A.R.T.Y.) Program was established at Sunnybrook
Health Sciences Centre in 1986 to address teenage curiosity about trauma patient pathways. The Toronto program operates twice weekly and serves approximately 2500 high school students each school year. In early 2018, the P.A.R.T.Y. headquarters underwent an internal quality improvement review to identify gaps in content relative to current literature, injury prevention trends, and revisions to the Ontario Public Health standards. Methods: A consultative and facilitated program review was used to engage with stakeholders and identify opportunities for change and improvement. Several meetings were held, leading to a summative facilitated advisory meeting in July 2018 with guest speakers from organizational development and youth psychiatry. This meeting provided a venue for program staff and volunteers to understand the needs of students in the 21 st century learning environment and what that means in an injury prevention context. Results: The consensus developed throughout the change process created space and comfort to revise both content and format. The primary areas of concern that were addressed in this process included 1) didactic teaching, 2) vicarious trauma, 3) too much sitting and 4) lack of skill-based learning. The program format and schedule of the day were shifted to include more opportunities for student engagement and "teach back" by recognizing that knowledge can be found among the students. Program content that focused solely on graphic injury images and stories of severe injury were replaced by stories of hope and resilience following traumatic injury. Building on the thread of resilience, there are opportunities throughout the program for students and speakers to talk about mental health support and personal strategies for managing stress in stressful jobs. The agenda was revised to allow time for movement and reflection exercises that included drama and role-playing. Last, the program intentionally created opportunities for skill-based learning, including how to perform the recovery position, how to call 911 , and how to administer naloxone. The overarching themes of the program are hope, resilience and empowerment. Students are left feeling confident in their ability to effect change in health and injury outcomes in their communities, their personal network of friends and family and for themselves. Conclusion: By using evidence-based quality improvement strategies, program staff and stakeholders were able to maintain the core values of the P.A.R.T.Y. Program while creating relevance for today's youth. A formal evaluation of the programming changes will begin shortly, the results of which will be available in 2019. Evidence will be shared with all Canadian and international P.A.R.T.Y. Program licence holders, and new program standards will be developed to include new content.

The first Canadian ER-REBOA experience: using a systemic team-based approach. Nancy Tze ${ }^{1}$, Jeremy Grushka ${ }^{2}$, Andrew Beckett $^{1,2}$, Carole Filteau ${ }^{1}$, fosée Larocque ${ }^{1}$. From the ${ }^{1}$ McGill University Health Centre, Montreal, QC; and ${ }^{2} \mathrm{McGill}$ University, Montreal, QC.

Background: Resuscitative endovascular balloon occlusion of the aorta (REBOA) is a relatively new device designed to temporarily occlude large vessels in support of hemorrhage control. Although the device has been widely used in the United States, Japan and in military settings, it was approved for use by Health Canada only in October 2017. The Montreal General Hospital, a level I academic trauma centre is the first hospital in Canada to implement and use the ER-REBOA successfully. Methods: We describe 
how new technology was introduced and put into practice through a multidisciplinary team approach. Trauma team leaders (TTLs) were trained in the insertion of the ER-REBOA, and nurses were trained on its use and set-up. Important issues addressed during the process include funding and procurement parameters for the device and ancillary equipment, creation of a REBOA kit, protocol development, training requirements, and the identification of the facilitators and barriers to implementation. Results: An integrated approach to introduce a novel technology began in May 2017 in collaboration with trauma surgeons, TTLs, emergency physicians, interventional radiology, anesthesiology, and emergency department (ED) and operating room (OR) nurses. Funding for the ER-REBOA was made possible through the MGH Foundation. The first ER-REBOA was successfully inserted on May 10, 2018, in the OR. A formalized training structure was universally agreed upon by the team. A hands-on simulation session $(1.5 \mathrm{~h}$ ) was provided by the company representative to TTLs, nurse educators, and nursing leadership. The TTLs were mandated to attend a certified REBOA training course, followed by validation with an in-house interventional radiologist. ED nurses were trained on arterial line set-up, patient surveillance, and documentation (total number of nurses trained: 40/90 total RNs, 40/50 TTLs and, 45/50 nurses-in-charge). OR personnel were oriented with a slide presentation. From May to October 2018, the ER-REBOA has been inserted in 5 patients (60\% male and $40 \%$ female, with balloon deployment in $4 / 5$ patients) and attempted in 2 patients. All 7 patients sustained blunt trauma; 1 patient sustained a gunshot wound to the lower extremity. In all successful cases, ER-REBOA was performed in the OR (all with ultrasound and fluoroscopic guidance). All 5 inserted REBOAs were positioned in Zone 3. There were no early deaths or complications in the 4 patients who underwent successful ER-REBOA insertion. Conclusion: The ER-REBOA is a valuable adjunct for improving hemodynamic status in patients with traumatic noncompressible torso hemorrhage. Implementation of the ER-REBOA using a strategic, organized multidisciplinary team approach is an effective strategy for introducing a new technology. Financial considerations and identifying sources of recurrent funding; maintaining competency for a low-frequency, high-risk procedure; and continuous quality improvement are challenges that must be addressed to ensure the sustainability of the ER-REBOA in the future.

Blunt injury in the older adult: baseline frailty characteristics of a prospective cohort study. Ting Hway Wong ${ }^{1}$, Nivedita Nadkarni ${ }^{2}$,Wei Chong Chua ${ }^{3}$, Lynette Loo ${ }^{4}$, Arron Seng Hock Ang $^{5}$, Philip Tsau Choong Iau ${ }^{4}$, Jerry Tiong Thye Goo ${ }^{6}$, Kim Chai Chan ${ }^{7}$, Tian Natasha Adam ${ }^{1}$, Dennis Chuen Chai Seow", Yee Sien Ng ${ }^{1}$, Rabul Malbotra ${ }^{2}$, Angelique Wei-Ming Chan', David Bruce Matchar ${ }^{2}$, Hai Van Nguyen ${ }^{8}$, Marcus Eng Hock Ong$^{1}$. From the 'Singapore General Hospital, Singapore; the ${ }^{2}$ Duke-National University of Singapore, Singapore; the ${ }^{3}$ Tan Tock Seng Hospital, Singapore; the ${ }^{4}$ National University Hospital, Singapore; the ${ }^{5}$ Changi General Hospital, Singapore; the ${ }^{6}$ Khoo Teck Puat Hospital, Singapore; the ${ }^{7} \mathrm{Ng}$ Teng Fong General Hospital, Singapore; and the ${ }^{8} \mathrm{Memorial}$ University Newfoundland School of Pharmacy, St. John's, NL.

Background: Frail patients are more likely to have poor outcomes and increased health care costs. Our retrospective data suggest that patients who suffer an injurious, low fall are more likely to be frail preinjury than patients injured by other highervelocity mechanisms of blunt injury. We present some of the baseline frailty characteristics of our prospective cohort study of older Singaporeans hospitalized for injury related to the most common mechanisms of injury, falls and motor vehicle injuries. Methods: Patients aged 55 and older, admitted for $>48$ hours after blunt injury, with an Injury Severity Score (ISS) or new ISS $>9$, or an Organ Injury Scale score $\geq 3$ were recruited at the initial hospitalization after screening using National Trauma Registry data. For patients unable to give consent owing to preexisting conditions (e.g., dementia) or as a result of their injury (e.g., head injury), caregivers were interviewed as surrogates of preinjury status. Results: There were 208 patients recruited: 120 men and 88 women. Most (154, 70.4\%) were injured by low fall, $44(21.2 \%)$ by motor vehicle injury, and 10 (4.8\%) from fall $>0.5 \mathrm{~m}$. Median age was 75 (interquartile range [IQR] 66-83) years, with low fallers being the oldest (median $77 \mathrm{yr}$ ), followed by high-fallers (median $74 \mathrm{yr}$ ); motor vehicle-injured patients were the youngest (median $63 \mathrm{yr}$ ). Median ISS was similar for low-fallers (13, IQR 10-17) and motor vehicle-injured patients (14, IQR 10-22), and higher for the high-fallers (20, IQR $10-27)$. The most common admission discipline was neurosurgery $(91,43.8 \%)$, followed by orthopedics $(42,20.2 \%)$, with similar proportions for low- and high-fallers, whereas the motor vehicle-injured patients were mostly admitted to general surgery $(21,47.7 \%)$. From the questionnaire, survey items easily answered by surrogates had a higher completion rate. Weight loss was reported by a higher proportion of low-fallers (41, $26.6 \%)$ than high-fallers $(1,11.1 \%)$ and motor vehicle-injured patients $(6,13.6 \%)$. Preinjury walking difficulties were reported by a higher proportion of low-fallers $(51,33.1 \%)$ than highfallers $(2,20.0 \%)$ and motor vehicle-injured patients $(3,6.8 \%)$. Of the patients able to complete the Mini-Mental State Examination and grip strength measurement by Jamar dynamometer, low-fallers had the worst scores, while motor vehicle-injured patients had the best scores. Conclusion: Despite similar severity of injury, patients admitted after an injurious, low fall manifest more premorbid or baseline frailty-related features than patients admitted after motor vehicle injuries, while high-fallers are in between. Future follow-up data from our study should shed light on whether these differences increase over time after discharge, and what interventions might be considered for highrisk patients.

Use of radiologic grading systems for splenic injuries: effects of Injury Severity Score and management. Ian Grant $^{1,2}$, Facinthe Lampron ${ }^{2}$, Alexandre Bougie ${ }^{1,2}$. From the ${ }^{1}$ University of Ottawa, Ottawa, ON; and ${ }^{2}$ The Ottawa Hospital, Ottawa, ON.

Background: Accurate diagnosis and management of injuries is essential for multisystem trauma patients. Splenic injuries are reliably identified and treated; however, accurate American Association of Surgery for Trauma (AAST) grading of the injuries is inconsistent. Decisions for management are made based on patient characteristics, but also the grade of the injury. The goal was to determine frequency of AAST grading of splenic injuries and the effect grading has on the reported Injury Severity Scores (ISS) and management. Methods: A prospectively collected 
registry was used to identify patients who presented to a Canadian level I trauma centre between 2015 and 2018 with a splenic injury. Radiology reports were surveyed for descriptions of the injury and whether AAST or other grading systems were used. All images were then reviewed by 2 trauma surgeons and given an AAST grade. Management strategy for the injury and whether the new AAST grading affected the ISS were reviewed. Results: A total of 124 patients with splenic injuries were admitted over the study period. Twenty-two patients were excluded because imaging was not available. Only $40 \%(n=41)$ of splenic injuries were graded by radiology in final reports. In this cohort, higher AAST grade correlated with increased need for intervention (laparotomy or embolization): 13\% grade I, 16\% grade II, $30 \%$ grade III, $90 \%$ grade IV, and $67 \%$ grade $\mathrm{V}$. When we compared those that were assigned a grade by the radiologists to those that were not, the frequency of intervention did not change statistically. Independent review of all scans identified that $34 \%(n=35)$ of patients had a change in their ISS by an average of 5.9 after applying the AAST grading system to their scans; $15 \%(n=15)$ had their ISS increase, whereas 20\% $(n=20)$ had their ISS decrease. Most frequently, the Abbreviated Injury Scale (AIS) score increased or decreased by only 1 point; however, 1 patient had an increase of 2 points on the AIS, and 1 patient had a decrease of 2 points on the AIS. Conclusion: The infrequent use of an accepted scheme for the description of splenic injuries leads to inconsistency in reporting injuries and inaccuracy of ISS scores, decreasing the quality of the data collected. Decision to intervene on injuries is related to the severity of the injury. Implementation of a standardized report for radiology may improve interprofessional communication and favour consistency in the treatment of splenic injury.

The incidence, risk factors and outcomes of atrial fibrillation and its treatment in the surgical intensive care unit. Saad Sabi ${ }^{1}$, Carlos Brown ${ }^{2}$, Anish Patel, Leonard Edwards ${ }^{1}$, Kate Spitz ${ }^{1}$. From the ${ }^{1}$ Dell Medical School at The University of Texas at Austin, Austin, TX; and the ${ }^{2}$ Dell Seton Medical Center at the University of Texas, Austin, TX.

Background: Atrial fibrillation (AF) is an atrial arrhythmia that can lead to a rapid ventricular response, hypotension and even death. The incidence, risk factors and outcomes for AF in the adult trauma patient are not well known. Moreover, there is little to no evidence to suggest which treatment for AF may be most effective. Methods: We performed a retrospective study (20102017) of all adult trauma patients admitted to our surgical intensive care unit (ICU) at an urban, academic level I trauma centre. Patients with and without $\mathrm{AF}$ were compared using univariate and multivariate analysis. Treatment for AF included diltiazem, $\beta$-blockers and amiodarone. Results: There were 4214 trauma patients admitted to our trauma centre, 73 (2\%) of whom developed AF after admission to the hospital. Patients with AF were older, more often male and white, and more often sustained blunt trauma. Independent risk factors for AF included older age (odds ratio $1.1, p<0.0001$ ), male sex (odds ratio $2.7, p=0.001$ ), and higher Glasgow Coma Scale (GCS) score (odds ratio 1.1, $p=$ 0.04). The most common initial treatment for $\mathrm{AF}$ included diltiazem $(70 \%, n=51)$, $\beta$-blockers $(15 \%, n=11)$, and amiodarone $(15 \%, n=11)$. As an initial single agent, diltiazem was more effective (29\%) for rate and rhythm control than $\beta$-blockers (9\%) and amiodarone $(0 \%)(p=0.02)$. Conclusion: AF occurs in $2 \%$ of adult trauma patients admitted to the surgical ICU. Independent risk factors for AF included age, male sex and GCS score. Diltiazem may be the most effective initial treatment for rate and rhythm control of AF in this population.

Risky play: For the sake of the kids, can we accept play that includes the possibility of injury? Brandy Tanenbaum, Sharon Ramagnano. From the ${ }^{1}$ Sunnybrook Health Sciences Centre, Toronto, ON.

Background: More children are injured falling out of beds than out of trees each year, yet every day communities find ways of limiting access to outdoor play, physical activity and the opportunity to develop lifelong skills. The consequence of a childhood stripped of the opportunity to explore risk in play includes decreased motor and spatial skills, decreased self-esteem and unhealthy risk-taking in adolescence with respect to substance use, relationships and sexual behaviour. Methods: The Outdoor Play Position Statement, published in 2015, supports active outdoor play that includes age- and stage-appropriate and dosed exposure to elements of risk and the possible outcome of injury. Despite this publication, there has been scant discussion among injury-prevention practitioners to reconcile the tension between injury and play. As a result, there is no clear message from this sector on the subject. Results: With modern science and advances in public health and health care delivery, life expectancy significantly improved. However, this increase has stopped, and children today are not expected to live as long as their parents' generation. Something has changed in the experience of childhood that profoundly affects the health and wellbeing of today's young people. In the most recent publication of the Ontario Student Drug Use and Health Survey, 39\% of students indicate a moderate-to-serious level of psychological distress (symptoms of anxiety and depression). In the most recent ParticipACTION Report Card, 35\% of 5- to 17-year-olds meet the physical activity recommendation within the Canadian 24-Hour Movement Guidelines for Children and Youth. Onethird of Canadian children and adolescents have at least 1 risk factor for metabolic syndrome. While there is a growing spotlight on childhood falls and preventing emergency department visits and hospitalizations, there is concurrent emphasis on active and risky play that includes the possibility of "learning injuries." The inherent opposition between play and injury creates tension among physical activity promotion and injury prevention practitioners. How can these conflicting objectives reconcile themselves in order to foster healthy and safe community experiences and consistent messaging from injury prevention, public health and health care agencies? Conclusion: In a time when children are falling below recommended guidelines for physical activity and this failure contributes to exponential risk for chronic disease and injuries, it is prudent to consider alternative policy, messaging and programming. This presentation highlights key concepts, literature and resources related to risk, play and injuries with the hope of sparking conversation among health care professionals and injury prevention practitioners.

Effectiveness of mindfulness-based interventions in adult trauma patients - a systematic review. Sonshire Figueira ${ }^{1}$, Facintbe Lampron ${ }^{1}$, Miguel Nucete ${ }^{2}$, Sikora Lindsey ${ }^{3}$. From 
the ${ }^{1}$ The Ottawa Hospital, Ottawa, ON; ${ }^{2}$ Inside Therapy Ottawa, Ottawa, ON; and the ${ }^{3}$ University of Ottawa, Ottawa, ON.

Background: Every day, more than 10000 Canadians receive medical attention for serious traumatic injuries. Given the severity and complexity of their injuries, trauma patients frequently experience psychological stress during their hospitalization. Mindfulness-based interventions (MBIs) are a widely recognized way to improve stress and well-being. The aim of this systematic review is to evaluate the effectiveness MBIs among adult trauma patients. Methods: A systematic literature review was conducted using Embase, Medline, ERIC, Cochrane, PsycINFO and CINAHL from the date of inception to October 2018. There were no language exclusion criteria, nor any other publication restrictions. Two reviewers independently reviewed selected studies. Disagreements will be adjudicated by a third reviewer. Articles were included if they were peer-reviewed and reported data about MBIs for adult trauma patients. Analysis restricted to descriptive findings. The PRISMA guideline was followed. PROSPERO, \# CRD42018100483. Results: We identified 3953 articles with the initial search strategy. Reviewers will extract data, check accuracy, and assess risk of bias and quality (sample size, bias and validation techniques). This systematic review will describe the characteristics (population, intervention type, outcome measures) and summarize evidence regarding MBIs for the adult trauma population. Conclusion: The number of published studies evaluating effectiveness of MBIs in trauma patients is very limited. However, there may be some benefits of exposing patients to MBIs in terms of mental and physical health. Further research needs to take place in order to determine how MBIs may benefit the adult trauma patient population.

Impact of autopsy reports on Injury Severity Score calculation - a quality-improvement initiative. Heather Knight, Facinthe Lampron, Sonshire Figueira. From the Ottawa Hospital, Ottawa, ON.

Background: The Injury Severity Score (ISS) is a composite anatomic scale that correlates with morbidity and mortality; it is calculated primarily through the injuries reported in diagnostic imaging. In cases where the patient dies before diagnostic imaging can be performed, autopsy reports may play a vital role in augmenting the list of injuries by filling in the missing information. The objective of this study is to determine the impact of autopsy reports on the accuracy of ISS calculation. Methods: We retrospectively analyzed prospectively collected data from a level I trauma centre database. Autopsy reports were requested from the Regional Coroner Office. Inclusion criteria were consecutive patients from July 2016 to September 2018 who died in the emergency department (ED) due to traumatic injuries before any diagnostic imaging being done. The ISS was calculated before obtaining autopsy reports and then recalculated after receiving them. Comparative analysis was done to document the extent of the variation. Results: Forty-five patients were included in the study. Thirteen (28\%) autopsy reports were received. Seven were excluded for homicide. Overall, the mean ISS before access to the autopsy report was lower than the mean ISS after receiving autopsy reports (9.2 v. 30.5). There were
7 deaths after a trauma team activation with a mean ISS of 12.6 before access to the autopsy report versus 31.6 after receiving the autopsy report. Cases without trauma team activation $(n=3)$ had a mean ISS of 4 before the autopsy report versus 27.3 after the autopsy report. Three autopsy reports concluded that trauma was not the cause of death. The overall ISS of deceased trauma patients for the studied period was 23.9 before versus 24.9 after the addition of autopsy report information. Conclusion: Having access to autopsy reports significantly increases the accuracy of ISS calculation for trauma patients who die before any diagnostic imaging. It increases the overall ISS for deceased patients from 23.9 to 24.9 . As a result, accuracy of the trauma database will positively impact the quality of research and performance improvement projects and inform better administrative decisions.

High-velocity motor vehicle collisions with seatbelt sign should raise the index of suspicion for hidden abdominal injuries. Ian Grant, Maber Matar, D'Elia Michael. From The Ottawa Hospital and University of Ottawa, Ottawa, ON.

Background: Hollow viscus injuries after blunt abdominal trauma are difficult to identify and contribute to significant morbidity. Here we describe a patient involved in a high-speed motor vehicle crash (MVC) who had no clear evidence of hollow viscous injury on computed tomography (CT) scan, but intraoperatively was found to have 4 significant small bowel injuries and a colonic devascularization. Methods: Hollow viscus injuries are rare, and difficult to assess. A 24-hour delay in identifying these injuries can increase mortality from $2 \%$ to $30 \%$. A seatbelt sign in the presence of a significant MVC has been associated with up to $10 \%$ presence of a hollow viscus injury. Furthermore, modest increases in speed have been shown to be associated with significant risks of intra-abdominal injury. These are key factors in assessing MVC patients. Results: Belted in a 3-point restraint, a middle-aged man was involved in a head-on MVC at $80 \mathrm{~km} / \mathrm{h}$, and was transferred to a level I trauma centre. Physical examination showed a seatbelt sign, with abdominal tenderness, along with multiple orthopedic injuries. Initial heart rate was 120 , but he was responsive to fluid, permitting a CT scan. CT demonstrated moderate hemoperitoneum, a contrast blush from the small bowel mesentery, but no solid organ injury. Intraoperatively, the patient had significant hemoperitoneum with clots. A complete transection of the terminal ileum associated with a mesenteric bleed from the cut edge was identified. There were 2 additional blow-out type injuries of the anti-mesenteric aspect of the jejunum, and a bucket-handle injury of the proximal ileum. The right, transverse and descending colon was normal, but a bruise on the distal sigmoid was identified. After complete mobilization of the sigmoid, a bucket-handle injury of the rectosigmoid junction was discovered. The patient was hemodynamically stable, and there were no other injuries identified. Primary repair of the 2 blow-out injuries and resection with primary anastomosis of the remaining injuries was carried out, and the patient was brought to the intensive care unit. Conclusion: Abdominal CT scan has poor sensitivity for small bowel injuries. In the presence of hemoperitoneum and the absence of free air, there are few radiologic signs in the acute setting that can 
identify a hollow viscus injury. In patients with a significant mechanism and seatbelt sign on physical examination, a high degree of clinical vigilance is required to prevent morbidity from missed bowel injuries, as highlighted by this interesting case.

Integrating an enterprise electronic medical record into trauma resuscitation in the pediatric emergency department. Suzanne Beno', Daniel Rosenfield ${ }^{1}$, Gregory Harvey ${ }^{1,2}$, Karim Fessa ${ }^{1,2}$. From the ${ }^{1}$ The Hospital for Sick Children, Toronto, ON; and the ${ }^{2}$ University of Toronto, Toronto, ON.

Background: As the electronic medical record (EMR) becomes increasingly ubiquitous in emergency departments (EDs) across Canada, acute care scenarios, including trauma resuscitations, require a considered workflow to effectively provide clinical care while contemporaneously recording event data electronically. Team performance, including medication delivery, can be significantly challenged by workflow changes inherent with incorporating an EMR. In a pediatric ED, EMR integration presents both unique challenges and opportunities for success. We describe our experience and lessons learned with this process. Methods: An enterprise EMR (Epic) was incorporated by the hospital. A resuscitation/trauma workflow was designed in alignment with core pediatric safety principles already in effect before EMR adoption, including nonverbal medication dose-specific ordering. Significant safety challenges were encountered during testing and early go-live phases, leading to adoption of a temporary hybrid workflow using both written and electronic functionality, and subsequent workflow refinement in conjunction with the EMR supplier and key hospital stakeholders. Results: Trauma/resuscitation culture in our pediatric institution has traditionally required written orders for enhanced safety, given the challenges of timely dosing of critical medications in children. During initial trauma/ resuscitation EMR adaptation workflow planning, the patient safety benefits of physicians ordering all tests and medications electronically was felt to outweigh potential drawbacks. Full implementation of a planned electronic workflow solution for trauma resuscitation before the EMR go-live date was not possible, and simultaneous trauma activations at the time of go-live demonstrated this initial plan did not adequately meet the needs of the trauma team. Ordering and documenting was not intuitive enough for novice users, many functionalities did not work as intended, the physical size of multiple workstations hampered visualization of the patient, additional personnel were required, and unfamiliarity with the EMR itself compounded confusion. Subsequently, a more deliberate process involving all stakeholders has emerged with the ultimate aim to minimize cognitive load on providers, capture all relevant data efficiently, effectively order and document dose-specific medications, all while assuring computers do not impede team performance. We plan to simulate the new workflows extensively before their implementation, allowing for iterative feedback throughout. This new workflow should deliver a trauma/resuscitation environment that allows for situational awareness of team members while also integrating benefits inherent with use of electronic interfaces (e.g., dose calculation, accurate time stamping). Conclusion: Implementing any EMR is challenging, and using it in critical scenarios, such as trauma, specifically pediatric trauma, is fraught with potential complications. From our experience, extensive user testing and wide stakeholder consultation before go-live is required to assure success. Flexibility with preconsidered contingency plans, such as a hybrid workflow, is vital and may be required. Recognition that EMR adoption timelines may not align strategically with trauma/resuscitation workflow timeline integration is important for planning.

Low-value clinical practices for injury presentations in the emergency department: a scoping review and expert consultation study. Lynne Moore ${ }^{1}$, Pier-Alexandre Tardif ${ }^{1}$, Eric Mercier $^{1}$, Simon Bertbelot ${ }^{1}$, Fiona Lecky ${ }^{2}$, Peter Cameron ${ }^{3}$, Patrick Archambault ${ }^{1}$. From the ${ }^{1}$ Université Laval, Québec, QC; the ${ }^{2}$ University of Manchester, Manchester, England; and ${ }^{3}$ Monash University, Melbourne, Australia.

Background: Injuries led to 3.5 million Canadian emergency department (ED) presentations in 2010 and generated $\$ 4.6$ million in ED medical costs. Tests and treatments that are not supported by evidence and could expose patients to unnecessary harm, referred to here as low-value clinical practices, consume up to $30 \%$ of health care resources. Choosing Wisely has published lists of clinical practices to be avoided. However, few apply to injury, and most are based uniquely on expert consensus. Methods: We conducted a scoping review of the literature followed by an expert consultation survey. We targeted research articles, reviews, recommendations and guidelines that identified at least 1 low-value clinical practice specific to injury care. We documented level of evidence by the number and type of studies reporting on each clinical practice. We then consulted experts in 2 phases: to classify, group and standardize identified ED practices and to rate them according to their potential to be of low value. Results: The systematic scoping review revealed 72226 citations, of which 815 were deemed eligible and led to the identification of 113 ED practices. Of 9 emergency physicians approached to participate in the consultation phase, 8 completed the survey. Following the first phase of consultation, 48 practices were retained. Of these, 33 were considered of clearly or potentially low value by experts, including 5 related to hospital admission for abdominal trauma or mild traumatic brain injury (TBI) and 20 related to imaging, such as computed tomography (CT) or x-ray for mild TBI, ankle, knee, chest and cervical spine injuries. We also identified 15 ED practices in the "grey zone"; i.e., controversial practices not supported by both literature review and expert opinion, including repeat head CT in adults with mild complicated TBI and hospital admission in pediatric isolated skull fracture. Conclusion: This review represents a first step toward developing valid and reliable metrics to monitor lowvalue clinical practices in acute injury care. These metrics will provide a solid basis for the development of interventions targeting de-adoption, such as shared decision-making tools. This study is part of the Canadian Program for Monitoring LowValue Clinical Practices in Injury Care, funded by the Canadian Institutes of Health Research and conducted in collaboration with Choosing Wisely Canada. 
Right patient, right place? Where do seriously injured children receive care in Canada? Alison Macpherson. From York University, Toronto, ON.

Background: Organized trauma systems are associated with significant reductions in morbidity and mortality. Many of Canada's provinces have organized pediatric trauma systems in place. However, the effectiveness of these systems in getting the right patient to the right place has yet to be demonstrated. The objective of this study was to determine the percentage of children with a serious injury diagnosis treated at pediatric trauma centres and to examine the variation in the percentages by province. Methods: Data on children hospitalized for trauma and poisonings across Canada were obtained from the Canadian Institute for Health Information. Injuries were identified as serious if the primary diagnosis was one of the serious injuries. Cross-tabulation identified the percentage of children with these diagnoses seen at pediatric trauma centres, and logistic regression identified the odds of a child with a serious injury going to a pediatric trauma centre. Results: Of the 121741 children hospitalized between 2008 and 2015, 4123 (3.4\%) were identified as having serious injuries. Of these, 2559 (62\%) were seen at pediatric trauma centres. There was considerable variability among provinces, with the percentage of serious injuries treated at pediatric trauma centres ranging from $0 \%$ to $78 \%$. Logistic regression found the odds of being treated at a pediatric trauma centre was 2.1 (95\% confidence interval 1.92.2). Conclusion: Although children with serious injuries are more likely to be treated at pediatric trauma centres, many seriously injured children do not receive care at such centres. This is particularly true in provinces and territories without a pediatric trauma centre. Efforts to improve pathways for seriously injured children may improve these percentages.

Forward aeromedical evacuation team composition: Do different teams impact clinical outcomes? Descriptive systematic review. Colin Laverty ${ }^{1}$, Homer Tien ${ }^{2}$, Andrew Beckett $^{3}$, Avery Nathens ${ }^{4}$, Luis Teodoro Da Luz $^{4}$. From the ${ }^{1}$ Canadian Field Hospital, Gloucester, ON; ${ }^{2}$ Ornge, Mississauga, ON; ${ }^{3}$ Mcgill University, Montreal, QC; and the ${ }^{4}$ Sunnybrook Health Sciences Centre, Toronto, ON.

Background: On the battlefield, forward aeromedical evacuation (forward $\mathrm{AE}$ ) moves patients from the point of injury (POI) to a medical treatment facility (MTF) while providing en-route medical care. Mortality during the POI to MTF phase is extremely significant. Global military forces employ various forward $\mathrm{AE}$ team compositions, each with its own mix of clinicians and interventional capabilities. We conducted a descriptive systematic review on the outcomes of $\mathrm{AE}$ team composition, including mortality. Methods: Medline, Embase and Cochrane databases were searched up to October 2018. Data on aeromedical crew composition and patient outcomes were extracted. Methodological quality was assessed separately for observational (Newcastle-Ottawa) and experimental (Cochrane Collaboration Risk of Bias Tool) studies. The review was conducted in accordance with the Preferred Reporting Items for Systematic Reviews and Meta-Analyses
(PRISMA) guidelines, and strength and quality of evidence was assessed using Grading of Recommendations Assessment, Development and Evaluation (GRADE). Results: We screened 1126 studies. Few were directly relevant to the research question in the military context. The majority of included studies were observational cohorts, mostly retrospective. The quality of cohort studies was low to moderate, as demonstrated by the Newcastle-Ottawa tool and by the GRADE profile assessment. Overall, studies reported a trend for decreased mortality associated with physician presence in forward AE team composition. Conclusion: Data were limited, and further study is required. Nonetheless there was an association between pre-MTF mortality and physician presence in forward $\mathrm{AE}$ team composition. Future research is required to elaborate this trend, eliminate confounders like $\mathrm{AE}$ airframe factors and available medical material, and define an optimal forward $\mathrm{AE}$ team composition in terms of operational effectiveness.

What are the characteristics of field trauma triage "bypass failures" in an integrated, inclusive provincial trauma system? Ian Watson, Susan Benjamin, Allison Chisholm. From the NB Trauma Program, New Brunswick.

Background: The NB Trauma Program introduced field trauma triage (FTT) guidelines to all paramedics in New Brunswick in 2010. Data related to the performance of FTT is captured in the NB Trauma Registry. This study aimed to characterize patients for whom there was evidence of an FTT activation, but who did not bypass a level $\mathrm{V}$ facility despite having documented bypass-qualifying criteria in the prehospital setting. Methods: As part of our regular case review process, nurses working with the NB Trauma Program assess prehospital data and assign quality filters related to FTT at the case level. This allows identification and review of the cases that should have bypassed a level $\mathrm{V}$ centre but did not. We extracted and described the characteristics of these "bypass failure" patients for cases presenting between Apr. 1, 2014, and Mar. 31, 2017, that were subsequently transferred to a higher-level trauma centre. Results: A total of 30 cases were identified during the study period where bypass failure was deemed to have occurred, as compared with 475 cases of FTT activation with hospital bypass occurring as expected (5.9\% bypass failure rate). The cohort of patients who did not bypass a level $\mathrm{V}$ facility despite having bypass-qualifying criteria were $70 \%$ male, had a median age of 53.5 (range 9-98) years, and were most likely to have been involved in a motor vehicle crash $(37 \%)$ or a fall $(30 \%)$. Further, the average length of stay (LOS) in the emergency department (ED) for those who did not bypass a level $\mathrm{V}$ facility despite having bypass-qualifying criteria was 1 hour, 40 minutes, representing a lost opportunity for timely access to definitive care. Conclusion: FTT remains an integral component of the NB Trauma Program. When applied as expected, FTT helps to reduce time to definitive care and LOS in the ED. Users of the FTT guidelines in New Brunswick are encouraged to note the characteristics of patients more likely to result in unexpected bypass failure, allowing educational support to be developed and implemented to help ensure future capture and resulting bypass for similar patient presentations. 
Field trauma triage in New Brunswick: understanding the "immediate life threat" population. Ian Watson, Susan Benjamin, Allison Chisholm. From the NB Trauma Program, New Brunswick.

Background: A provincially standardized field trauma triage guideline (FTTG) was implemented in New Brunswick in 2010. The guideline includes an "immediate life threat" step (step 1A) to ensure those in need of immediate resuscitation are taken to the closest trauma centre. We sought to describe the cohort of patients transported directly to a level $\mathrm{V}$ trauma centre after having been assessed as qualifying under the immediate life threat criteria of the FTTG. Methods: Patients who were assessed by paramedics as having an immediate life threat and who were taken directly to a level V centre between Apr. 1, 2014, and Mar. 31, 2017, were extracted from the NB Trauma Registry. Descriptive methodologies describe predominant mechanisms of injury, length of stay in the emergency department, time to initiate trauma transfer, whether these patients required critical interventions in the level $\mathrm{V}$ centre, and how often each facility received immediate life threat trauma patients. Results: Of the 5381 FTT activations over the study period, 60 (1\%) patients qualified under the inclusion criteria noted above. Over the entire study period, $82 \%$ were found to be males, despite males representing only $50 \%$ of all admitted trauma patients in the NB Trauma Registry over the same period. Motor vehicle collisions accounted for $68 \%$ of these patients, and critical interventions were required in $63 \%$. Oral intubation was the most frequently completed critical intervention ( $47 \%$ of those with any critical intervention), followed by chest tube insertion (20\%). The mean annual interval from arrival at a level $\mathrm{V}$ facility to initiation of the trauma transfer process remained statistically unchanged at 31,38 and 36 minutes, respectively. Five of the province's 12 level $\mathrm{V}$ centres assessed $78 \%$ of the patients overall, with 7 level $\mathrm{V}$ centres seeing fewer than 5 immediate life threat patients during the entire study period. Conclusion: Further exploration of potential sex-based bias in application of FTTG step 1A is indicated. Ongoing educational support is also indicated, as scarce opportunities to apply critical interventions in major trauma is associated with higher risk. Further analysis of patients who did not require any critical interventions may help further refine Step 1A of the FTTG. Finally, despite low enrolment rates, level $\mathrm{V}$ trauma centres appear to consistently activate the process for trauma transfers.

Mild head injury and intentional blasting: Is there a causeeffect relationship? Systematic review of the human, animal and laboratory literature. Colin Laverty ${ }^{1}$, Homer Tien ${ }^{2}$, Andrew Beckett ${ }^{3}$,Avery Natbens, ${ }^{4}$ Luis Teodoro Da Luz ${ }^{5}$. From the ${ }^{1}$ Canadian Field Hospital, Gloucester, ON; ${ }^{2}$ Ornge, Mississauga, ON; ${ }^{3}$ Mcgill University, Montreal, QC; ${ }^{4}$ St. Michaels Hospital, Toronto, ON; and the ${ }^{5}$ Sunnybrook Health Sciences Centre, Toronto, ON.

Background: The relationship between traumatic brain injury (TBI) and exposure to high-energy blasts, such as an improvised explosive device (IED) detonation, has been extensively studied. However, little is known about the relationship between mild TBI and repetitive exposure to intentional low-energy blasts from explosive entry techniques and high-calibre weapon sys- tems. To determine if there is such a cause and effect relationship, we conducted a systematic review of the human, animal, and laboratory evidence. Methods: Medline, Embase and Cochrane databases were searched up to October 2018. Data on outcomes after high-energy blasts were extracted. Methodological quality was assessed separately for observational (NewcastleOttawa) and experimental (Cochrane Collaboration Risk of Bias Tool) studies or a specific tool designed to appraise animal studies. The review was conducted in accordance with the Preferred Reporting Items for Systematic Reviews and Meta-Analyses (PRISMA) guidelines, and strength and quality of evidence was graded using Grading of Recommendations Assessment, Development and Evaluation (GRADE). Results: We screened 2975 studies. There was some evidence of an association between repetitive intentional blast exposure and mild TBI. The limited human and animal data were suggestive of structural and biochemical changes plus neurologic, behavioural, and cognitive symptoms in populations with a repetitive occupational exposure to intentional blasts. The lack of widespread baseline testing in these populations highlights the challenge in establishing a causal relationship. There is also a need to study potential risk mitigation interventions, such as improved personal protective equipment; adapted tactics, techniques, and procedures; preexposure personnel medical screening and baseline testing; markers of disease; and postexposure interventions. Conclusion: Although there is extensive literature on TBI and unintentional blast exposure, there are limited human data on mild TBI and intentional blast exposure. Some laboratory and animal studies augment the limited human evidence. Collectively, the data are suggestive of a cause-effect relationship between mild TBI and intentional blast exposure. Further studies are required to determine acceptable levels of cumulative intentional blast exposure.

Implementation of best practice guidelines on geriatric trauma care: a Canadian perspective. Mélanie Bérubé ${ }^{1}$, Theresa Pasquotti ${ }^{2}$, Barbara Klassen ${ }^{3}$, Angie Brisson ${ }^{4}$, Nancy $T z e^{5}$. From the ${ }^{1}$ Hôpital du Sacré-Coeur de Montréal, Montreal, QC; ${ }^{2}$ Alberta Health Services, Edmonton, AB; ${ }^{3}$ Hamilton Health Sciences, Hamilton, ON; ${ }^{4}$ Vancouver Coastal Health, Vancouver, BC; and the ${ }^{5} \mathrm{McGill}$ University Health Centre, Montreal, QC.

Background: Trauma incidence is consistent with the demographic curve and increasingly affecting the elderly. Considering their reduced physiologic reserves and medical comorbidities, the geriatric trauma population is more likely to experience poorer outcomes than younger patients. The collection of empirical evidence has led to the development of best practice guidelines to optimize the care provided to the geriatric trauma population. The aim of this study was to assess their implementation across Canadian trauma centres. Methods: Using a survey research design, level I to level III trauma centres were approached to complete the survey in the fall of 2017. The survey questionnaire included 14 questions aimed at establishing the profile of surveyed trauma centres and the level of implementation of the Geriatric Trauma Management Guidelines of the American College of Surgeons - Trauma Quality Improvement Program. Trauma program coordinators or managers completed the survey. Descriptive statistics were computed for data analysis. Results: Fifty-one trauma centres completed the survey, among which $30 \%$ were 
level I, 42\% level II and 28\% level III. Data were obtained from 8 provinces with the following response rates: British Columbia (11/11), Alberta (9/9), Saskatchewan (2/2), Ontario (8/9), Quebec (15/28), New Brunswick (4/8), Nova Scotia (1/1) and Prince Edward Island (1/1). The mean percentage of elderly patients ( $\geq 65 \mathrm{yr}$ ) with a severe injury (Injury Severity Score $\geq 12$ ) was nearly $30 \%$. The most frequently used cut-off age to define a geriatric trauma was $\geq 65$ years $(36 \%)$. Close to half ( $46 \%$ ) of the trauma centres did not use a cut-off age, and among these $20 \%$ identified the presence of a geriatric trauma based on patient frailty. Less than a quarter (24\%) of centres acknowledged employing trauma team activation criteria specific to the geriatric population. Specialized geriatric trauma resources were involved in patient care in $36 \%$ of trauma centres. Implementation rates of commonly evaluated geriatric issues were dysphagia (68\%), fall risk $(64 \%)$, delirium (62\%), malnutrition (58\%), substance abuse (42\%), identification of senior at risk $(40 \%)$, functional status (40\%), depression (38\%) and frailty (28\%). Protocols for care optimization were applied as follows: anticoagulant reversal $(56 \%)$, early mobilization $(56 \%)$, transition of care $(54 \%)$, advanced directives within 72 hours (52\%), geriatric pain management orders (32\%), Beers criteria to adjust medication (10\%), and inpatient geriatric trauma orders (8\%). Conclusion: A growing number of elderly trauma patients with severe injury are admitted in Canadian trauma centres annually. Despite this, few centres use specialized geriatric trauma resources. Moreover, more effort is required to promote the uptake of best practice guideline recommendations in geriatric trauma care across Canada.

Alberta prehospital trauma system survey - phase 1. Shaun Cowan ${ }^{1}$, Vanessa Fawcett ${ }^{1}$, Bonnie Tsang ${ }^{1}$, Alison Kabaroff ${ }^{1}$ Kevin Verboeff ${ }^{1}$, Simon Turner ${ }^{1}$, Michael Kim ${ }^{1}$, Sandy Widder ${ }^{2}$. From the ${ }^{1}$ University of Alberta, Edmonton, $\mathrm{AB}$; and the ${ }^{2}$ University of Alberta Hospital, Edmonton, $\mathrm{AB}$.

Background: Trauma systems are complex and involve many multidisciplinary providers and teams. The Alberta trauma system is unique in its geography and organization and in that it is governed by a single health care authority. To better understand the barriers and challenges in providing quality trauma care in the province, a study was conducted to better explore areas of improvement within the prehospital care environment. Methods: This qualitative, constructivist thematic analysis study seeks to uncover the challenges and successes perceived by front-line staff and operational leadership who are prehospital care providers within the Alberta trauma system through a 3-phase approach. Phase 1 consists of individual interviews with prehospital leadership in communications, operations, education and air transport. Phases 2 and 3 will survey front-line providers through an online survey and focused interviews, exploring and comparing themes from phase 1 and additionally identified themes. Results: Phase 1 raw data consist of recorded, individual interviews of 12 leaders in Edmonton and North zones. These zones were selected to include a rural and metropolitan area of the province. Transcripts of the interviews were coded and analyzed for themes based on semistructured questions in a 60-minute interview per participant and collated for repeating areas or themes. Themes of strength that were identified included a robust standardized communication network across the province, including transport and medical director physician support; air transport coordination; and automated triggers for trauma care resources within the dispatch system. Additionally, trauma destination bypass process for rural crews was perceived to be an improvement in trauma care. Areas of perceived challenge included understanding trauma team activation criteria, clear direction regarding the rationale for prenotification of trauma centres, and a system of mass casualty event management which differed from day-to-day operations. Understanding the trauma team composition at the trauma centre teams was broadly present, but a lack of understanding of the deliberate response by trauma teams was common. Overall, there was a strong desire to improve on the quality of prehospital care provided, including a better understanding of roles and responsibilities of trauma team members, collaborative interdisciplinary educational rounds, and case/patient follow-ups. Conclusion: Phase 1 of our project has identified several areas of strength and challenge, with themes centred around multidisciplinary collaboration and education, patient outcome feedback, and understanding other aspects of the trauma system. These findings will direct future goals to collaborate and improve trauma care among the prehospital and in-hospital providers in the Alberta trauma system as well as inform other organizations in striving toward trauma care excellence.

The role of diagnostic imaging in the initial evaluation of blunt abdominal trauma: a comparison of international guidelines. Kai Homer ${ }^{1}$, Christopher Fung ${ }^{1}$, Sandy Widder ${ }^{2}$, Michael Kim ${ }^{1}$. From the ${ }^{1}$ University of Alberta, Edmonton, $\mathrm{AB}$; and the ${ }^{2}$ University of Alberta Hospital, Edmonton, AB.

Background: There is substantial variation among practitioners and institutions in the choice of imaging obtained for the evaluation of blunt abdominal trauma (BAT). There are several existing guidelines that contain evidence-based recommendations for imaging investigations in BAT published by different clinical specialties. Our purpose was to assess whether there are discrepancies between recommendations for BAT imaging according to specialty (emergency medicine v. trauma v. radiology) or country of origin. Methods: Following their retrieval via online search, recommendations from 7 documents published between 2002 and 2017 by the following societies were compared: American College of Radiology, Eastern Association for the Surgery of Trauma, National Institute for Health Care and Excellence, Government of Western Australia, American College of Emergency Physicians, American College of Surgeons, and Task Force for Advanced Bleeding Care in Trauma. The primary literature reviewed in these documents was published between 1987 and 2016. Results: Statements reflecting areas of unanimous agreement among guidelines are as follows: 1) prehospital ultrasound does not affect clinical outcomes; 2) hemodynamically unstable patients with clear clinical evidence of intraabdominal injury (IAI) should proceed to laparotomy without imaging; 3) radiographs have a role in the primary survey of hemodynamically unstable BAT patients; 4) plain radiographs should not be obtained in stable patients who may safely access more advanced imaging; 5) the focused assessment with sonography in trauma (FAST) exam should be performed for the purpose of identifying hemodynamically unstable BAT patients who should immediately proceed to laparotomy; 6) FAST has a limited role in the evaluation of stable BAT; 7) contrast-enhanced 
computed tomography (CT) is the study of choice in hemodynamically stable BAT patients; and 8) patients with hematuria should be evaluated for renal injury with contrast-enhanced CT scan (with 2 documents further specifying CT urography). Statements reflecting areas of disagreement among guidelines are as follows: 1) oral contrast at CT may enhance diagnostic accuracy for pancreatic or duodenal injury; and 2) in conjunction with clinical judgment, negative CT scan is adequate to discharge hemodynamically stable BAT patients. Conclusion: There is significant agreement among recommendations regarding early imaging for the evaluation of BAT patients, particularly the initial use of FAST for unstable patients and priority of contrastenhanced CT for stable patients. The few minor disagreements among guidelines that were identified are not associated with either the geographic origin or the specialty of the authors. Existing variations in the specific investigations obtained in clinical practice cannot be accounted for by variation in recommendations in published guidelines.

Comparison of injury care structures, processes and outcomes in integrated trauma systems in Quebec, Canada, and the United Kingdom. Samy Bouderba ${ }^{1}$, Lynne Moore ${ }^{1}$, Fiona Lecky $^{2}$, Tom Lawrence ${ }^{2}$, Kabina Soltana ${ }^{3}$, Thowiba Mansour ${ }^{1}$. From ${ }^{1}$ Université Laval, Québec, QC; the ${ }^{2}$ University of Manchester, Manchester, UK; and the ${ }^{3} \mathrm{CHU}$ de QuébecUniversité Laval Research Centre, Québec, QC

Background: Identifying unwarranted variation between hospitals in injury processes and outcomes is the key to identifying areas for quality improvement. Risk-adjusted comparisons across trauma systems may be even more informative. Health systems in the UK and Quebec are based on single government payer health care and both have inclusive, integrated trauma systems. Our objectives are to compare trauma system structures, processes of care and patient outcomes between integrated trauma systems in the UK and Quebec, Canada. Methods: We conducted a multicentre, retrospective cohort study based on the integrated trauma system of Quebec, Canada, and the National System of Regional Trauma Networks of England \& Wales between 2015 and 2017. All patients admitted for major trauma (Injury Severity Score > 12) were included. Trauma systems were compared using trauma system elements frameworks recommended by the World Health Organization and the American College of Surgeons Committee on Trauma. Results: The study sample comprised 70342 cases from the UK and 9010 cases from Quebec. Mean age and proportion of men were similar in the 2 systems (60 $\mathrm{yr}$ and 68\%). More patients were admitted following low falls in the UK $(45 \% \mathrm{v}$. $23 \%$ ) and more were admitted following motor vehicle collisions in Quebec (33\% v. 28\%). Head trauma was more common in the UK (58\% v. $47 \%)$, whereas more spinal cord injuries were reported in Quebec (11\% v. 6\%). Admission to the intensive care unit (ICU) was more common in Quebec (40\% v. 28\%). Crude mean hospital length of stay was longer in UK than Quebec $(17.2 \mathrm{~d} \mathrm{v} .15 .6 \mathrm{~d})$, and the same was observed for ICU stay $(8.1 \mathrm{~d}$ v. 6.7 d). Crude mortality was $13 \%$ in Quebec and $11 \%$ in the UK. Conclusion: This study provides information on differences in trauma system structure, adherence to best practice clinical processes and patient outcomes, including mortality, morbidity and resource use between 2 single government payer health care systems with integrated trauma systems. Information may be used to inform quality-improvement initiatives in both countries. The next step will be to conduct risk-adjusted and subgroup comparisons.

Adherence to guidelines on low-value clinical practices in acute trauma care. Kabina Soltana, Lynne Moore, Samy Bouderba, Alexis Turgeon, Radoslav Krouchev, Eric Mercier. From the CHU de Québec-Université Laval Research Centre, Québec, QC.

Background: Inappropriate or unnecessary tests and procedures have been identified as one of the most important areas of excess health care spending. Internationally recognized injury care organizations produce guidelines that include recommendations on clinical practices that should be avoided. However, we lack data on how these recommendations are applied in practice. The aims of this study are to identify low-value practices in injury care guidelines and evaluate how frequently they are used in practice. Methods: We identified low-value clinical practices from internationally recognized injury organization guidelines. We then developed algorithms to measure the frequency of these practices using trauma registry data and validated them with clinical experts. Finally, we applied the algorithms using data from an integrated Quebec trauma system and calculated frequencies. Results: We used the latest recommendations from 4 internationally recognized professional organizations: Eastern Association for Trauma, American College of Surgeons, Brain Trauma Foundation and American Trauma Society. After expert consultation, 14 practices were retained. We developed and validated algorithms for 9 of these practices. Examples include therapeutic hypothermia in adults with traumatic brain injury and intracranial pressure responding to other stage 2 treatments $(0.32 \%)$, chest $\mathrm{x}$-ray in hemodynamically stable patients with blunt chest trauma and a normal physical exam (10.8\%), and laparotomy in hemodynamically stable patients with blunt liver or spleen injuries $(2.2 \%$ and $4.5 \%$, respectively). Conclusion: The results of this study advance knowledge on low-value practices in acute injury care. Results suggest that adherence to guidelines on low-value care can be evaluated using trauma registry data. In general, the frequency of low-value care in our trauma system was low.

Is the thrill worth it? A road safety program for teens. Liane Fransblow, Debbie Friedman, Angeliki Souranis, Lawrence Slapcoff. From the Montreal Children's Hospital, Montreal, QC.

Background: In Canada and in Quebec, the leading cause of death due to unintentional injury in teenagers is motor vehicle collisions. The Montreal Children's Hospital created an educational program targeting high school students. "Is the thrill worth it?" includes a presentation and a peer-led road safety campaign targeting students in grades 10 and 11 . The goal of this program is to improve knowledge of the risks associated with driving among high school students. Methods: Satisfaction questionnaires were provided to students who received the presentation to determine if they felt it was useful and engaging. Student leaders also filled out a monitoring questionnaire about the "student leadership in injury prevention program" (SLIPP). Results: In total, 174 students in 3 schools answered the presentation questionnaires, and 5 student leaders from 1 school answered the 
SLIPP leader questionnaire. Most students who received the presentation felt it was relevant, that it enhanced their knowledge on the topic of road safety and would recommend it to grade 10 and 11 students. The sections that they reported were most impactful were a video testimonial and statistics related to young drivers. The SLIPP leaders reported that they had adequate support from both their school and the hospital representative to implement their activities, and they felt that their activities led to an increase in awareness of road safety topics among their peers. Conclusion: Both students who received the presentation and those who were SLIPP leaders were highly satisfied with the "Is the thrill worth it?" program. The combination of a peer-led program targeting high school students with a presentation offered by a trauma centre appears to be an effective method to reach high school students on the topic of road safety.

Training health professionals on car seat safety. Liane Fransblow $^{1}$, Debbie Friedman ${ }^{1}$, Marouan Bel Fakir ${ }^{2}$. From the ${ }^{1}$ Montreal Children's Hospital, Montreal, QC; and the 2Societe de l'assurance automobile du Quebec, Gatineau, QC.

Background: Car seat misuse in Quebec and across the country is prominent. When parents receive prevention information from a health professional, they are likely to perceive it as relevant and important. As a pediatric trauma centre attached to a birthing centre, we trained nurses and other health care professionals on proper car seat use. Our aim is to improve knowledge of car seat use and confidence in verifying infants in car seats among health professionals. Methods: Nurses and other health professionals at the Montreal Children's Hospital and the Birthing Centre at the Royal Victoria Hospital were provided a brief training session on the proper use of car seats in infants and children by the Société d'Assurance Automobile du Quebec. Staff were asked to complete a pre/post questionnaire to evaluate the effectiveness of this training. Results: Forty-eight participants answered the questionnaires. Most (74\%) participants were nurses, followed by occupational therapists, audiologists and social workers. Most nurses worked in the maternity, neonatal intensive care unit and pediatric medicine departments. Prior to the training session, less than one-third of the participants reported that they were comfortable discussing car seat safety with their patients. This increased to $100 \%$ of participants following the brief training session. All participants reported that they felt the training was useful. Increased knowledge was shown across all variables. Additionally, staff commented that they appreciated the training and would recommend the training to their colleagues. Conclusion: Infants often arrive at hospitals in a bucket car seat. This can be a perfect moment to discuss car seat safety. A brief training session on the proper use of car seats can improve knowledge of health professionals. Health professionals are given the tools necessary to verify quickly if the patient is adequately secured in the seat and to explain to parents how to properly buckle their child into a car seat.

Validation of an adaptation of the standardized swallowing assessment tool in patients with moderate-severe traumatic brain injury and cervical spine cord injury. Mélanie Bérubé $^{1}$, Valérie Turcotte ${ }^{1}$, Marie-Pierre Valiquette ${ }^{1}$, Françis Bernard ${ }^{1}$,Marc Giroux', Marie-Ève Côté ${ }^{1}$, Annik Gagné ${ }^{1}$, Stéphanie Dollé ${ }^{1}$ Céline Gélinas ${ }^{2}$. From the ${ }^{1}$ Hôpital du
Sacré-Coeur de Montréal, Montreal, QC; and ${ }^{2} \mathrm{McGill}$ University, Montreal, QC.

Background: Dysphagia is a frequent consequence of moderatesevere traumatic brain injury (TBI) and cervical spinal cord injury (SCI), which may lead to malnutrition and aspiration pneumonia, potentially increasing patient morbidity and mortality. Although best practice trauma guidelines recommend early screening for dysphagia, there is no validated tool for use in this population. This study aimed to assess the validity and reliability of an adaptation of the Standardized Swallowing Assessment (SSA) tool specific to these trauma patients. Methods: Following a French translation and the addition of 2 items to the SSA, a repeatedmeasures design was used to assess inter-rater reliability and criterion validation. Also, content validation and satisfaction with its use were evaluated. Sixty patients ( 27 with TBI and 33 with SCI) and 18 health care professionals assessed the SSA reliability and validity. Twenty-five critical care nurses who administered the adapted SSA to targeted patients on at least 3 occasions assessed satisfaction. Results: Diagnoses of study participants were moderate TBI (33\%), severe TBI (17\%), SCI American Spinal Injury Association (ASIA)-A (7\%), SCI ASIA-B (4\%), SCI ASIA-C $(23 \%)$ and SCI ASIA-D (13\%). Mean age was $57 \pm 21$ years and mean duration of intubation was $56 \pm 94$ hours. The $\kappa$ coefficient between SSA raters was 0.80 when considering all participants, with 0.85 for TBI, and 0.75 for SCI participants. The content validity index (CVI) for the SSA items varied from 0.82 to 1.00 , while the CVI was 0.96 for all scale items. The item: "Is the patient able to lick top and bottom lip?" obtained the lowest CVI. Sensitivity was $91 \%$ and specificity was $45 \%$ when comparing dysphagia screening by nurses to the speech pathologist evaluations (gold standard criterion). The area under the receiver operating characteristic curve was 0.74 (95\% confidence interval $0.50-0.98)$. Nurses' satisfaction rates with the SSA were $72 \%-$ $96 \%$ for the tool appropriateness, $88 \%-96 \%$ for the accessibility of the materials, $59 \%-95 \%$ for the required processes and $42 \%-$ 96\% for its acceptability. Lower satisfaction scores (< 70\%) were as follows: 1) "I believe that patients appreciate being screened for dysphagia" (42\%), and 2) "I face obstacles when administering the SSA" (59\%). A large proportion $(25 \%-50 \%)$ of nurses provided neutral responses to these items. Conclusion: Study findings supported the reliability and content validation of the adapted SSA. High sensitivity was demonstrated, and the tool showed good capacity to discriminate for the presence and absence of dysphagia. However, specificity was low. Although the small number of nondysphagic participants could explain this finding, nurses should ask for a more comprehensive evaluation when dysphagia screening is uncertain. Finally, the use of the adapted SSA was associated with a high level of satisfaction.

Profile and performance of the Quebec adult trauma system 2013-2016. Catberine Gontbier ${ }^{1}$, Amina Belcaïd ${ }^{2}$, Catberine Truchon ${ }^{1}$, Lynne Moore ${ }^{3}$, Julien Clément ${ }^{2}$, LouisPhilippe Pelletier'. From the ${ }^{1}$ Institut national d'excellence en santé et en services sociaux, Montreal, QC; the ${ }^{2} \mathrm{CHU}$ de Québec-Université Laval, Québec, QC; ${ }^{3}$ Université Laval, Québec, QC; and ${ }^{4}$ CISSS Laurentides, Québec, QC.

Background: The Institut national d'excellence en santé et en services sociaux (INESSS) has the mandate to evaluate, on a 
continuous basis, the Quebec trauma system, both at health care facilities and at more system-wide levels. The objective of this study is to describe the profile of adult patients, as well as the performance of the system on key process and results indicators between 2013 and 2016. Methods: Using the Quebec Trauma Registry, we performed a retrospective cohort study of patients older than 16 years admitted for traumatic injury between 2013 and 2016 in 1 of the 57 adult trauma centres. Descriptive data on patient profile, episodes of care and trajectories within the network were generated. A global mean for the province as well as a distribution plot of the 57 facilities, by level of care, was computed for each performance indicator. Results: Over the 3-year period, a total of 52190 adult patients were admitted in a trauma centre, with major cases (Injury Severity Score $\geq 12$ ) accounting for $19 \%$ of all admissions. Nearly $80 \%$ of all patients were transported by ambulance directly to definitive care, with a mean delay of 1 hour, 53 minutes, but with important inter-region variations. Minor and major orthopedic injuries were the most commonly diagnosed injuries in the system, with $44.2 \%$ and $17.5 \%$ of all cases, respectively. Mean age for men and women were 55.7 years and 70.6 years, respectively. At discharge, most patients were going home $(43.3 \%)$, and had external follow-up (18\%) or rehabilitation services $(9 \%)$. Thirteen process performance indicators were analyzed, with an overall conformity varying from $12 \%$ to $95.4 \%$. Processes with standardized clinical protocols (e.g., antibiotic prophylaxis) showed the highest level of performance, whereas processes dependent on resource availability (e.g., surgical delays) showed the lowest results. Risk-adjusted mortality for all patients was $6.4 \%$, and $18.7 \%$ of trauma patients had at least 1 complication during their hospital stay. Patients aged 65 years and older had more unplanned readmissions $(10.2 \%)$ and longer mean length of stay (12.1 d). Conclusion: This global analysis allowed identifying priorities for improvement in terms of prevention, strengthening of inter-facility transfers, and standardization of certain clinical processes. These results will also inform a revision of the content as well as the format of the facilitylevel performance reporting mechanisms.

The burden of penetrating traum in British Columbia from 2010 to 2015. Anthony Bryson ${ }^{1}$, Vesna Ivkov², Karli Gamble ${ }^{1}$, Lisa Constable ${ }^{1}$, Foe Haegert ${ }^{1}$. From the ${ }^{1}$ Royal Columbian Hospital, New Westminster, BC; and the ${ }^{2}$ Fraser Health Trauma Network, Vancouver, BC.

Background: Penetrating trauma causes a significant burden to Canadian health care systems. Despite the management of penetrating trauma being of high priority for trauma systems, the epidemiology of these injuries has not been fully elucidated in British Columbia (BC) in recent literature. The purpose of this study is to investigate the demographics, types, and motivations of penetrating trauma in BC from Jan. 1, 2010, to June 30, 2015. Methods: This is a retrospective cohort study using routinely collected data retrieved from the BC Trauma Registry (BCTR) and the Discharge Abstract Database. The databases include demographic information, the modality of injury, motivation, and disposition of the patients. All patients with a penetrating trauma (firearm, knife, or sharp object) and Injury Severity Score $>9$ who were admitted or transferred to a BCTR facility were included in the study. Results: The BCTR and DAD captured 168025 discrete traumatic patient encounters during the study period. Of those, 4740
(2.8\%) were coded for penetrating trauma and included in the analysis. Most (72.8\%) patients were male and between age 25 and 64 years $(65.8 \%)$. Most penetrating trauma presenting to the emergency departments were from knives or other forms of stab wounds $(93.0 \%)$, and had an unknown motivation (57.1\%). The highest incidence of penetrating trauma was in the Fraser Health Authority (31.3\%), followed by the Vancouver Coastal Health Authority (19.9\%), Interior Health Authority (18.7\%), Vancouver Island Health Authority (18.0\%), and the Northern Health Authority (12.1\%). Most patients were discharged home without supports $(87.1 \%)$, and $1.0 \%$ died from their injuries. Conclusion: This analysis informs the demographics and types of penetrating trauma in British Columbia, while incomplete data limit analysis of motivation. The burden of penetrating trauma is likely underreported in this study owing to patients who died before arrival at a BCTR facility not being included in the databases.

Mortality of the "stable" abdominal trauma patient: a race against time. Faran Bokbari, Francesco Bajani, Chib-Yuan Fu, Stanley Welsh, Matt Kaminsky, Andrew Dennis, Fredric Starr, Thomas Messer, Caroline Butler, Leab Tatebe, Statbis Poulakidas. From the John H. Stroger Hospital of Cook County, Chicago, IL.

Background: Surgical treatment is usually required in the management of penetrating abdominal injury. Delayed operation may result in increased morbidity and mortality in unstable patients. Factors that increase this morbidity and mortality, specifically in stable patients, are not well defined. We looked at the characteristics of stable and unstable patients undergoing operative intervention for penetrating abdominal trauma. Methods: The National Trauma Data Bank (NTDB) was queried for penetrating abdominal trauma patients from 2007 to 2015 . The time to abdominal operation was evaluated both in stable and unstable patients. Multivariate analyses were carried out to identify factors independently associated with mortality among patients with penetrating abdominal trauma. Results: A total of 31662 penetrating abdominal trauma patients who received abdominal operations were evaluated. Of these, $20.2 \%$ underwent surgery 2 hours after arrival at the emergency department (ED). Among unstable patients (systolic blood pressure $[\mathrm{SBP}]<90 \mathrm{~mm} \mathrm{Hg}$ ), those who received abdominal surgeries within 2 hours, deceased patients had significantly longer ED time than survivors $(30.9 \mathrm{~h}, p<0.001)$. Mortality increased $0.9 \%$ for each additional minute in the ED for unstable patients. For stable patients (SBP $\geq 90 \mathrm{~mm} \mathrm{Hg}$ ) who received an abdominal operation 2 hours after ED arrival, the transportation time, ED time and time to operation were not related to mortality. In these patients, mortality was independently associated with age, Injury Severity Score, SBP, Glasgow Coma Scale score on arrival, and the presence of gastrointestinal tract injury. Conclusion: In penetrating abdominal trauma, while unstable patients require prompt surgical intervention, seemingly stable patients have an increased mortality that can be predicted by presenting patient characteristics.

The challenges in interpreting the TQIP definitions of complications and comorbidities. Alma Rados. From Alberta Health Services, Calgary, AB.

Background: Foothills Medical Centre (FMC) started submitting to theTrauma Quality Improvement Program (TQIP) in 2015. 
Since then, numerous challenges in terms of documentation and terminology have been identified by the data analysts. These challenges included complications and comorbidities not being documented as per the TQIP standards by the admitting services. There was uncertainty among the data analysts regarding some of the definitions of comorbidities and complications. This was the rationale for selecting comorbidities and complications to be assessed during interrater reliability. Methods: Interrater reliability is performed at FMC quarterly. From these assessments, it was noticed that there were discrepancies between the data analysts. These discrepancies included inconsistent interpretation of comorbidities and complications. After receiving clarification on the definitions of the complications and comorbidities from TQIP, interrater reliability was reassessed among the data analysts. Results: The interpretation of the definitions for the complications and comorbidities were more consistent among the data analysts when the interrater reliability was reassessed. There are still challenges with the admitting services not documenting as per the TQIP standards. However, the data analysts are clearer and more confident to determine which comorbidities and complications can be abstracted based on TQIP's definitions. Conclusion: The TQIP definitions for comorbidities and complications are specific, requiring the data analysts to thoroughly understand the rationales for the definitions. This will help to ensure that the complications and comorbidities are abstracted accurately and consistently. The data analysts continue to further their education and understanding of the TQIP definitions by attending educational sessions, completing monthly quizzes, and reaching out to TQIP when further clarification is needed.

A Quebec trauma centre experience in implantation of costal fixation for severe thoracic trauma: the impact on physical and psychological function after surgery. Valérie Courval, Daniel Thauvette. From the Université Laval, Québec, QC.

Background: Costal fixation for thoracic trauma seems to help the recovery of patient. However, the literature still shows controversy for the indication of surgery. This study is a retrospective review of our first cases of patients selected with strict radiologic and clinic criteria. The objective of this study was to demonstrate that this procedure is safe, with low morbidity and mortality, and can help for the recovery of patients selected with strict surgical indication. Methods: We reviewed the charts of the 19 patients who had a costal fracture fixation since 2015. We looked at the patient characteristics, the trauma information, the surgical information and the hospitalization data. We did a follow-up of the patients with a formal survey to evaluate their physical and psychological evolution in the postoperative course a few months after surgery. Results: Nineteen patients had surgery for costal fracture fixation. Four patients had a blunt thoracic trauma, 14 had a blunt polytrauma and 1 patient had an hold trauma and was fixed for a massive transdiaphragmatic hernia and pulmonary hernia secondary to costal fracture. The indications for the surgical procedure were respiratory insufficiency $(n=3)$, difficult ventilatory weaning $(n=5)$, flail chest and thoracic instability $(n=10)$, residual pain $(n=2)$ and hemodynamic instability $(n=1)$. The surgery occurred 7,5 (range 2-198) days after the accident. The median number of costal fractures per patient was 11, with a median of 4 fixed by surgery. One patient needed a bilateral fixation. Postoperative complications were pneumonia, pleural effu- sion, pneumothorax, and hematoma. For the 11 patients who needed mechanical ventilation, the median time for ventilation weaning after surgery was 9 days. Ten patients completed the 36-item Short Form Survey Instrument (SF-36) in the follow-up after surgery. The score at the SF-36 for the physical outcome was $59 / 100$ and the psychological outcome was 65/100. Two patients died after surgery, but not from a complication due to the surgery. One died from respiratory insufficiency and the family decided to end treatment, and the other patient died a few months after the surgery from a cause not known yet. Conclusion: In this tertiary trauma centre, the patients were selected with strict criteria, and only those with severe thoracic trauma underwent costal fracture fixation. The follow-up in the Quebec trauma centre showed that in this cohort of complex thoracic trauma patients, physical and emotional function was preserved and the SF-36 results were similar to the results of the average Canadian population $(50-55$ on physical and psychological results).

Application of Lean methodology to optimize trauma registry abstraction. Cathy Masales ${ }^{1}$, Paul Engels ${ }^{2}$, Barbara Klassen ${ }^{1}$, Angela Coates ${ }^{3}$, Sudarshi De Silva ${ }^{1}$. From ${ }^{1}$ Hamilton Health Sciences, Hamilton, ON; ${ }^{2}$ McMaster University, Hamilton, ON; and the ${ }^{3}$ Hamilton Health Sciences Trauma Program, Hamilton, $\mathrm{ON}$.

Background: Our level I trauma centre contributes approximately 1000 submissions to the Ontario Trauma Registry (OTR) annually. As a new participant of the Trauma Quality Improvement Program (TQIP), we observed increasing pressures to meet monthly data submission and reporting expectations. In order to maximize our productivity, we adopted a Lean performance improvement method to uncover problems in our workflow process and efficiency. We identified differences in the time taken by each abstractor to close their assigned task. Methods: There are many Lean tools available for use to improve a process. We selected the A3 report as our tool. In its basic form, the A3 template is divided into quadrants to guide problem solving: 1) establish the problem statement; 2) describe the current state of the problem, including a cause analysis; 3 ) identify the target state; and 4) prescribe an action plan for getting it done, including a follow-up process. Results: Abstractor delays in data "closing" resulted in a time crunch to prepare data submissions and reports. The metrics focus on how long it takes for work (i.e., trauma registry cases) to flow through our system. Individual abstractor metrics were calculated to highlight differences in individual processes and not for suboptimizing individual performance. Current state showed no differences in the complexity of cases defined by mean length of stay (LOS) and mean Injury Severity Score (ISS) of abstractor case volumes (mean LOS $10.15 \mathrm{~d}$ v. $10.93 \mathrm{~d}, p=$ 0.644 ; mean ISS 16.51 v. $15.28, p=0.060$ ). Analysis of our flow metrics between abstractors identified significant differences between abstractors for the cycle time from patient discharge to the date that the record was closed in the registry $(p<0.001$ for LOS categories). Times from discharge to case closure were consistently shorter for Abstractor A (34.0 d v. 50.3 d), with greater standard deviations (SDs; 17 v. 12). Greater SD was interpreted as providing greater flexibility in the case closure process. Using Lean methodology, counter measures were developed to reduce over-processing waste and improve data flow: 1) identification and prioritization of source documents, 2) creation of a "Daily 
Case Closure Log" and 3) feedback of individual abstractor "work in process" metric. After 3 months, we observed greater standardization in case closure practice and no significant differences the cycle time metrics. Conclusion: Using the Lean performance improvement methodology and implementing counter measures, we were able to improve productivity and minimize pressures to meet monthly data submission and reporting expectations. Standardizing processes has minimized the differences in the time taken for each abstractor to close their assigned workload.

Prehospital vital signs accurately predict initial emergency department vital signs. Marc D. Trust, Morgan Schellenberg, Subarna Biswas, Kenji Inaba, Vincent Cheng, Zachary Warriner, Bryan Love, Demetrios Demetriades. From the LAC+USC Medical Center, Los Angeles, CA.

Background: Prehospital vital signs are often used to triage trauma patients and assist with resource allocation. Concerns exist regarding the accuracy of vital signs taken in the field, and correlation between prehospital and initial emergency department (ED) vital signs has not been studied on a large scale. The goal of the study is to assess correlation of prehospital and initial ED vital signs among patients meeting American College of Surgeons Committee on Trauma criteria for trauma team activation. Methods: Patients presenting to our level I trauma centre (2008-2018) were included if they triggered prehospital trauma team activation. Patients with unrecorded vital signs were excluded. Demographics, injury data, and prehospital and initial ED vital signs (systolic blood pressure $[\mathrm{SBP}]$, pulse pressure $[\mathrm{PP}]$, heart rate $[\mathrm{HR}]$, respiratory rate $[\mathrm{RR}]$, and Glasgow Coma Scale [GCS] score) were abstracted. Prehospital and initial ED vital signs were compared using Bland-Altman intraclass correlations (good agreement, > 0.60; fair, 0.40-0.60; poor, < 0.40). Results: After exclusions, 15321 patients were included in the study. Median age was 34 (interquartile range [IQR] 23-52) years, and 11622 patients (76\%) were male. Mechanism of injury was blunt in 12042 (79\%). Median transport time was 15 (IQR 11-21) minutes. The mortality rate was 3\% $(n=513)$. Mean changes in vital signs between the field and ED were $-0.024 \mathrm{~mm} \mathrm{Hg}$ in SBP, $+0.027 \mathrm{~mm} \mathrm{Hg}$ for $\mathrm{PP},+0.051$ beats $/ \mathrm{min}$ in HR, -0.01 breaths per minute in RR, and -0.00 points in GCS. Prehospital and initial ED vital signs demonstrated a good agreement for GCS (intraclass correlation $0.79,95 \%$ confidence interval [CI] 0.77-0.79), a fair agreement for SBP $(0.48,95 \%$ CI $0.46-0.49)$ and $\operatorname{HR}(0.59,95 \%$ CI $0.56-0.61)$, and a poor agreement for PP (0.32, 95\% CI $0.30-0.33)$ and RR (0.13, 95\% CI 0.11-0.15). Conclusion: Despite inherent difficulties with prehospital assessments, prehospital GCS, SBP and HR correlate well with initial ED vital signs in an urban trauma setting with short transport times. PP and RR are less reliable, potentially due to patient decompensation or to challenges in obtaining accurate field measurements. Additional studies are needed to elucidate these differences further. At present, prehospital GCS, SBP, and HR can be considered accurate predictors of initial ED vital signs.

Trauma registry implementation in a western Kenya referral centre: highlighting the quality improvement approach. Fessica Hogan. From the University of Alberta, Edmonton, AB.

Background: Injury is a leading cause of morbidity and mortality in low- and middle-income countries like Kenya. Exact causes of trauma have remained unclear owing to inconsistent data collection throughout the spectrum of patient care. Trauma registries are used to better appreciate injury and support effective public policy decision-making. Leveraging quality-improvement science, a trauma registry was created and implemented with partners at the Moi Teaching \& Referral Hospital, the second largest government hospital in Kenya. Methods: The Donabedian health services model, along with the WHO Guidelines for Essential Trauma Care and Australasian Trauma Verification Program, were used to understand the referral centre's capacity and to perform a 6-month chart audit using a trauma registry data form. A root-cause analysis was created to implement the trauma registry while plan, do, study, act rapid change cycles were applied to improve and compare the quality of injury data collected prospectively over 6 months. Results: Facility assessments, fishbone diagrams, and pareto charts on key challenges around trauma data collection were created. A trauma data collection form was refined to suit the hospital site's needs and used to collect data on all 354 trauma files that could be found in medical records from June 2016 to December 2016. Between September 2017 and March 2018, 1918 trauma forms were filled for patients presenting to the Moi Teaching and Referral Hospital for care of traumatic injury, capturing nearly 5.5 times more trauma patients than the previous system over a comparative time frame. While not all data were collected more frequently, such as occupation of the patient (not recorded in $3 \%[n=12]$ retrospectively and $23 \%$ $[n=446]$ prospectively), vital public health policy-specific data showed significantly higher capture rates. County location of injury was recorded retrospectively only $49 \%(n=36)$ of the time, while more than $99 \%(n=1899)$ were collected prospectively. The chart audit also showed that helmet use for cyclists and motorcyclists was recorded in only $15 \%$ of applicable charts, but was recorded in $43 \%$ of prospective trauma files. Outpatients also appeared to be captured more frequently in the pilot study, as $3 \%$ $(n=11)$ of retrospective trauma files were outpatients and $28 \%$ $(n=541)$ of prospective charts were those of outpatients. Conclusion: Implementing a sustainable trauma registry requires meaningful collaboration with local interdisciplinary teams, which proved to be critical in anticipating challenges unique to the Western Kenya setting. This pilot study used quality improvement tools in both preparation and implementation phases, which elicited a marked increase in the number of highquality accessible trauma files and, therefore, a much clearer picture of the trauma burden presented by the potential hundreds of uncaptured trauma patients at this site.

Delta shock index in traumatic brain injury as a predictor of mortality. Zachary Warriner ${ }^{1}$, Morgan Schellenberg ${ }^{1}$, Kenji Inaba ${ }^{1}$, Marc D. Trust ${ }^{1}$, Bryan Love ${ }^{1}$, Vincent Cheng ${ }^{1}$, Aaron Strumwasser ${ }^{2}$, Demetrios Demetriades ${ }^{1}$. From the ${ }^{1}$ LAC+USC Medical Center, Los Angeles, CA; and ${ }^{2}$ University of Southern CA, Los Angeles, CA.

Background: Elevated delta shock index (DSI), defined as the change in shock index (heart rate/systolic blood pressure) over time, independently predicts mortality after trauma. However, preliminary studies suggest that the relationship between DSI and mortality is bimodal among the subset of trauma patients with traumatic brain injury (TBI). Because this relationship is incompletely defined by the current literature, the purpose of 
this study was to analyze the relationship of DSI to mortality among TBI patients. Methods: All patients presenting to our level I trauma centre (2008-2018) as trauma team activations were included. Patients with severe extracranial injuries (Abbreviated Injury Scael [AIS] score $\geq 3$ ) or missing mortality data were excluded. DSI was defined as the change in SI between prehospital and initial emergency department measurements. Mortality was compared across DSI quartiles based on pre-existing studies using $\chi^{2} /$ Fisher exact tests. Subgroup analysis was performed based on severity of head AIS and mechanism of injury. Results: Over the study period, 2770 TBI patients were identified. Median age was 41 (interquartile range [IQR] 25-58) years, and $75 \%$ of patients were male $(n=2080)$. Median Injury Severity Score on admission was 12 (IQR 5-20), with median head AIS of 3 (IQR 2-3). The majority of patients suffered a blunt mechanism of injury, constituting $93 \%$ of the study population $(n=2590)$. Overall mortality was $9 \%(n=267)$. DSI quartiles $(<-0.5,-0.5$ to $<0,0$ to $0.5,>0.5)$ showed a bimodal distribution of increased mortality with higher indices $(18 \%, 7 \%, 11 \%$, and $37 \% ; p<0.00001)$. When comparing TBI severity, this effect was not seen with mild to moderate TBI patients, who showed an overall mortality of $1 \%(n=14 ; 0 \%, 1 \%, 2 \%$, and $0 \% ; p=0.036)$. However, in the most severely injured patients, this effect was preserved $(28 \%, 13 \%, 18 \%$, and $54 \% ; p=$ $0.00018)$, with an increased overall mortality of $17 \%(n=252)$. Regarding mechanism of injury, this effect was not seen in penetrating TBI $(45 \%, 13 \%, 18 \%$, and $33 \% ; p=0.20)$, but was preserved in a blunt mechanism of injury $(10 \%, 7 \%, 11 \%$, and $36 \% ; p<0.00001)$. Conclusion: Although prior studies suggest a bimodal association between the DSI and mortality in TBI, the current study shows that this distribution is seen only in the most severely injured patients, and those who suffer a blunt mechanism of injury. Caution must be employed when using the DSI in patients with TBI because of this bimodal association with mortality.

Perioperative VTE prophylaxis in orthopedic trauma patients at Vancouver General Hospital - Is there room for improvement? Annie Lalande', Emilie Foos ${ }^{2}$, Philip Dawe ${ }^{3}$, Morad Hameed $^{3}$, David Evans ${ }^{3}$, Naisan Garraway ${ }^{3}$. From the ${ }^{1}$ University of British Columbia, Vancouver, BC; the ${ }^{2}$ Vancouver General Hospital, Vancouver, BC; ${ }^{3}$ Trauma Services - Vancouver General Hospital, Vancouver, BC.

Background: Venous thromboembolic events (VTE) are a significant cause of morbidity and mortality in trauma patients, who have an inherently higher VTE risk than the general hospitalized patient population. The VTE rate was anecdotally found to be higher than expected in patients with orthopedic trauma at Vancouver General Hospital (VGH). It was hypothesized to be in part due to variability in local practices of holding VTE prophylaxis preoperatively, and the safety of perioperative prophylaxis administration was questioned. Methods: A literature search was conducted through the PubMed and Cochrane databases. MeSH terms used were "VTE prophylaxis," "heparin," "peri-operative," "pre-operative" and "orthopedic trauma." The current practice guidelines from multiple societies, including the American Association of Orthopedic Surgeons and CHEST, were reviewed. The provincial trauma database at VGH was used to establish the VTE rate in orthopedic trauma, and current VTE prophylaxis practices among the trauma surgery staff were delineated. Results: VTE rates have been increasing yearly in trauma patients with orthopedic injuries at VGH from 2012 to 2015, to a maximum of $4.5 \%$, which is higher than the rate of our general trauma population of $1.3 \%$ (deep vein thrombosis) and $1.8 \%$ (pulmonary embolism). The current practice at VGH involves a twice daily dose of enoxaparin for patients with long bone and pelvis fractures as well as spinal cord injuries, a daily dose of dalteparin for most other trauma patients with the exception of renally impaired patients, who receive heparin. Orthopedic trauma patients with multiple other injuries are risk-stratified as being at high risk for VTE. Disruption in vascular endothelium, tourniquet application, limb immobilization, manipulation and cement application are factors specific to orthopedic surgery that contribute an additional perioperative VTE risk. Variability in guidelines exist as to optimal timing for initiation of prophylaxis, with recommendations varying from more than 12 hours up to 2 hours preoperatively, or 4-6 hours postoperatively. Through the literature review, 6 studies evaluating the efficacy and safety of intraoperative heparin administered after neuraxial analgesia were identified. The doses administered were lower than standard VTE prophylaxis, but no significant increase in major bleeding was observed. Decrease in VTE rates were not observed in all studies, but the intervention was considered to be safe. Conclusion: VTE prophylaxis administration requires evaluation of bleeding risk, but is generally safe, even in the intraoperative setting. The practice of holding the preoperative dose for operations at risk of being delayed on the emergency slate is therefore questioned. The next steps include surveying the orthopedic surgeons at VGH and external sites to review local practices and rationales, as well as openness to a change in practice for standard VTE prophylaxis to a daily dalteparin.

Mental health outcomes in children with pediatric traumatic injury compared with matched controls: a population-based, longitudinal study. Dominic Chung, Fustin Gawaziuk, Nora Cristall, Sarvesh Logsetty. From the University of Manitoba, Winnipeg, MB.

Background: Pediatric traumatic injury (PTI) is a leading cause of hospitalization among children. Little is known about subsequent mental health outcomes (MHOs). This longitudinal cohort study compared the MHOs in survivors of PTI with matched controls from the general population. This study hypothesized that survivors of PTIs will have increased rates of $\mathrm{MHO}$ relative to matched controls. Methods: Children younger than 18 years who survived an injury that required hospitalization between Jan. 1, 2004, and Dec. 31, 2016, $(n=9551)$ were matched to 5 controls from the general population $(n=47755)$ on age, sex and geographic region. Pre- and postinjury adjusted relative rates (ARRs) of anxiety, depression and substance use disorders were determined with generalized estimating equations, adjusting for sex, income group, location and age. Results: Survivors of PTIs had increased ARR of anxiety (1.66, 95\% confidence interval [CI] 1.51-1.82), depression (2.87, 95\% CI 2.57-3.21), substance use (RR 3.23, 95\% CI 2.64-3.99) and any MHO (1.90, 95\% CI 1.762.04). They also had high preinjury rates of anxiety $(1.30,95 \%$ CI 1.16-1.47), depression (2.00, 95\% CI 1.73-2.32), substance use (4.99, 95\% CI 3.08-5.20) and any MHO (1.50, 95\% CI $1.37-$ 1.66). Comparing the pre- and postinjury ARRs showed a 
significant difference over time $(p<0.001)$ for anxiety, depression and any MHO. There was no significant difference in substance use over time. Conclusion: Child survivors of traumatic injury had increased ARR of anxiety, depression and any MHO compared with matched control after adjustments for sex, income and age group and geographic location. These findings support targeted intervention strategies for this population.

What is the role of sleep hygiene injury prevention? Understanding the sleep habit of teenagers attending the P.A.R.T.Y. Program at Sunnybrook Health Sciences Centre using a quality improvement approach. Brandy Tanenbaun, Sharon Ramagnano. From the Sunnybrook Health Sciences Centre, Toronto, ON.

Background: Despite efforts to reduce preventable injury and related deaths in Canada, adolescent injury remains a social and economic concern. The effects of insufficient sleep for healthy adolescents are well established and include daytime tiredness, depression, diminished decision-making and judgment leading to injuries, and poor school and athletic performance. The purpose of this quality-improvement study is to understand the sleep hygiene behaviours of adolescent participants of the Toronto P.A.R.T.Y. Program and inform curriculum development. Methods: Inclusion criteria included high school students, aged 16 years or older, who attended the Toronto P.A.R.T.Y. Program between May 1 and May 18, 2018. The Adolescent Sleep Hygiene Scale (ASHS) was used to assess sleep practices theoretically important for optimal sleep. Participants completed the ASHS during the P.A.R.T.Y. Program online or using a paper version. Microsoft Excel was used for coding, and descriptive analysis was performed using SPSS version 21. Results: The sample included 136 consenting participants. The final sample included 111 adolescents (mean age $16.7 \pm 0.73$ (range 16-18) yr; males and females, $80 \%$ public schools in the greater Toronto area). A 6-point ordinal rating scale ranging from 1 (never) to 6 (always) was used. Subscale score was calculated taking the average of the items comprising that subscale, and the mean of the subscale scores is the total sleep hygiene score. The mean score for sleep hygiene was $3.66 \pm 0.58$. Behavioural factor $(2.57 \pm$ $1.01)$, sleep stability factor $(2.63 \pm 1.18)$ and daytime sleep factor $(2.93 \pm 0.87)$ scored the lowest, while substance factor $(5.26 \pm$ $0.94)$ and sleep environment factor $(4.77 \pm 0.79)$ scored the highest. Adolescents scored the lowest in these key areas: "I use my bed for things other than sleep," "I go to bed and think about things I need to do," and "On weekends, I stay up more than 1 hour past my usual bedtime." There is no published data interpretation for what should be considered as optimal or suboptimal sleep hygiene. Therefore, we used quintiles of the sample distribution of the total ASHS score in an extreme-groups analysis to compare adolescents with suboptimal sleep hygiene (ASHS score $\leq 3.25$ ) to optimal sleep hygiene (ASHS score $\geq 4.12$ ). While the mean score suggests the majority have some healthy sleep hygiene behaviours, $75 \%$ of the sample scored below optimal, suggesting an opportunity for future P.A.R.T.Y. Program intervention. Conclusion: Sleep hygiene is a modifiable risk factor for injury. P.A.R.T.Y. Program participants report practising some healthy sleep behaviours, but not sufficiently to modify injury risk. This study suggests there is a need to include sleep hygiene content in future P.A.R.T.Y. Program curriculum. In particular, there should be emphasis on behavioural arousal factor, sleep stability factor and daytime sleep factor.

Readiness for chest tube insertion among surgical and nonsurgical trainees. Tanya Kuper ${ }^{1}$, Nick Federman ${ }^{2}$, Patrick Murphy $^{1}$, Neil Parry ${ }^{2}$, Rob Leeper ${ }^{1,2}$. From ${ }^{1}$ Western University, London, $\mathrm{ON}$; and the ${ }^{2}$ London Health Sciences Centre, London, $\mathrm{ON}$.

Background: There has been a recent shift in medical education toward competency-based training. Central to this design are entrustable professional activities (EPAs), such as the insertion of a surgical chest tube during an intensive care unit (ICU) rotation. We evaluated resident readiness for chest tube insertion using a high-fidelity simulator and validated assessment tool. We aimed to identify areas of weakness to inform curriculum development and identify residents at increased need for simulation-based training and supervision. Methods: Residents of all training levels before their ICU rotation or currently rotating through the ICU were invited to participate in the study. Participants were shown a standardized instructional video created by the research team before completing a preprocedural checklist and inserting a thoracostomy tube on a Trauma Man simulator. Performances were recorded and scored by a blinded, naive rater using the validated TUBE-iCOMPT rubric. Placement within the pleural cavity was confirmed. Results: Forty-nine residents participated in the study; 30 were from nonsurgical and 19 from surgical training programs. All surgical trainees were Advanced Trauma Life Support (ATLS)-certified, while only $50.0 \%$ of nonsurgical trainees had received their ATLS certification. In general, surgical trainees were more junior than nonsurgical trainees $(21.1 \%$ v. $36.7 \%$ were senior residents, respectively) and had placed more chest tubes before participation in the study (median chest tube placement 2 [interquartile range (IQR) 1-15] v. 1 [IQR 0-3], respectively). Surgical trainees demonstrated higher procedural skill at chest tube insertion than their nonsurgical counterparts (median total TUBE-iCOMPT score among surgical trainees 88/100 [IQR 74-90] v. 75/100 [IQR 66-85] among nonsurgical trainees, $p=$ $0.01)$. Nonsurgical trainees in particular were rated more poorly in the patient positioning and blunt dissection subdomains of the TUBE-iCOMPT rubric ( $p=0.01$ and $p=0.03$, respectively). Additionally, surgical trainees were more likely to place the chest tube correctly in the pleural cavity than nonsurgical trainees (84.2\% v. 63.3\%, respectively); however, this did not reach statistical significance $(p=0.11)$. Finally, there was no significant difference in procedural duration among surgical than nonsurgical trainees (243 s v. 233 s, $p=0.49$ ). Conclusion: Surgical trainees demonstrated higher chest tube insertion skills than nonsurgical trainees despite being more junior. This likely is related to ATLS training and familiarity with chest tube simulators, rather than marginally increased chest tube insertion experience. Furthermore, time was a poor measure of competence in our study. Formal, simulation-based training for chest tube insertion and increased supervision of residents without previous ATLS training likely is of benefit before and during ICU rotations.

Creating a physical telementored ultrasound supported medical interventions (TMUSMI) "box." Fessica Mckee", Paul McBeth $^{1}$, Juan Wachs ${ }^{2}$, Douglas Hamilton ${ }^{3}$, Chad Ball ${ }^{1,4}$, Lawrence Gillman ${ }^{3}$, Andrew Kirkpatrick ${ }^{1,4}$. From the 
${ }^{1}$ University of Calgary, Calgary, AB; ${ }^{2}$ Purdue University, West Lafayette, IN; the ${ }^{3}$ University of Manitoba, Winnipeg, $\mathrm{MB}$; and the ${ }^{4}$ Foothills Medical Centre, Calgary, AB.

Background: Exsanguination typically occurs in prehospital settings, most often from noncompressible torso hemorrhage. Remote telementoring mentored interventions (RTMIs) permit remote expert guidance of nonsurgeons to diagnose and intervene in catastrophic situations. Our group recently broadened our scope beyond telementored ultrasound diagnoses in an attempt to support all phases of a remote trauma resuscitation with life-saving interventions. As no commercial off-the-shelf (COTS) telemedical instrumentation package appeared ideal to support such an endeavour, we created a prototypical instrumentation-box to support RTMIs. Methods: The prototypical box consisted of a tablet (Samsung Galaxy Tab E 8.0", LTE, Samsung Electronics) with redundant (cellular and wifi) connectivity providing communications. A head-mounted point-of-view camera and microphone provided 2-way voice and unidirectional video communication. A remote graphic user interface allowed remote mentor activation and remote control of the video laryngoscope (Rusch Airtraq, Teleflex), portable ultrasound (Lumify, Philips), and a vital signs monitor (electrocardiographic/oxygen saturation/blood pressure capabilities; Feel and Air, iHealth Laboratories Inc). Three children (ages 6, 13, and $15 \mathrm{yr}$ ) were remotely guided to conduct a simulated resuscitation by subject-matter experts. Results: Remote activation of the central control panel, vital signs monitor, and remote ultrasound knobology was demonstrated even with a mentor onboard a commercial aircraft flight. Using the box, a 6-year-old in Edmonton was mentored from Orembro, Sweden, to confirm breathing and to rule out pneumothorax by demonstrating sonographic lung sliding, power-slide sign, and sea-shore sign requiring remote activation of both colour-power Doppler and M-mode functions. Thereafter, the same child was mentored to demonstrate normal ventricular contractility and to rule out a pericardial effusion. Subsequently, 13- and 15-year-old secondary school students were successfully mentored by a remote critical care physician to intubate a laryngeal simulator (AirSim Standard, TrueCorp) with a video laryngoscope despite having no prior knowledge of airway anatomy nor exposure to the equipment. In this scenario, the remote mentor instructed the students to apply the blood pressure monitor and oxygen saturation probe and initiated the remote monitoring of these parameters. To enable remotely mentored intubation, a dual video feed involving split screens, with the upper screen displaying the students heads-up view demonstrating their hands and the simulated airway, and the lower screen demonstrating the endoscopic depiction of the airway, was found to be invaluable by the remote mentor. Thus, the RTMI box successfully allowed for definitive airway capture, ruling out of pneumothorax and confirmation of bilateral lung ventilation, and confirmed grossly normal cardiac contractility when performed by students guided by distant remote physicians. Conclusion: An RTMI telemedicine instrumentation package consisting of COTS components was able to support essential elements of a simulated resuscitation when conducted by students as young as 6 years old. Remote experts were not only able to communicate and guide from afar, including in-flight, but also to troubleshoot, activate, and adjust the knobology of the medical equipment the students used. Although further technical refinements are required to ruggedize, economize, and standardize this plat- form that should be delivered to clinicians for real-world validation and implementation.

Pedicatric trauma age cut-off: When and where is the best care? Ioana Bratu, Sukbdeep Dulai, Cathy Falconer. From the University of Alberta and Stollery Children's Hospital, Edmonton, AB.

Background: Across the country, there is no agreed age cut-off of what defines a pediatric trauma patient. This has great implications for orthopedic injuries, as the "pediatric-type" orthopedic injuries are managed differently than the more "adult-like" orthopedic injuries. The adult-like orthopedic injuries require a different set of surgeon skills and resources that are provided outside the pediatric operating room (OR) in the adult OR. Methods: Recent pediatric traumas requiring orthopedic intervention were reviewed at our institution. Particular attention was made to the different resource needs of patients with pediatrictype fracture versus the teenager with adult-like fracture. We focused on patients aged 14-18 years going forward since January 2018, when our institution changed its admitting policy to 18 years of age. Results: During past year, there were 13510 pediatric trauma patients assessed in our institution's emergency department, of which 975 were admitted. Of those admitted, 104 had an Injury Severity Score $>12$, of which half are in the teenage group (14-18 yr). After inception of age cut-off at 18 years for our institution, from January to May 2018, there were 240 pediatric trauma cases of all ages requiring access to the OR. Of those, $90 \%(214 / 240)$ where in the teenage group. Fortythree percent (93 of 214) of the teens who needed access to the OR required an orthopedic procedure. Around $6.5 \%$ of those teens requiring an orthopedic intervention required access to the adult-like orthopedic OR. Conclusion: Teens required most access to pediatric OR resources, many of which were orthopedic operations, with a small but important group requiring access to the adult OR for adult-like orthopedic injuries. Planning for appropriate age-related inpatient and OR resources is paramount, being mindful of patient and family support. Standardization of Canadian age cut-off for pediatric trauma patients should be sought so that the best care can be provided to the right patient at the right place.

Trauma survivor night: resilience, recovery, recognition. Amy Makish, Meaghan McLachlin, Alison Armstrong, Neil Parry, Kelly Vogt. From the London Health Sciences Centre, London, ON.

Background: The trauma experience does not end once a trauma survivor is discharged from the acute care facility. We hypothesized that there is value in continuing the relationship between patients, family and hospital providers to celebrate resiliency, share the narrative of recovery and to recognize the survivor, family and health care provider's role in trauma. A trauma survivor night was conducted to reunite patients, family and providers to celebrate survival and share stories of recovery. Methods: A trauma survivor night was held, inviting patients, family members, and health care providers from the lead trauma hospital. The evening included guest speakers discussing caregiver fatigue and warnings signs of posttraumatic stress disorder; time for trauma survivors to share their story; and an opportunity for 
survivors, family and providers to reconnect, celebrate recovery, and express appreciation. The event was evaluated using surveys provided to survivors, family, and health care providers. Results: Trauma patients $(n=71)$ admitted within the previous year were invited along with their families. In attendance were $23 / 71$ (32.4\%) patients and 41 family members, with equal representation from the local city and surrounding area. Survey response rate was $83 \%$ for patients $(n=19)$ and $54 \%$ for family $(n=22)$. Patients and family members reported that the event provided them with answers to gaps in their story, realization that they were not alone in their survival, an opportunity to celebrate their journey, and closure to their trauma. Hospital staff from nursing, physician and allied health providers were invited from the emergency department, critical care and trauma ward, with 50 staff attending and a survey response rate of $54 \%(n=27)$. The staff indicated they were hesitant to attend the inaugural event, as they were not certain of the benefit, but found the event provided them with the chance to reconnect with patients and see the patients' progress. Visualizing how well their patients were recovering reminded providers of the importance of their work. Most staff $(70 \%)$ rated their joyfulness in the work place as moderate, but the majority (89\%) found that this type of event would improve their joyfulness in the workplace. Patients, family and providers were unanimous $(100 \%)$ that this was a successful event, would attend the event again, and would encourage others to attend in the future. Conclusion: Our trauma program successfully implemented a trauma survivor night that patients, family members, and health care providers felt was a valuable experience. This night celebrated survival and resiliency, promoted ongoing healing for the patients, honoured the role of family as caregiver, and improved joyfulness in the workplace for health care providers. Trauma survivor night will now be an annual event at our institution.

Trauma surgery exposure among general surgery residents at McMaster University: a retrospective case log analysis. Andrew Versolatto ${ }^{1}$, Qian Shi ${ }^{1}$, Angela Coates ${ }^{2}$, Paul Engels ${ }^{1}$, Timothy Rice ${ }^{1}$. From ${ }^{1}$ McMaster University, Hamilton, ON; and the ${ }^{2}$ Hamilton Health Sciences Trauma Program, Hamilton, ON.

Background: Being competent to provide operative trauma care is a mandatory objective of Canadian general surgery resident training. The Royal College of Physicians and Surgeons of Canada (RCPSC) does not specify a minimum number of trauma cases during training; the Accreditation Council for Graduate Medical Education (ACGME) in the United States requires a minimum of 10 . To better assess the adequacy of operative trauma exposure during residency, we constructed a retrospective trauma operative case log for McMaster University residents over the last decade. Methods: The Hamilton General Hospital (HGH) Trauma Registry was used to identify all patients from July 2008 to June 2018 who underwent a nonorthopedic/nonneurosurgical trauma procedure. Hospital records were reviewed to determine the type of procedure and which residents and staff were present at each operation. A list of residents was obtained from the McMaster general surgery residency program and was cross-referenced with those trainees present at the operation. This project received research ethics board approval. Results: During the 10-year study period 417 patients underwent a total of 561 surgical procedures (including 306 index laparotomies, 87 second-look laparotomies, and 88 thoracotomies). The mean number of cases per annum at $\mathrm{HGH}$ for specific surgical procedures was splenectomy 7.2, liver repair/resection 3.1, bowel resection 8.9 , diaphragm repair 5.4 , retroperitoneal exploration 2.3 , pancreatic resection 0.7 , duodenal repair 0.6 , cardiac repair 1.1 , renal repair/resection 0.4 , lung resection 0.8 , major vascular repair 1.9 , and neck exploration 1.9. The start times for trauma operations were distributed as follows: $25 \%$ weekday daytime (8 am to $5 \mathrm{pm}$ Monday to Friday); $31 \%$ weekday overnight (5 pm to 8 am Monday to Thursday), and $43 \%$ weekends ( 5 pm Friday to 8 am Monday). There were 123 residents enrolled during the study period, with a mean of 8 per postgraduate year (PGY). A resident was present at each procedure $81 \%$ of the time. The mean cumulative number of trauma procedures exposed to according to PGY level was PGY1 3.65, PGY2 4.17, PGY3 7.10, PGY4 6.83, and PGY5 8.74. For residents who completed their entire general surgery residency training during this study period $(n=35)$, the median cumulative number of index trauma laparotomies attended was 5 . The distribution of these index trauma laparotomies was as follows: 5 residents present for $\geq 10$ cases, 20 residents present for 5-9 cases, 13 residents present for $<5$ cases (2 residents at only 1 case each). Conclusion: Opportunities for operative trauma exposure among general surgery residents at McMaster University predominantly occur after hours, are limited in number, and are not fully attended. The cumulative operative procedure exposure among graduating residents is inadequate by ACGME standards and raises questions about competency in the Canadian context. Further research on trauma education during residency, including other RCPSC training sites, is recommended.

Stop the bleed: a Canadian response to improve public knowledge and confidence with uncontrolled bleeding recognition and intervention. Sharon Ramagnano, Avery Nathens, Dee Naidu. From the Sunnybrook Health Sciences Centre, Toronto, ON.

Background: In response to events in the United States, the American College of Surgeons launched Stop the Bleed to provide civilian bystanders the skills and tools to identify and stop uncontrolled bleeding and save lives. In May 2017 an interprofessional team from Sunnybrook Health Sciences Centre in Toronto set out to bring this program to Canada by setting up public courses as well as high school programs to improve public knowledge and confidence with uncontrolled bleeding. Methods: The program was delivered by 2 or more health care professionals in settings including 8-160 participants. Training was provided to the public in high-risk locations, such as sports arenas and public transit, as well as the general public and high school students interested in a career in health sciences. Approximately 700 public members were evaluated with a pre/ post survey addressing confidence level with bleeding and willingness to help a stranger. Results: Pre and post surveys were developed by the interprofessional core team, which included content experts such as trauma surgeons, intensivists, trauma team leaders, physician assistants and nurses. Only participants who completed both the pre and post surveys were included in the study. The results showed the confidence level increased from $32 \%$ to $99 \%$ in the public group and increased from $43 \%$ 
to $100 \%$ in the high school group. Willingness to help a stranger improved from $81.5 \%$ to $98 \%$ in the public group and from $68 \%$ to $98 \%$ in the high school group. Conclusion: The results showed a significant change, in that the course successfully improved public knowledge and confidence with uncontrolled bleeding recognition and intervention. The program should be part of trauma program outreach. Sunnybrook has been able to bring this program to other organizations across the country by training other instructors, resulting in national dissemination where courses are now up and running in Ottawa, Kingston, Newfoundland, Alberta, New Brunswick and British Columbia, and Quebec will be soon.

Association between leisure activity and risky driving behaviour in young Canadians (16-24 years of age). Vabid Mebrnoush $^{1}$, Jeff Brubacher ${ }^{2}$, Herbert Chan ${ }^{2}$, Shannon Erdelyi ${ }^{2}$. From the ${ }^{1}$ University of British Columbia, Vancouver, BC; and the ${ }^{2}$ Vancouver General Hospital, Vancouver, BC.

Background: Motor vehicle crashes (MVCs) are the leading cause of injury-related death in youth and impose a huge burden on the health care system and community. Risky driving behaviour (RDB) is a major risk factor for MVCs and may be impacted by leisure activity. We investigated the relationship between leisure activities and RDB in young Canadians while controlling for personality traits, socioeconomic status, demographic characteristics, and general driving information. Methods: We engaged a 20-member youth advisory committee (YAC) to develop a cross-sectional online survey-based study. The YAC provided their opinion on all steps of survey development and content (e.g., list of leisure activities, rewording of materials for a youth audience, and best platform to approach young Canadians). The survey included questions on demographics, leisure activity, driving behaviour, and personality traits. It was available to Canadians aged 16-24 years through Facebook for a 3-month period. Results: A total of 1068 young adults (mean age $18.34 \pm 2.31 \mathrm{yr}, 63.5 \%$ male) responded. Of these young drivers, $47 \%$ were high-risk, $32 \%$ moderate-risk and $21 \%$ were low-risk. The high-risk drivers were aged $18.75 \pm 2.23$ years, primarily male $(73.7 \%)$, with postsecondary education $(39.5 \%)$, and they had their own cars (76.7\%). Lowrisk drivers were aged $17.38 \pm 2.11$ years, primarily female $(57.3 \%)$, had not completed high school (74.2\%), and did not have their own cars $(80.9 \%)$. Most high-risk drivers came from families that owned $\geq 2$ cars $(54.6 \%)$ compared with only $27.6 \%$ of families of low-risk drivers. Generally, study participants engaged in video games, sports, watching movies, family/friend-involved activities, drug/alcohol-involved activities, art, writing/reading-involved activities, volunteering, listening to music, and watching TV at low levels, except for social media at high level. High-risk drivers seem to engage more than low-risk drivers in collision sports such as hockey (34.5\% v. 12.9\%), social events with friends $(22.5 \% \mathrm{v}$. $12.2 \%)$, sport/recreation volunteering (24.8\% v. $10.1 \%)$, listening to rap/electronic music ( $40.6 \%$ v. $26.7 \%)$, psychedelics $(1.9 \%$ v. $0.0 \%)$, prescription drugs (2.9\% v. $0.0 \%)$, and drinking hard liquor (34.8\% v. 23.8\%). Moreover, there were more high-risk than lowrisk drivers in the group with high engagement in alcohol-involved activities $(8.8 \%$ v. $1.3 \%)$ and in drug-involved activities $(15.7 \%$ v. $3.1 \%)$. Conclusion: This study casts light on the RDB of young Canadians. It extends the knowledge about RDB, leisure activities and their possible associations considering sociodemographic and personality traits. Our findings may inform the development of awareness campaigns and effective prevention strategies.

Hemorrhage from pelvic fracture: Can Trendelenburg positioning help? Danby Kang ${ }^{1}$, fobn Kubasiak ${ }^{2}$, Faran Bokbari $^{2}$, Matt Kaminsky ${ }^{3}$. From the ${ }^{1}$ Rush University Medical Center, Chicago, IL; the ${ }^{2}$ Cook County Hospital, Chicago, IL; and the ${ }^{3}$ Cook County Trauma Unit, Chicago, IL.

Background: Pelvic traumas maintain a mortality of $8 \%-10 \%$, $90 \%$ of which is due to venous hemorrhage. Physiologically, bleeding stops when venous and intrapelvic pressure equalize. Open pelvic fractures create more space that decreases intrapelvic pressure, contributing to pressure imbalance and therefore venous blood loss. The head-down position may have a role in pelvic trauma as a method of local hemorrhage control in patients with pelvic fracture by lowering the pelvic venous pressure (PVP). Methods: We prospectively enrolled 15 patients who have sustained 1 or more traumatic injuries and required central venous access via iliofemoral vein. Patients were placed in a semirecumbent position at $30^{\circ}$, supine position, and $-5^{\circ}$ and $-10^{\circ}$ of Trendelenburg position. At each angle, the transducer was placed at the level of anterior superior iliac spine and PVP was measured. The data were analyzed using a paired $t$ test. Results: The mean PVP at $30^{\circ}$ semirecumbent position was $18.5 \pm 4.5 \mathrm{~mm} \mathrm{Hg}$, at supine position was $14.9 \pm 4.3 \mathrm{~mm} \mathrm{Hg}$, at $-5^{\circ}$ was $12.6 \pm$ $4.4 \mathrm{~mm} \mathrm{Hg}$, and at $-10^{\circ}$ was $11.5 \pm 4.5 \mathrm{~mm} \mathrm{Hg}$. The mean PVP had decreased significantly from semirecumbent to supine position $(p<0.001)$ as well as from supine position to $-5^{\circ}$ Trendelenburg $(p<0.001)$, and from $-5^{\circ}$ to $-10^{\circ}$ Trendelenburg $(p<0.001)$. Conclusion: There was a significant PVP reduction with each change in patient tilt in the head-down position. Trendelenburg position is a fast and simple manoeuvre that effectively lowers PVP. It does not require additional training and is easy to monitor. When used in the initial resuscitative period, Trendelenburg positioning can help reach the intrapelvic and venous pressure equilibrium quickly. Prompt institution of this manoeuvre will facilitate hemorrhage control in patients with pelvic fracture.

Low-value clinical practices in the trauma intensive care unit: a scoping review and expert consultation study. Lynne Moore $^{1}$, François Lauzier ${ }^{2}$, Pier-Alexandre Tardif ${ }^{1}$, François Lamontagne ${ }^{2}$, Michael Chassé $e^{3}$, Henry Thomas Stelfox ${ }^{4}, \mathcal{F o h n}$ Kortbeek $^{4}$, Paule Lessard-Bonaventure ${ }^{1}$, Catherine Truchon ${ }^{5}$, Alexis Turgeon'. From the ${ }^{1}$ Université Laval, Québec, QC; the ${ }^{2}$ Axe Santé des Populations - Pratiques Optimales en Santé, Traumatologie - Urgence - Soins intensifs, Québec, QC; the ${ }^{3}$ Université de Montréal, Montreal, QC; the ${ }^{4}$ University of Calgary, Calgary, AB; the ${ }^{5}$ Institut national d'excellence en santé et services sociaux, Québec, QC; and the ${ }^{6} \mathrm{CHU}$ de Québec Research Center, Université Laval, Québec, QC.

Background: Injuries lead to 200000 hospital stays, 60000 disabilities, and 13000 deaths per year in Canada, with direct costs of $\$ 16$ billion. Low-value medical interventions are estimated to consume up to $30 \%$ of health care resources and may expose patients to avoidable harm. However, little is known about health care overuse in trauma populations. We aimed to identify lowvalue clinical practices in the trauma intensive care unit (ICU). 
Methods: We conducted a scoping review targeting research articles, reviews, recommendations and guidelines that identified at least 1 low-value clinical practice specific to injury care. Pairs of reviewers evaluated eligibility and extracted data using a prepiloted form. We then asked critical care physicians to rate practices on a 5-point Likert scale from clearly low-value to clearly beneficial. These categories mirror the "clearly ineffective, grey zone, and clearly effective" classifications described in the Lancet Right Care series. Results: Of 72226 citations identified in the scoping review, 815 met our inclusion criteria and led to the identification of $41 \mathrm{ICU}$ practices. Of 8 critical care physicians we approached, all completed the consultation survey. We identified 15 clinical practices that met study criteria for low-value care; that is, they were identified in a study providing level I, II, or III evidence and at least $75 \%$ of experts considered them clearly or potentially low-value. These included 4 related to medications (corticosteroids, antibiotics prophylaxis and antiseizure prophylaxis) and 4 related to fluids and blood products (albumin, colloids, platelet and red blood cell transfusion). Twenty-six practices were in the "grey zone," including decompressive craniotomy and hourly neurologic assessments $>24$ hours for stable traumatic brain injury. Conclusion: We identified 15 lowvalue clinical practices in the trauma ICU that are supported by empirical evidence and expert opinion. Conditional on the results of future research, they represent potential targets for guidelines, overuse metrics and de-implementation interventions. We also identified 26 "grey zone" practices, which may be interesting targets for value-based decision-making. Our study represents a crucial step toward the de-implementation of low-value clinical practices in injury care.

Frostbite injuries: independent predictors of outcomes. Morgan Schellenberg ${ }^{1}$, Vincent Cheng ${ }^{1}$, Kenji Inaba ${ }^{2}$, Christopher Foran ${ }^{1}$, Zachary Warriner ${ }^{1}$, Marc D. Trust ${ }^{1}$, Damon Clark ${ }^{1}$, Demetrios Demetriades ${ }^{1}$. From the ${ }^{1}$ LAC+USC Medical Center, Los Angeles, CA; and the ${ }^{2}$ Division of Trauma and Surgical Critical Care, Los Angeles, CA.

Background: Frostbite injuries are infrequent but important causes of morbidity and mortality after trauma. In part because of their rarity, frostbite injuries are incompletely defined by the current literature in terms of patient characteristics, injury patterns, and outcomes. This study defined the epidemiology, injuries, and independent predictors of outcomes after frostbite trauma. Methods: All patients presenting to hospital after frostbite injury based on Abbreviated Injury Scale (AIS) codes were identified from the National Trauma Data Bank (NTDB; 2007-2014). Demographics, injury data, interventions, and outcomes were collected. Patients were dichotomized into study groups based on intensive care unit (ICU) admission. Variables were compared using the Mann-Whitney $U$, Fisher exact, or $\chi^{2}$ tests as appropriate. Multivariable analysis using logistic regression was used to determine independent predictors of outcomes (mortality, need for amputation, and hospital length of stay [LOS]). Results: Over the study period, 241 patients $(<0.01 \%$ of the NTDB) were identified. Median age was 44 (interquartile range [IQR] 29-58) years, and $76 \%$ of patients were male $(n=184)$. Median body temperature on admission was $36.3^{\circ} \mathrm{C}$. Mortality was $3 \%(n=7)$, with median hospital LOS of 5 (IQR 2-10) days and median ICU LOS of 0 (IQR 0-0) days. Amputation was required in $5 \%$ of patients $(n=13)$. On univariate analysis, ICU admission $(n=101,42 \%)$ was associated with lower body temperature $\left(34.1^{\circ} \mathrm{C}\right.$ v. $36.6^{\circ} \mathrm{C}, p<$ 0.001 ), lower Glasgow Coma Scale (GCS) score (13 v. 15, $p<$ 0.001 ), and higher Injury Severity Score (ISS; 14 v. $5, p<0.001$ ). On multivariate analyses, mortality was independently predicted by lower GCS on admission (odds ratio [OR] 0.776, 95\% confidence interval [CI] 0.620-0.971, $p=0.027$ ). The need for amputation was independently predicted by higher heart rate (OR 1.040, $95 \%$ CI $1.008-1.073, p=0.013)$. Finally, higher heart rate $(\mathrm{OR}$ $0.011,95 \%$ CI $0.005-0.017, p<0.001$ ) and ISS (OR 0.050, 95\% CI $0.035-0.065, p<0.001$ ) were independent predictors of hospital LOS. Conclusion: Frostbite injuries are uncommon but can necessitate amputation and cause mortality. Poorer outcomes (mortality, amputation, need for ICU admission, and longer hospital LOS) are predicted by lower body temperature and GCS as well as higher ISS and heart rate on admission. These findings can be used to triage patients appropriately upon presentation to hospital and to better inform prognosis after frostbite injuries.

Evaluating the impact and content of standardized feedback from a lead trauma hospital to referring hospitals. Matt LeBreton $^{1}$, Kiera Levesque ${ }^{2}$, Facinthe Lampron ${ }^{1}$. From the ${ }^{1}$ The Ottawa Hospital, Ottawa, ON; and the ${ }^{2}$ University of Ottawa, Ottawa, ON.

Background: The American College of Surgeons tasked level I trauma centres to provide feedback to referring hospitals (RH). In March 2017, our trauma program established a standardized written feedback (SWF) process after each $\mathrm{RH}$ transfer in the format of a written letter. We are not aware if guidelines exist for content or delivery of feedback. The objectives are to understand how this feedback is used and evaluate which data points are most useful to RH. Methods: For each trauma referral, a letter is sent to emergency department (ED) heads at the $\mathrm{RH}$, the treating physician and the Regional Trauma Network (RTN) representative. Content of the SWF was developed by trauma program staff. To evaluate these efforts, an electronic survey was developed, piloted and distributed in October 2018 to all physicians in the RTN. A reminder was sent after a 1-week period. Questions related to the letter's use and content. Results: Between April 2017 and April 2018, 333 SWF letters were sent via Canada Post based on 256 referred trauma patients from $13 \mathrm{RH}$. Out of 220 physicians, $36(16.4 \%)$ responded to the survey. Of those, 8 were ED heads, 25 were staff physicians and 3 were locum physicians. Most (96\%) responded that the SWF was a valuable trauma qualityimprovement strategy. More than half $(56 \%, n=20)$ of respondents received 1 or more feedback letters. More than half of the physicians $(56 \%, n=20)$ went and reviewed the chart and reflected on the care provided after having received the feedback. A peer-to peer discussion took place $29 \%$ of the time. The SWF triggered grand rounds or mortality \& morbidity review on 2 occasions. When asked which data point would be most useful, list of injuries (44\%) and disposition of the patient (33\%) were chosen as the 2 most useful. As for performance improvement (PI) metric, $63 \%$ identified deviations of Advanced Trauma Life Support protocol as the most useful PI metric, followed by transfer times for major trauma (35\%). Barriers to feedback consistency are related to documentation not being forwarded with the patient when transferred and legibility of $\mathrm{RH}$ documentation where 
electronic documentation was not present. Conclusion: An SWF process is a pertinent strategy that promotes conversation and quality improvement in trauma care. Although significant interest for feedback on trauma patient outcomes and process exists, tailoring data provided to referring centres could improve satisfaction and pertinence of the feedback. An automated system would benefit a trauma program to provide a broader cohort of referrals. Barriers to assuring consistent feedback is multifactorial.

Team performance in the trauma bay: identifying the need for standardization of duration, structure, and information content in clinical handovers between emergency medical services and the trauma team. Arshia P. Favidan ${ }^{1}$, Avery Nathens ${ }^{1}$, Homer Tien', Luis Teodoro Da Luz'1. From ${ }^{1}$ Sunnybrook Health Sciences Centre, Toronto, ON; and ${ }^{2}$ Ornge, Mississauga, ON.

Background: Clinical handover between emergency medical services (EMS) and the receiving hospital trauma team can be susceptible to critical errors that may negatively affect patient care. Thus far, there have been no evaluations of the quality of EMS handover to trauma teams in a Canadian setting. As such, we sought to characterize handover practices from EMS to the trauma team, identify areas for improvement, and determine if there is a need for standardization of current practices. Methods: Over a consecutive 9-week period in the trauma bay at Sunnybrook, a trained researcher collected handover data prospectively in a randomized manner. A previously validated handover framework was used to guide data collection. Categories of metrics collected included information shared, structure, and duration of the handover, as well as questions, interruptions, and perceptions of EMS, nurses, and trauma team leaders (TTLs) about the quality of the handover according to a bidirectional Likert scale. Results: Of 410 trauma team activations, 79 verbal handovers were observed. Most handovers occurred following patient transfer $(77 \%)$, and information most often communicated included any mention of identification (99\%), mechanism of injury (96\%), injuries and/or symptoms (97\%), and signs in the form of initial assessment of either airway, breathing, circulation, or disability (92\%). However, information was often missing regarding airway status (22\%), breathing status (54\%), medications (59\%), and allergies (54\%). Handover structure lacked consistency beyond the order of identification and mechanism of injury, and only $28 \%$ of handovers had a dedicated question and answer period. In terms of structure, EMS returned to categories of information unprompted in $84 \%$ of handovers. There was significant repetition of information, with $35 \%$ of all questions requesting information that had already been given. Most handovers (61\%) involved parallel conversations among the trauma team, an indication of a lack of active listening. The presence of parallel conversations was associated with a greater number of interruption questions from the trauma team $(3.15 \mathrm{v} .1 .818, p=0.001)$. There was statistically significant disparity between the perceptions of EMS regarding the self-evaluation of their handovers and the associated evaluations of nurses and TTLs in terms of the amount of information, duration, and structure of the handover. Generally, TTLs were most critical of the handovers for these 3 metrics and EMS were the least critical. Conclusion: At our trauma centre, we have identified the need for handover standardization owing to poor communication of information, a lack of structure, information repetition, a lack of active listening, and discordant expectations between EMS and nurses and TTLs. We intend to use our results to guide the development of a coconstructed framework integrating the views of all members of the trauma team and emergency medical services.

Association of geriatric trauma consultation services with acute care outcomes and resource use in traumatically brain-injured patients: a propensity score matched observational cohort study. Gwen Schwartz ${ }^{1}$, Ruiwei fing ${ }^{1}$, Amanda McFarlan ${ }^{2}$, Mingyao Liu ${ }^{1}$, Beate Sander ${ }^{3}$, Rob Fowler ${ }^{1}$, Sandro $R_{i z o l i}$. From the ${ }^{1}$ University of Toronto, Toronto, ON; ${ }^{2}$ St. Michael's Hospital, Toronto, ON; and the ${ }^{3}$ University Health Network, Toronto, ON.

Background: Traumatic brain injury (TBI) is a leading cause of hospitalization with poor prognosis among geriatric patients. Integration of geriatric trauma consultation service (GTCS) improves quality of acute care management and survivor dispositions; however, the effects of GTCS in a TBI population are poorly described. Our aim was to measure independent associations between GTCS and discharge outcomes and resource use, and to assess components of hospital length of stay (LOS) for geriatric TBI admissions. Methods: We identified geriatric (age $\geq 65$ yr.) patients admitted with TBI (Abbreviated Injury Scale [AIS]-head score $\geq 3$ ) between 2008 and 2016 at our level I trauma centre. Patients with an LOS $<2$ days were excluded to adjust for GTCS referral bias. GTCS patients were matched to those without GTCS (usual care) using propensity scores (PSM) derived from patient-level characteristic variables. Betweengroup comparisons were assessed by absolute standardized differences $(d)$. Unadjusted and PSM analyses measured associations between GTCS and outcomes. Results: Of 1152 geriatric TBI admissions, 242 were managed with GTCS. We matched $88 \%(n=213)$ GTCS patients with 213 usual care patients (PSM cohort). Lower head injury severity (maximum AIS: $4.0 \pm 0.76 \mathrm{v}$. $4.4 \pm 0.66, d=0.41)$, greater presence of concomitant extracranial injury $(36.6 \%$ v. $13.6 \%, d=0.43)$, increased trauma team activation $(72.8 \%$ v. $22.1 \%, d=0.95)$, and less neurosurgical management $(30.0 \%$ v. $73.7 \%, d=0.79)$ differentiated GTCS patients. Overall, mortality rates were $13.6 \%$ for GTCS and $14.1 \%$ for usual care patients (odds ratio [OR] 1.04, 95\% confidence interval $[\mathrm{CI}] 0.69-1.56, p=0.864)$. GTCS management was associated with increased total hospitalization days (incident rate ratio (IRR) 2.05, 95\% CI 1.77-2.37) and complication rates (37.2\% v. 20.6\%, OR 2.29, 95\% CI 1.69-3.11). Within the PSM cohort, GTCS was not significantly associated with mortality or increased in-hospital complications but was significantly associated with prolonged total and alternative level of care (ALC)LOS (IRR 1.57, 95\% CI 1.23-1.91 and IRR 1.23, 95\% CI $0.75-$ 2.02, respectively) and shorter ICU LOS (IRR 0.84, 95\% CI 0.65-1.08). Significant associations between GTCS and increased ICU LOS (OR 2.74, 95\% CI 1.82-4.13) and mechanical ventilation management (OR 1.54, 95\% CI 1.04-2.27) were evidenced, but surgical management was decreased (OR 0.60, 95\% CI 0.41-0.88). Among GTCS survivors, there was significant increased disposition to in-patient rehabilitation (OR 1.99, 95\% CI 1.29-3.08). In-hospital complications, mechanical ventilation, ICU and ALC management, injury severity, and presence of concomitant extracranial injury were the most important 
variables associated with prolonged LOS among survivors. Conclusion: GTCS management among a TBI cohort is largely partial to patients presenting with extracranial injuries in addition to TBI. Our findings suggest GTCS associations with prolonged LOS, extended stays in lower-level care (ALC), and increased disposition to inpatient rehabilitation may be influenced by increased trauma load of their managed TBI patient population. Further studies of TBI patients without extracranial concomitant injuries are warranted to discern effectiveness and efficiency qualities of GTCS intervention.

Improved ultrasonographic 24-hour fluid requirement prediction - the IVC-shock index score. Fay Doucet ${ }^{1}$, Paula Ferrada $^{2}$, Sarab Murthi ${ }^{3}$, Ram Nirula ${ }^{4}$, Sara Edwards ${ }^{1}$, Emily Cantrell', Finfeng Han', Daniel Haase ${ }^{3}$, Andrew Singleton ${ }^{4}$, Yekaterina Birkas ${ }^{4}$, Giovanna Casola ${ }^{1}$, Raul Coimbra ${ }^{5}$. From the ${ }^{1}$ UC San Diego, San Diego, CA; ${ }^{2}$ Virginia Commonwealth University, Richmond, VA; the ${ }^{3}$ University of Maryland School of Medicine, R Adams Cowley Shock Trauma Center, Baltimore, MD; the ${ }^{4}$ University of Utah, Salt Lake City, UT; and the ${ }^{5}$ Riverside University Health System/ Loma Linda University, Loma Linda, CA.

Background: Identification of occult hypovolemia in trauma patients at admission is challenging. We previously showed response of ultrasonographic (US) respiratory minimum inferior vena cava size (IVCDMIN) or IVC Collapsibility Index (IVCCI) in repeated US examinations after 1 hour of IV fluid resuscitation is moderately predictive of 24-hour resuscitation fluid requirements (24FR). We hypothesize that combination of IVCDMIN or IVCCI with age, systolic blood pressure (BP), Shock Index (SI), or Revised Trauma Score improves ability to predict 24FR. Methods: Data from FAST-IVC, a prospective cohort trial at 4 level I trauma centers was used. Trauma patients were screened for an IVCDMIN of $12 \mathrm{~mm}$ or IVCCI of $50 \%$. A total of 196 patients were enrolled. IVCDMIN/IVCCI was obtained after 40-60 minutes of resuscitation. Regression was used to identify predictors of $24 \mathrm{FR}$ to be used as covariates in models with IVCDMIN/IVCCI to be used in receiver operating characteristic (ROC) analysis. A 24FR predictive score was created. Results: Combined models using ultrasonic assessment of the IVC and SI after 40-60 minutes of resuscitation improved the C-index for IVCDMIN from 0.74 (95\% confidence interval [CI] $0.65-0.84, p<0.0001)$ to 0.79 (95\% CI $0.71-0.88, p<$ $0.0001)$, and the C-index for IVCCI improved from $0.76(95 \%$ CI $0.66-0.86, p<0.0001)$ to 0.80 (95\% CI $0.71-0.88, p<$ $0.0001)$. A simplified IVC-SI score based on regression coefficients, using $0-3$ points for IVCDMIN and $0-5$ points for SI had a C-index of 0.78 (95\% CI 0.69-0.86, $p<0.0001)$ using the FAST-IVC data set. Conclusion: For major trauma patients, a regression model combining post-resus SI and IVCDMIN or IVCCI improves predictive ability for $24 \mathrm{FR}$ from good to strong. A simplified post-resus IVC-SI score was created; however, this score will require clinical validation.

Rotational thromboelastometry predicts mortality and disability in pediatric trauma. Aaron Cunningham ${ }^{1}$, Mary Condron $^{2}$, Martin Schreiber ${ }^{1}$, Kenneth Azarow ${ }^{1}$, Nicholas Hamilton $^{1}$, William Long ${ }^{3}$, Bryan Maxwell', Mubeen Fafri ${ }^{1}$. From the ${ }^{1}$ Oregon Health \& Science University, Portland,
OR; ${ }^{2}$ St. Charles Health System, Oregon; and the ${ }^{3}$ Legacy Emanuel Medical Center, Portland, OR.

Background: Traumatic coagulopathy seen on rotational thromboelastometry (ROTEM) is associated with poor outcomes in adults; however, this relationship is poorly understood in the pediatric population. We sought to evaluate the prognostic efficacy of ROTEM in injured children. Methods: Demographic and clinical outcomes data from severely injured children (age $<18 \mathrm{yr}$ ) admitted to a level I trauma centre, with a ROTEM, between 2014 and 2018, were retrospectively analyzed. Conventional coagulation parameters were compared with ROTEM. Wilcoxon rank-sum, Fisher exact, Pearson correlation and Youden statistics were used to evaluate the results. Results: Ninety-eight patients were reviewed. Median age in our population was 11 (interquartile range $[\mathrm{IQR}] 4-15$ ) years with a median Injury Severity Score of 17 (IQR 9-27). Increased EXTEM (tissue factor-triggered extrinsic pathway) and INTEM (ellagic acid activated intrinsic pathway) clotting time (CT; 113/250 s v. $65 / 144 \mathrm{~s}, p<0.001)$ and decreased EXTEM/INTEM maximal clot firmness (MCF; 45/45 mm v. 63/62 mm, $p<0.002$ ) were associated with mortality. EXTEM CT of 73.5 seconds or greater was predictive of mortality by Youden analysis. Prolonged EXTEM/INTEM CT (73/171 s v. 64/140 s, $p<0.002)$ were associated with disability $(n=32)$. In turn, EXTEM CT demonstrated strong correlation with international normalized ratio (INR; $r=0.625, p<0.001$ ). Traumatic brain injury (TBI; $n=58)$ was associated with increased EXTEM/INTEM CT $(69 / 155 \mathrm{~s} \mathrm{v} .64 / 140 \mathrm{~s}, p<0.02)$ in the presence of normal INR between groups (1.2 v. $1.1, p=0.09)$. Conclusion: Relative hypocoagulability on thromboelastometry correlates with conventional coagulation parameters and is associated with disability and mortality in children. Use of thromboelastometry can provide guidance for hemostatic resuscitation in pediatric trauma and may be more reliable in patients with TBI.

PSLS - Please somebody learn something! (Not just another 4-letter word...). Rita Clarke, Lisa Whitman, Heather Wilson, Heather Wong. From the Interior Health, Kelowna, BC.

Background: Trauma systems employ continuous measurement and evaluation of performance indicators (PIs) for performance improvement and patient safety (PIPS) purposes. The British Columbia Trauma Registry (BCTR) collects PIs retrospectively. Trauma nurse coordinators (TNCs) keep separate, prospectively collected, databases of locally defined PIs ("preregistry"). Interior Health (IH) TNCs sought to develop a system to support site-level PIPS in a standardized and secure database that could track, trend and report on quality issues in a timely fashion. Methods: A review of existing trauma PIPS databases in IH was performed. Subsequently, a standardized framework was developed, assigning PIs an $\alpha$-numeric value, and entering them into the Patient Safety Learning System (PSLS) database. Data are collected and entered concurrently, allowing real-time analysis of trauma trends, comparing all trauma patients whose care was reviewed by a TNC (denominator) in relation to patients whose care misses an identified PI (numerator). Results: The review of existing databases exposed challenges and inconsistencies with data collection within the current pre-registry database, most notably the inability to 
produce reports. TNCs consistently reported maintenance of informal databases, such as Excel spreadsheets, to monitor site PIPS. The advantage of these informal databases are ease of entry, flexibility, and site specificity. The disadvantages are that they are not secure, not reportable, and not easily accessible. $\mathrm{IH}$ TNCs in collaboration with the PSLS manger, successfully developed a user-friendly data collection and reporting system using the BC PSLS platform. This pre-existing quality framework provides comprehensive patient safety information to leaders and managers: it allows TNCs to instantly alert leaders to trauma issues at any site across IH. This novel use of the PSLS system has facilitated collection of standard PIs at both BCTR-supported sites, easily produced reports, maintenance of privacy and confidentiality standards, identification of trauma system trends, information-sharing across $\mathrm{IH}$, possible expansion to a provincial platform, and loop closure with referring regional and rural sites that do not have TNC or BCTR support. Conclusion: The IH regional trauma system PIPS program requires support from the BCTR and a reliable method of concurrent data collection. Previous data collection methods posed challenges with consistency, security, reportability, and accessibility. Using the BC PSLS to monitor trauma PIPS allows for trends and issues to be easily identified and reported. The BC PSLS maintains privacy and confidentiality standards, and allows for information sharing across IH and, potentially, province-wide.

Development of a personalized mortality prediction model for patients presenting with rib fractures. Tiffany Paradis, Jeremy Grushka, Tarek Razek, Paola Fata, Dan Deckelbaum, Kosar Kawaja, Andrew Beckett. From McGill University, Montreal, QC.

Background: Rib fractures are common injuries among trauma patients and can occur in the context of blunt trauma in addition to isolated injuries. As a result, these injuries are associated with increased morbidity and mortality, especially among the elderly. This has been demonstrated through prolonged hospital stay, ventilator requirements, and infectious complications. Our objective is to develop a personalized manner of predicting mortality in patients presenting with rib fractures at our level I trauma centre. Methods: Patients presenting with rib fractures between 1996 and 2016 were identified using Abbreviated Injury Scale (AIS) codes. A number of parameters, including age, gender, and Injury Severity Score (ISS), were selected and used to develop our prediction model. A multivariate logistic regression was performed using these parameters and incorporated into our dashboard calculator using R Shiny software. The calculator then returns a mortality risk based on the variables entered. Results: Our analysis identified chest wall AIS, ISS and age as predictors for patient mortality. Through the use R Shiny dashboard individual mortality risk estimates can be performed based on our hospitals' 20-year performance. Conclusion: Accessible personalized risk-prediction methods, based on hospital performance, will become increasingly important. Large data sets have the potential to identify individual patient risk specific to institutions and contribute to the personalization of trauma care. Although our model requires further validation, it is the initial step toward individualizing trauma care within our institution and future integration with artificial intelligence.
Changing demographics and mechanisms in rib fractures over 20 years at a Canadian level I trauma centre. Ian Beamish ${ }^{1}$, Tarek Razek ${ }^{1}$, Dan Deckelbaum ${ }^{1}$, Jeremy Grushka ${ }^{1}$, Paola Fata ${ }^{2}$, Andrew Beckett ${ }^{1,2}$. From ${ }^{1}$ McGill University, Montreal, QC; and the ${ }^{2} \mathrm{McGill}$ University Health Center, Montreal, QC.

Background: Rib fractures are one the most common findings in the trauma patient, in isolation or in combination with other injuries. Most rib fractures are due to blunt force from a variety of mechanisms. They are also associated with increased rates of pneumonia, mechanical ventilation and mortality. We wished to review our institution's 20-year experience with rib fractures to elucidate changing epidemiology and outcomes over time. Methods: The Montreal General Hospital's (MGH) trauma database was reviewed for the period 1996-2016. We used Abbreviated Injury Scale (AIS [CD1]) codes to identify all patients with rib fractures, extracting demographic and outcome data. ICD-9 codes were used for 1998-2005 and ICD-10 codes were used for 2006-2016 to classify patients into categories of mechanism of injury. Means and standard deviations for continuous variables and proportions for categorical variables were calculated overall and by age group. Results: The number of patients admitted at MGH with rib fractures is increasing over time, with 350 admissions in 2016. Most of the increase occurred in patients older than 65 years, where these patients outpaced those of other age groups, accounting for one-third of all patients. Rib fractures from ground-level falls have surpassed those from motor vehicle collisions (25.7\% v. $23.8 \%)$. Intensive care unit admissions, pneumonia events and mortality decreased across time, even in geriatric patients. Conclusion: These results have important implications for our trauma centre and will be used for planning for future geriatric trauma care requirements. The demographic shift of an aging population is now appearing in the trauma patient population. We demonstrated a "crossover point" in 2011 where more patients older than 65 years are being admitted with rib fractures than younger patients. Improved imaging techniques likely contributed partly to the increased prevalence of rib fractures.

The arrival of ride sharing in southwestern Ontario did not reduce the proportion of injuries resulting from alcohol related motor vehicle crashes. Rich Hilsden ${ }^{1}$, Mattbew Lund, Rob Leeper ${ }^{1,2}$. From ${ }^{1}$ Western University, London, $\mathrm{ON}$; and the ${ }^{2}$ London Health Sciences Centre, London, ON.

Background: Ride sharing is a new technology that increases the number of transportation options to city residents. The arrival of ride sharing has been associated with a reduction in alcoholrelated car crashes in several US jurisdictions. Whether ride sharing is associated with a reduction in alcohol-related car crashes in Canada has not been evaluated. Methods: A retrospective cohort study was performed using the South Western Ontario Regional Trauma Registry (SWORTR) between Apr. 1, 2013, and Mar. 31, 2018. Patients who were injured when ride sharing services were available constituted the ride sharing group and patients injured before the arrival of ride sharing in their region formed the control group. All patients with a positive alcohol level on arrival were considered to be involved in an alcoholrelated motor vehicle crash. Results: During the study period 
815 patients injured in motor vehicle crashes were entered in the SWORTR, and 690 patients had an alcohol level recorded. Of these, 123 patients had ride sharing available to them at the time of their collision and 567 did not. For the primary outcome, positive blood alcohol levels were seen in $23.6 \%$ of patients with access to ride sharing as compared with $17.6 \%$ of patients without ride sharing access $(p=0.126)$. Controlling for rural-urban differences by evaluating the city of London alone, there was still no significant difference observed in the proportion of patients with positive blood alcohol levels between those with and without access to ride sharing $(27.1 \%$ v. $17.0 \%, p=0.184)$. Evaluating drivers alone, no significant difference was observed in alcohol-related crashes with ride sharing services available compared with controls $(23.2 \% \mathrm{v}$. $17.8 \%, p=0.254)$. Evaluating drivers who crashed within the city of London alone, no difference was observed during the period where ride sharing was available compared with controls $(25 \% \mathrm{v}$. $18 \% p=0.348)$. A logistic regression analysis was performed with positive alcohol levels as the dependant variable. Among age, gender, and ride sharing availability, only male gender was independently associated with a positive alcohol level $(p=0.036)$. Conclusion: Alcohol-related motor vehicle collisions remain an important public health concern. Increased access to transportation options has been proposed to reduce the rates of such events. Our research failed to demonstrate any reduction in the proportion of injuries resulting from alcohol related motor vehicle crashes in southwestern Ontario with the arrival of ride sharing services.

Fresh whole blood use in trauma resuscitation: a descriptive systematic review on the effects on coagulopathy reversal and clinical outcomes. Igal Raizman', Lesley Gotlib Conn ${ }^{2}$, Rachel Strauss ${ }^{3}$, Barbara Haas ${ }^{3}$, Andrew Beckett ${ }^{4}$, Avery Natbens', Homer Tien', Feannie Callum ${ }^{3}$, Luis Teodoro $\mathrm{Da} \mathrm{Luz}^{3}$. From the ${ }^{1}$ University of Toronto, Toronto, ON; the ${ }^{2}$ Sunnybrook Research Institute, Toronto, ON; the ${ }^{3}$ Sunnybrook Health Sciences Centre, Toronto, ON; ${ }^{4}$ Mcgill University, Montreal, QC; and ${ }^{5}$ Ornge, Mississauga, ON.

Background: Massive hemorrhage is a major cause of potentially preventable deaths in trauma. Current literature suggests that transfusion of fresh whole blood (FWB) may be superior to component therapy in some clinical situations. However, level I evidence is lacking regarding its safety profile and efficacy in trauma. We conducted a systematic review on the use of FWB in trauma resuscitation and addressed its efficacy and safety on reversal of coagulopathy and other clinical outcomes, including mortality. Methods: Observational and experimental studies in humans were included. Medline, Embase and Cochrane were searched up to October 2018. Data on the effect of FWB on reversal of coagulopathy, clinical outcomes and safety were extracted. NewcastleOttawa and Cochrane Collaboration Risk of Bias Tool were used to assess risk of bias. The review was conducted in accordance with Preferred Reporting Items for Systematic Reviews and MetaAnalyses (PRISMA) guidelines and strength/quality of evidence was graded using Grading of Recommendations Assessment, Development and Evaluation (GRADE). Results: Fifteen studies met the inclusion criteria, consisting of 3 randomized controlled trials (RCTs), 8 retrospective cohort studies and 4 animal/ laboratory studies. Quality of the RCTs and cohort studies was low. Overall, the use of FWB was not associated with increased risk of mortality, and in some studies conferred a trend to survival advantage. The use of FWB was associated with improvement of trauma coagulopathy. Additionally, a trend toward increased risk of acute lung injury was also reported. Conclusion: The use of FWB in resuscitation of bleeding trauma patients is supported by lowquality studies that report association with improvement of trauma coagulopathy. The evidence is not robust to demonstrate superiority compared with component transfusion therapy, use of concentrate of clotting factors (e.g., prothrombin complex concentrate), or goal-directed therapy. Properly controlled experimental studies are warranted.

Early mobilization of trauma patients admitted to intensive care units: a systematic review and meta-analysis. Robert Green $^{1}$, Sean Higgins ${ }^{2}$, fenna Coles ${ }^{2}$, Mete Erdogan ${ }^{1}$. From ${ }^{1}$ Trauma Nova Scotia, Halifax, NS; and ${ }^{2}$ Dalhousie University Medical School, Halifax, NS.

Background: Previous systematic reviews suggest early mobilization in the general intensive care unit (ICU) population is feasible, safe, and may improve outcomes. Only 1 such review investigated mobilization specifically in trauma ICU patients and failed to identify any relevant articles. The objective of the present systematic review was to conduct an up-to-date search of the literature to assess the effect of early mobilization in adult trauma ICU patients on mortality, length of stay (LOS) and duration of mechanical ventilation. Methods: We performed a systematic search of 4 electronic databases (Ovid Medline, Embase, CINAHL, Cochrane Library) and the grey literature. To be included, studies must have compared early mobilization to delayed or no mobilization among trauma patients admitted to the ICU. Two authors independently extracted data using a standardized form. Meta-analysis was performed to determine the effect of early mobilization on mortality, hospital LOS, ICU LOS, and requirement for mechanical ventilation in days. Results: The search yielded 2975 records from the 4 databases and 7 records from grey literature and bibliographic searches; of these, 9 articles met all eligibility criteria and were included in the analysis. There were 7 studies performed in the United States, 1 study from China and 1 study from Norway. Study populations included neurotrauma (3 studies), blunt abdominal trauma (2 studies), mixed injury types (2 studies) and burns (1 study). Cohorts ranged in size from 15 to 1132 patients (median 63) and varied in inclusion criteria. Most studies used some form of stepwise progressive mobility protocol. Two studies used simple ambulation as the mobilization measure, and 1 study employed upright sitting as their only intervention. Time to commencement of the intervention was variable across studies, and only 2 studies specified the timing of mobilization initiation. We did not detect a difference in mortality with early mobilization, although the pooled risk ratio (RR) was reduced (RR 0.90 , 95\% confidence interval [CI] 0.74 to 1.09 ). Hospital LOS and ICU LOS were decreased with early mobilization, though this difference did not reach significance. Duration of mechanical ventilation was significantly shorter in the early mobilization group (mean difference -1.18 days, $95 \%$ CI -2.17 to -0.19 ). Conclusion: Our review identified few studies that examined mobilization of critically ill trauma patients in the ICU. On meta-analysis, early mobilization was found to reduce duration of mechanical ventilation, but the effects on mortality and LOS were not significant. Large controlled trials of higher quality are required to better define the potential of early mobilization of critically ill trauma patients. 
Impact of an early mobilization protocol on outcomes in trauma patients admitted to the intensive care unit: a retrospective cohort study. Robert Green ${ }^{1}$, Jenna Coles $^{2}$, Sean Higgins $^{2}$, Mete Erdogan' ${ }^{1}$ From ${ }^{1}$ Trauma Nova Scotia, Halifax, NS; and ${ }^{2}$ Dalhousie University Medical School, Halifax, NS.

Background: Long-term immobility has detrimental effects for critically ill patients admitted to the intensive care unit (ICU), including ICU-acquired weakness. Early mobilization of patients admitted to ICU has been demonstrated to be a safe, feasible and effective strategy to improve patient outcomes. The optimal mobilization of trauma ICU patients has not been extensively studied. Our objective was to determine the impact of an early mobilization protocol on outcomes among trauma patients admitted to the ICU. Methods: We analyzed all adult trauma patients (age $>18 \mathrm{yr}$ ) admitted to the ICU over a 2-year period before and following implementation of an early mobilization protocol, allowing for a 1-year transition period. Data were collected from the Nova Scotia Trauma Registry. We compared patient characteristics and outcomes (mortality, length of stay [LOS], ventilator days) between the pre- and postimplementation groups. Associations between early mobilization and clinical outcomes were estimated using binary and linear regression models. Results: Overall, there were 526 patients included in the analysis (292 preimplementation, 234 postimplementation). The study population ranged in age from 18 to 92 years (mean age $49.0 \pm 20.4 \mathrm{yr}$ ), and $74.3 \%$ of all patients were male. The pre- and postimplementation groups were similar in age, sex, and injury severity. In-hospital mortality was reduced in the postimplementation group $(25.3 \% \mathrm{v} .17 .5 \%, p=0.031)$. In addition, there was a reduction in ICU mortality in the postimplementation group (21.6\% v. $12.8 \%, p=0.009)$. We did not observe any difference in overall hospital LOS, ICU LOS, or ventilator days between the 2 groups. Compared with the preimplementation period, trauma patients admitted to the ICU following protocol implementation were less likely to die in hospital (odds ratio [OR] $0.52,95 \%$ confidence interval [C] $0.30-0.91, p=0.021)$ or in the ICU (OR $0.40,95 \%$ CI $0.21-0.76, p=0.005$ ). Results were similar following a sensitivity analysis limited to patients with blunt or penetrating injuries. There was no difference between the pre- and postimplementation groups with respect to in-hospital LOS, ICU LOS, or the number of ventilator days. Conclusion: This study represents one of the largest investigations to date on the impact of a structured progressive mobility protocol on outcomes in the critically ill trauma population. We found that trauma patients admitted to ICU during the postimplementation period had decreased odds of in-hospital mortality and ICU mortality. Ours is the first study to demonstrate a significant reduction in trauma mortality following implementation of an ICU mobility protocol.

Association between hypotension and mortality in critically ill patients with severe traumatic brain injury: experience at a single Canadian trauma centre. Robert Green ${ }^{1}$, Mete Erdogan $^{1}$, Nelofar Kureshi ${ }^{2}$, Lynne Fenerty ${ }^{2}$, Ginette ThibaultHalman $^{2}$, Simon Walling ${ }^{2}$, David B. Clarke. From 'Trauma Nova Scotia, Halifax, NS; the ${ }^{2}$ QEII Health Sciences Centre, Halifax, NS; and ${ }^{3}$ Dalhousie University, Halifax, NS.

Background: Hypotension is known to be associated with increased mortality in traumatic brain injury (TBI) patients. A systolic blood pressure (SBP) $<90 \mathrm{~mm} \mathrm{Hg}$ is the threshold for hypo- tension in consensus TBI treatment guidelines; however, some evidence suggests hypotension should be defined at higher levels for these patients. Our objective was to determine the influence of hypotension on mortality in severe TBI patients requiring intensive care unit (ICU) admission using different thresholds of SBP on arrival at the emergency department (ED). Methods: We conducted a retrospective cohort study of severe TBI patients (Abbreviated Injury Scale-Head score $\geq 3$ ) admitted to ICU at the QEII Health Sciences Centre (Halifax, Canada) between 2002 and 2013. Patients were grouped by SBP on ED arrival $(<90 \mathrm{~mm} \mathrm{Hg}$, $<100 \mathrm{~mm} \mathrm{Hg},<110 \mathrm{~mm} \mathrm{Hg}$ ). We performed multiple logistic regression analysis with mortality as the dependent variable. Models were adjusted for confounders including age, gender, Injury Severity Score (ISS), injury mechanism, and trauma team activation (TTA). Results: A total of 1233 patients sustained a severe TBI and were admitted to the ICU during the study period. The mean age was $43.4 \pm 23.9$ years, and most patients were male $(919 / 1233,74.5 \%)$. The most common mechanism of injury was motor vehicle collision $(491 / 1233,41.2 \%)$, followed by falls $(427 / 1233,35.8 \%)$. Mean length of stay in the ICU was $6.1 \pm$ 6.4 days, and the overall mortality rate was $22.7 \%$. SBP on arrival was available for 1182 patients. The $<90 \mathrm{~mm} \mathrm{Hg}$ group had $4.6 \%$ (54/1182) of these patients; mean ISS was $20.6 \pm 7.8$ and mortality was $40.7 \%$ (22/54). The < $100 \mathrm{~mm} \mathrm{Hg}$ group had 9.3\% (110/1182) of patients; mean ISS was $19.3 \pm 7.9$ and mortality was $34.5 \%$ (38/110). The < $110 \mathrm{~mm} \mathrm{Hg}$ group had 16.8\% (198/1182) of patients; mean ISS was $17.9 \pm 8.0$ and mortality was $28.8 \%$ (57/198). After adjusting for confounders, the association between hypotension and mortality was 2.22 (95\% confidence interval [CI] 1.19-4.16) using a $<90 \mathrm{~mm} \mathrm{Hg}$ cutoff, 1.79 (95\% CI 1.12-2.86) using a $<100 \mathrm{~mm} \mathrm{Hg}$ cutoff, and 1.50 (95\% CI 1.02-2.21) using a $<110 \mathrm{~mm} \mathrm{Hg}$ cutoff. Conclusion: While we found that TBI patients with $\mathrm{SBP}<90 \mathrm{~mm} \mathrm{Hg}$ were more than twice as likely to die, patients with $\mathrm{SBP}<110 \mathrm{~mm} \mathrm{Hg}$ on $\mathrm{ED}$ arrival were still 1.5 times more likely to die from their injuries as patients without hypotension. These results suggest that establishing a higher threshold for clinically meaningful hypotension in TBI patients is warranted.

How valid are our data? Establishing a minimum data validation process. Rosmin Esmail ${ }^{1}$, Christine Vis ${ }^{1}$, Sara Noswortby ${ }^{2}$. From ${ }^{1}$ Alberta Health Services, Calgary, AB; and the ${ }^{2}$ Foothills Medical Centre, Calgary, AB.

Background: In an effort to ensure that the data collected for the Alberta Trauma Registry (ATR) are accurate, valid and reliable, we reviewed the process of how the data are being validated in the ATR. Data validation practices vary and are inconsistent across the 10 level I-III trauma centres in the province. We determined that a minimum data validation process is vital to ensure validity and reliability of the data that entered into the ATR. Methods: A 6-step data validation process was created: 1) use the check and vendor validate function within the ATR; 2) for sites that submit to the Trauma Quality Improvement Program (TQIP; 3 out of 10 sites), use the vendor validate button; 3 ) data validation checks on 20 elements determined as critical to validate each month; 4) additional validation processes developed, as necessary; 5) trauma coordinator chart validation on clinically relevant elements for $5 \%$ of charts each year; and 6) by the 15 th of each quarter, report on the status of data abstraction and data validation. Results: 
This process was implemented as of May 8, 2018. Each data analyst is responsible for completing steps $1-4$ and 6 . This process empowers the data analysts to make corrections at the time of data entry/chart completion rather than retrospectively. Each quarter, the status of the data abstraction and chart validation is reported to the epidemiologist. Step 4 of the process, the trauma coordinator chart validation, was piloted in July 2018. The data elements from the chart included patient arrival date/time, patient discharge date/time, cause of injury, trauma team activation, complications, comorbidities, unplanned readmission to the intensive care unit and where the patient was discharged to, were compared with the registry data. Each trauma coordinator reviewed 2 charts during the pilot. The number of errors ranged from 0 to 10 per chart. Time to review the charts ranged from 1 minute to 2 hours. The types of errors found were in the dates, complications and comorbidities. Any discrepancies were corrected with the data analysts. Comments from the pilot were incorporated into the data validation spreadsheet. Step 5 of the validation process was implemented as of September 2018. Challenges in the process include getting buy-in and support, the time required to conduct validation checks and chart validation (any chart validation is dependent on the chart abstraction), and ensuring user friendliness of the chart validation tool. Conclusion: Establishing a minimum data validation process will ensure the data in the ATR is accurate and reliable. It is also an essential part of best practices in data integrity. This is a novel undertaking as, to our knowledge, no other Canadian trauma centre or system has established such a process. This process provides data validation best practices that can be used by other trauma centres.

Trauma in northern Quebec. Feongyoon (fenny) Moon ${ }^{1}$, Tarek Razek ${ }^{2}$, Natbalie Boulanger ${ }^{3}$, Dan Deckelbaum ${ }^{2}$, Feremy Grushka ${ }^{2}$, Paola Fata ${ }^{1}$, Andrew Beckett ${ }^{1}$, Kosar Kbwaja ${ }^{1}$. From the ${ }^{1}$ McGill University Health Centre, Montreal, QC; ${ }^{2}$ McGill University, Montreal, QC; and the ${ }^{3}$ Centre de Santé Tulattavik de l'Ungava, Kuujjuaq, QC.

Background: Trauma care in northern Quebec is fragmented and lacks resources, despite covering a large area with rugged geography and climate. Nunavik makes up the northern third of Quebec. The Inuit people residing in Nunavik are vulnerable to more frequent and severe trauma injuries owing to environmental and social factors. Compared to the rest of the Canadian population, the Indigenous people of Canada experience lower life expectancy and higher rates of substance abuse, suicide, and addiction, which collectively contribute to trauma epidemiology. Methods: Retrospective data collection was performed on all trauma patients admitted to Kuujjuaq's Centre de Santé Tulattavik de l'Ungava (CSTU) from 2005 to 2014. For the 797 trauma cases identified during the specified time period, demographic and health services data, including mechanism of injury, transfer times, prehospital and emergency vitals measurement, and patient outcomes were extracted. These data were studied in conjunction with the coroner's reports on trauma mortality occurring in Nunavik. Results: Motor vehicle crashes (MVCs; 32.4\%), falls (17.2\%), intoxication (12.9\%), and blunt assault (11.9\%) were the most common causes of injury. For $46.5 \%$ of all injuries, patients were found to be alcohol-intoxicated on arrival to the emergence department (ED). Of 229 MVCs, 151 cases had documentation of helmet and seat- belt use at time of accident: 145 (96.0\%) had no helmet on and $164(71.2 \%)$ were not wearing seatbelts. Most (64.5\%) patients were admitted to CSTU from the ED. About one-third $32.9 \%$, $n=262$ ) of patients were transferred to a tertiary care centre in Montreal, either directly from the ED or following a short admission. Out of 262 transferred trauma patients, 122 (46.6\%) were transferred for computed tomography (CT) imaging alone. The next most common indications for transfer were fracture management $(n=82)$ and airway management $(n=20)$. Of the 122 patients who were transferred only for CT imaging, 104 $(85.2 \%)$ patients met the indication for CT imaging according to the Canadian head CT and C-spine rule or most recent guidelines for imaging of other body regions. Of all patients transferred and admitted to McGill's tertiary care centre, $57.9 \%$ underwent surgical intervention, $27.8 \%$ had a medical intervention, and $14.3 \%$ stayed for observation. The average wait time for transfer from Kuujjuaq to Montreal was 7.7 hours. The Office of the Coroner of Quebec identified 295 trauma deaths in Nunavik during the study period. The leading cause of death was self-inflicted injury by hanging, strangulation, and suffocation (34.6\%), followed by MVC (7.1\%) and exposure to excessive cold. Conclusion: To our knowledge, this is the first study to characterize trauma in northern Quebec. Our study allowed for better characterization of the burden of injury, transport within Nunavik and to Montreal, the hidden trauma mortality, and opportunities for injury prevention and optimization of trauma systems in this unique setting. By undertaking this study, we were able to establish new partnership between leadership in northern Quebec and the trauma team at McGill University Health Centre.

Contemporary trends and outcomes of traumatic colon injuries requiring resection. Vincent Cheng, Morgan Schellenberg, Kenji Inaba, Zachary Warriner, Marc D. Trust, Kazubide Matsushima, Lydia Lam, Demetrios Demetriades. From the LAC+USC Medical Center, Los Angeles, CA.

Background: Following traumatic injury, the frequency of primary anastomosis after colon resection has overtaken that of ostomy diversion. This change may be driven in part by improved technology facilitating primary anastomosis speed and integrity. Trends in ostomy versus anastomosis have yet to be quantified, and recent literature comparing clinical outcomes is incomplete. The purpose of this study was to quantify contemporary trends in surgical management after colon resection for trauma and to compare clinical outcomes. Methods: The National Trauma Databank (2007-2014) was queried for all colon injuries requiring resection. Procedure codes were used to define study groups as patients undergoing either ostomy or anastomosis following resection. The proportion of patients undergoing ostomy was compared over time. Multivariate regression analyses adjusted for differences in patient baseline characteristics and evaluated the impact of ostomy versus anastomosis on mortality, hospital length of hospital stay (LOS), intensive care unit (ICU) LOS, and ventilator days. Results: A total of 13806 colon injuries requiring colon resection were identified. Of these patients, $3823(27.7 \%)$ underwent ostomy creation and 9983 (72.3\%) underwent anastomosis. Ostomy frequency varied by year: $27.9 \%$ in 2007 , rising to $29.1 \%$ in 2011 , and falling to $26.6 \%$ in 2014 . Of all patients, $3809(27.7 \%)$ were male, and the median age was 30 years (interquartile range 22-43). A total of 
4201 patients (30.4\%) and 9428 patients (68.3\%) sustained blunt and penetrating injuries, respectively. The 2 most common mechanisms were gunshot wound (8743 patients, $63.3 \%)$ and motor vehicle collision (3322 patients, 24.1\%). The descending colon was the most commonly injured segment (3625 patients, $26.3 \%$ ), followed by sigmoid (3029 patients, $21.9 \%$ ), ascending (2456 patients, $17.6 \%$ ), and transverse (1636 patients, $11.8 \%$ ); 3623 patients (26.2\%) had unspecified colon injuries. After controlling for differences in patient baseline characteristics, ostomy was significantly associated with lower rates of mortality in all colon injuries (odds ratio [OR] $0.354, p<0.001$ ), ascending injuries (OR 0.439, $p=0.001)$, transverse injuries (OR 0.353, $p<$ 0.001 ), descending injuries (OR $0.412, p<0.001$ ), and sigmoid injuries (OR $0.284, p<0.001)$. Overall, ostomy was also significantly associated with longer hospital LOS (regression coefficient [RC] $0.586, p<0.001)$, ICU LOS (RC 0.415, $p<0.001$ ) and ventilator days (RC $0.419, p<0.001$ ). Conclusion: Even after adjusting for patient baseline characteristics, ostomy creation following resection for trauma was significantly associated with lower rates of mortality regardless of colon injury location. The increased hospital LOS, ICU LOS, and ventilator days associated with ostomy creation may therefore reflect a survival bias. Despite the recent downtrend in ostomy frequency, these results suggest that ostomy creation may represent a favourable approach to minimize mortality risk following colonic resection for traumatic injury.

Effective communication during patient transitions. Angie Brisson $^{1}$, Nasira Lakba ${ }^{2}$, Heather Wong ${ }^{3}$, Laurie McLauchlin ${ }^{2}$, Caleigh Sullivan Ashe ${ }^{1}$, Suzie Allen Logie ${ }^{2}$. From ${ }^{1}$ Vancouver Coastal Health, Vancouver, BC; the ${ }^{2}$ Vancouver General Hospital, Vancouver, BC; and ${ }^{3}$ Interior Health, Kelowna, BC.

Background: Effective communication is an integral part of patient safety, especially during transition points, as patients move within the health care system. Previously, trauma patients were transferred from high acuity (HA) to a trauma ward, with no standardized written handover tool for reporting, relying on verbal nurse-to-nurse reporting. This resulted in inconsistencies in information being communicated, impacting patient care and safety. A standardized tool may reduce communication errors and missed information. Methods: A qualitative survey was conducted on the HA and trauma wards over a 4-week period to determine the effectiveness of the handover process before and after the implementation of a standardized tool. This survey was distributed to all staff and used to determine the perception of readiness to care for patients post-transfer, evaluating the consistency, completeness, and accuracy of information relayed and received. Nurses on both units were observed during handover at various times. Results: Sixty percent of nurses from both units working during the 4-week period responded to the survey. Forty percent of respondents had less than 1 year of experience. Most perceived receiving adequate information during handover; however, gaps were identified, including omission of information regarding medications, diagnostics, trajectory of care in $\mathrm{HA}$, and care needs. Other inconsistencies included variation in verbal reporting, misinterpretation of patient status before transfer, varying interpretation of patient assessment, and miscommunication regarding transfer orders. What was important to one nurse was not deemed important to another. The introduction of a standardized, systemized handover tool followed by a verbal report helped clarify questions and alleviate any inconsistencies, better informing and preparing ward nurses caring for the patients. Nurses identified a systematic approach and reference to pertinent information as essential features of effective handover, including patient details, presenting problem, treatment, future care/disposition plan, and nursing observations. The tool allowed for a common dialogue between nurses and helped alleviate any miscommunication, repeat phone calls, and reduced time spent looking for information in the chart. The tool provided a guide for the novice nurse to assess and report using a systematic format at daily trauma rounds. Ten percent of the nurses felt this was an extra step to the transfer process taking more time, but recognized the importance of relaying consistent information for ongoing patient care and safety. Conclusion: Overall, these results demonstrate that standardized handover protocols and reporting improve provider readiness in caring for complex trauma patients. This process allows for a seamless and safe process of communication.

Safe reduction of abdominal CT imaging in pediatric trauma patients: a quality-improvement initiative. Suzanne Beno, Talia Lenton-Brym, Daniel Rosenfield, Dorotby McDowall, Paul Wales, Tania Principi. From the Hospital for Sick Children, Toronto, ON.

Background: Studies indicate $17 \%-25 \%$ of children receiving abdominal computed tomography (CT) imaging after trauma are at very low risk for intraabdominal injury (IAI), with expected positive results of $0.1 \%$. Unnecessary imaging in this setting exposes children to ionizing radiation, procedural sedation, and increases cost to the health care system. We aimed to reduce abdominal CT imaging in pediatric trauma patients at very low risk for IAI by $20 \%$ over an 8 -month timeframe. Methods: Chart review, followed by prospective time series using the Model for Improvement, was performed for patients receiving abdominal CT imaging at a pediatric trauma centre between April 2016 and September 2018. The primary outcome was proportion of abdominal CT scans in patients at very low risk for IAI. CT scans ordered within 24 hours of admission, return visits to the emergency department (ED) within 72 hours and hospital admission with newly diagnosed IAI were collected as balancing measures to evaluate for missed injuries. Results: A chart review audit revealed $183 / 359(51.0 \%)$ of pediatric trauma patients between April 2016 and December 2017 had abdominal CT scans obtained, of whom 49/183 (26.8\%) were considered to be at very low risk for IAI, as defined by published clinical decision rules. A quality-improvement initiative ensued that included a multifaceted collaborative approach of education, audit and feedback, and system/process-based interventions with 7 plan-dostudy-act (PDSA) cycles. Interventions included 1) trauma diagnostic imaging algorithm ratification, 2) multidisciplinary trauma provider education, 3) project engagement as a hospital Choosing Wisely initiative, 4) forced-option dedicated trauma CT requisition development and dissemination, and 5) audit and feedback cycles of all trauma patients for whom abdominal CT scans were obtained. Post-intervention, the proportion of abdominal CT scans in all trauma patients $(60 / 178)$ and those at very low risk for IAI (6/60) significantly decreased over time. Statistical process 
control revealed a statistically significant change, with an absolute reduction in the proportion of abdominal CT scans of $17.3 \%(p=$ $0.000)$ in all trauma patients and $16.8 \%(p=0.007)$ in patients at very low risk for IAI. No missed intra-abdominal injuries occurred, as measured by positive abdominal CT scans within 24 hours of admission, return visits to an ED, or hospital admissions with newly diagnosed IAI. Conclusion: This qualityimprovement initiative using root-cause analysis, PDSA cycles and statistical process control to measure the effect of a dedicated institutional trauma diagnostic imaging algorithm and forcedoption trauma CT requisition for pediatric abdominal CT imaging resulted in a $17 \%$ reduction in abdominal CT imaging in those at very low risk for IAI, with no missed injuries. This initiative safely and significantly reduced unnecessary testing and ionizing radiation in a vulnerable population.

Autotransfusion of traumatic hemothorax revisited - a scoping review with focus on application in the austere environment. Christian Heck. From the University of British Columbia and Vancouver General Hospital Trauma Services, Vancouver BC; and Doctors Without Borders.

Background: Direct retransfusion of shed blood from a traumatic hemothorax - without the use of a cell saver device has been described in Western trauma centres and in the humanitarian or military settings. It is mentioned in recent Advanced Trauma Life Support (ATLS) guidelines, but the method seems not to be widely used. The prompt availability of blood products in academic trauma centres made autotransfusion less useful, but there is continuing interest to use it in the austere environment. Methods: English, French and German literature since 1997, including available national trauma guidelines and recommendations from military and humanitarian organizations, are analyzed for a scoping review. Own cases from deployments with a humanitarian organization and from a level I Canadian trauma centre are analyzed to describe the practicalities and potential of the method with a focus on the low resource environment. Results: A preliminary search using medical subject headings ("blood transfusion, autologous" OR "autotransfusion" AND "haemothorax" OR "hemothorax" AND "humans" AND English[lang], articles since 1997) identified only 1 medium-sized retrospective multicentre study and 5 case series, the latter mostly from a humanitarian or military environment. Two studies examined in vitro coagulation properties of shed hemothorax blood. None of the clinical case series reported complications directly related to the autotransfusion, but in vitro studies do raise concerns about the potential for inducing coagulopathy. The techniques to retransfuse blood from the chest cavity described in the published literature and guidelines vary between the use of commercial, purpose-built collection systems and improvised or adapted low-tech collecting systems that are used primarily in the humanitarian setting. No consensus exists concerning the safe time window since injury for autotransfusion of blood, the potential role of contamination in penetrating chest injuries, the effects on coagulation in the trauma victim or a minimum technical standard for the procedure. Conclusion: Direct autotransfusion of shed blood from the chest - without the use of a cellsaver device is a viable option for the exsanguinating patient in the prehospital, military or humanitarian environment. There are few data regarding autotransfusion, and there is still some controversy about its safety. However, especially in an austere or underresourced environment, autotransfusion can be life-saving. The method used by humanitarian organizations is simple and requires only minimal additional equipment.

Obesity is not protective against visceral injury from gunshot wounds. Emily Koeck ${ }^{1}$, Justin Mis ${ }^{1}$, Matt Kaminsky ${ }^{2}$, Faran Bokbari'. From the ${ }^{1}$ Cook County Hospital, Chicago, IL; and the ${ }^{2}$ Cook County Trauma Unit, Chicago IL.

Background: As the obesity epidemic expands across North America, so does the incidence of gunshot wounds in the obese population. Obesity has been studied as a protective factor in blunt trauma and has been shown to protect against visceral injury in stab wounds. The association between obesity and rate of visceral injury in gunshot wounds has not yet been studied, and there are limited data on outcomes after gunshot wounds in obese patients. Methods: We performed a retrospective review of prospectively collected data on abdominal gunshot victims who presented to an urban level I trauma centre between January 2016 and August 2018. Data collected included demographics, emergency department vitals and trauma variables, body mass index (BMI), computed tomography (CT) imaging, operative management, presence of visceral injury, and outcomes of mortality and complications. Patients were stratified into BMI groups $(<18.5$, $18.5-24.9,25-29.9,30-35,>35)$, with primary outcome of visceral injury. Results: Of 503 patients identified with abdominal gunshot wounds, 334 had a complete data set for analysis. Age increased across increasing BMI groups (18.7, 24.5, 26.3, 30.6, $30.8, p<0.001)$. There was no difference in Injury Severity Score (ISS), though there were significant differences in percentage of critically injured (ISS > 25) patients $(24.3 \%, 30.8 \%, 19.6 \%, 34 \%$, $27.3 \%, p=0.047)$. There were no differences in percentage of patients undergoing CT imaging $(61.5 \%, 58 \%, 67 \%, 52.1 \%$, $69.7 \%, p=0.32)$, likelihood of operation $(84.6 \%, 72.7 \%, 75.3 \%$, $79.2 \%, 93.9 \%, p=0.11$ ), or presence of visceral injury $(84.6 \%$, $63.6 \%, 72.2 \%, 68.8 \%, 75.7 \%, p=0.34)$. Nontherapeutic laparotomy was not statistically significant, though appeared to be increased in the 2 greatest BMI groups $(0 \%, 12.5 \%, 4.1 \%$, $13.2 \%, 19.4 \%, p=0.089)$. There were significant differences in hospital length of stay (LOS; 16.9, 10.5, 10.9, 14.3, 21.6, $p=$ $0.004)$; intensive care unit (ICU) LOS $(11.9,4.54,6.2,8.9,12.2$, $p=0.01)$, and ventilator days $(4.3,1.9,2.5,5.8,6.7, p=0.03)$ across BMI groups, with highest values at the extremes of BMI. Incidence of complications increased across BMI groups (15.4\%, $11.2 \%, 14.4 \%, 25 \%, 30.3 \%, p=0.03)$. There were no differences in in-hospital mortality $(0 \%, 5.6 \%, 9.3 \%, 10.4 \%, 9.1 \%, p=0.58)$. Conclusion: This study demonstrates that BMI is not protective against visceral injury in abdominal gunshot wounds. Among obese patients who suffer abdominal gunshot wounds, hospital and ICU LOS and ventilator days are increased, as is the incidence of complications. As the prevalence of obesity increases, its association with poor outcomes in gunshot wounds will require further investigation.

Extremity trauma results in severe coagulopathy and impaired fibrinolysis based on serial thromboelastography. Prism Schneider ${ }^{1}$,Elabeb Rabbar ${ }^{2}$, Bryan Cotton ${ }^{3}$. From the ${ }^{1}$ University of Calgary, Calgary, $\mathrm{AB} ;{ }^{2}$ Wake Forrest 
University, Winston-Salem, NC; and the ${ }^{3}$ University of Texas, Houston, TX.

Background: Trauma-induced coagulopathy (TIC) correlates with morbidity and mortality. Thromboelastography (TEG) evaluates the clotting function of whole blood, and elevated maximal amplitude (MA) has been shown to be predictive of in-hospital venous thromboembolic events (VTE). To date, coagulopathy using TEG has not been studied in major extremity trauma beyond 24 hours postinjury. We hypothesized that patients with major extremity trauma would continue to be hypercoagulable beyond 24 hours and would demonstrate increased VTE incidence. Methods: This was a subset analysis of a prospective, observational trial of level I trauma activations arriving within 6 hours of injury. Serial TEG and traditional coagulation testing were obtained at admission and at $3,6,12,24,48,72,96$, and 120 hours. VTEs were defined as deep vein thrombosis (DVT) or pulmonary embolism (PE) during hospitalization. Patients were dichotomized into ORTHO (extremity Abbreviated Injuruy Scale [AIS] score $>2$ ) or CONTROLS (extremity AIS score 0-2). Univariate analysis and multiple logistic regression was developed to evaluate VTE incidence. Results: A total of 795 patients were enrolled; of these, 340 had complete serial TEG data sets through 5 days postinjury. Patients were excluded if they were younger than 18 years of age, incarcerated, or had associated burns on more than $20 \%$ of the total body surface area. Seventy-five patients were classified as ORTHO and 265 were CONTROLS. There were no differences in baseline demographics or nonextremity AIS scores by body region. ORTHO patients were more likely to have a blunt mechanism of injury $(91 \%$ v. $62 \%)$ and to have higher overall Injury Severity Scores (median 27 v. 17, both $p<0.001$ ). ORTHO patients were more unstable on arrival (median Revised Trauma Score 3.51 v. $6.81, p=0.035$ ). Univariate analysis was conducted, followed by longitudinal analysis using generalized estimating equations (GEE) to evaluate group $\times$ time interactions on changes in TEG, controlling for age, sex, base deficit and group. A multiple logistic regression was developed to evaluate VTE incidence. Based on the GEE model, the ORTHO group was more hypocoagulable, as measured by k-time and decreased MA, over the first 5 days postinjury. However, despite being unexpectedly more hypocoagulable, the ORTHO patients demonstrated impaired lysis $(p<0.05)$ and had an increased VTE rate $(13.3 \%$ v. $1.8 \%, p<0.001)$. On multivariate analysis, ORTHO group was an independent predictor of in-hospital VTE (odds ratio 6.36, 95\% confidence interval 1.94-20.81, $p=0.002$ ). Conclusion: Based on 5-day serial TEG analysis of 340 trauma patients, the ORTHO group showed more profound coagulopathy. From 12 hours postinjury onwards, both groups become progressively more hypercoagulable; however, somewhat in contrast to our hypothesis, ORTHO patients were more hypocoagulable. Despite this, the ORTHO patients had decreased fibrinolysis, which may translate into higher VTE and warrants further investigation. Major extremity trauma also remains a strong, independent predictor of VTE.

Assessment of a standardized trauma panel in children presenting to a level I pediatric trauma centre. Chandandeep Bal $^{1}$, Paul Bryan ${ }^{2}$, Sherry MacGillivray ${ }^{3}$, Grabam Thompson ${ }^{4}$, Ian Wishart ${ }^{2}$. From the ${ }^{1}$ University of Toronto, Toronto, $\mathrm{ON}$; the ${ }^{2}$ University of Calgary, Calgary, AB;
${ }^{3}$ Alberta Health Services, Calgary, AB; and the ${ }^{4}$ Alberta Children's Hospital, Calgary, AB.

Background: Standardized trauma laboratory panels (TLP) are common among pediatric emergency centres. However, the utility of some investigations included in TLPs has been shown to be limited. This project aimed to determine adherence to a TLP at a level I pediatric trauma centre, where the trauma team activation (TTA) protocol mandates the full trauma panel be performed. Secondary objectives included determining the proportion of abnormal results for each test and describing associated interventions. Methods: Patients younger than 18 years old who had a TTA between October 2007 and November 2016 were identified using the trauma registry database. Demographics, mechanism of injury, Injury Severity Scores (ISS), investigations and emergent management were collected through a retrospective medical record review. Adherence to performing the complete TLP and completion of individual components was evaluated. The proportion of abnormal laboratory investigations from the panel and the proportion of investigations that resulted in specific interventions were calculated. Results: In total, 829 TTAs were identified. The mean ISS was $7.7 \pm 10.2$. Overall, adherence to performing the complete TLP was $2 \%(15 / 829)$. Most $(511,62 \%)$ children had at least 1 component of the TLP completed. The most common investigations ordered from the panel were complete blood count and serum electrolytes ( $76 \%$ and $70 \%$, respectively). Phosphate and ethanol level were omitted most frequently $(98 \%$ and $68 \%$, respectively). Overall 750/7078 (11\%) of all laboratory results were abnormal and 126/7078 (1.8\%) of all results led to an emergent intervention. The investigations associated with the highest rate of diagnostic intervention when results were outside the normal range were alanine aminotransferase $(91 / 129,71 \%)$ and lipase $(29 / 34,85 \%)$; a computed tomography scan of the abdomen was performed in 78/129 (61\%) and 23/34 (68\%) of abnormal values, respectively; 2 required surgical intervention. Potassium, international normalized ratio and calcium levels were most frequently abnormal (111/584 [19\%], 106/562 [19\%], 71/539 [13\%], respectively), but infrequently required any intervention $(2 / 111,4 / 106$, $1 / 71$, respectively). Creatinine, magnesium, glucose and sodium had the lowest proportion of abnormal values, (2/576 [0\%], 7/545 [1\%], 27/575 [4.7\%], 29/583 [5.0\%], respectively). None of these tests resulted in any intervention. Conclusion: Most pediatric emergency department patients who triggered a TTA did not have the full trauma laboratory panel completed. The proportion of abnormal results ranged widely across individual components in the panel. Only a small number of abnormal values resulted in emergent intervention. Further study is required to determine the optimal use of laboratory investigations in children presenting to a level I pediatric trauma centre following a traumatic injury.

The role of extracorporeal life support in management of severely injured trauma patients. Yi Man (David) Ko ${ }^{1}$, Morad Hameed ${ }^{2}$, Emilie Foos $^{2}$, David Evans ${ }^{2}$, Naisan Garraway $^{2}$, Philip Dawe ${ }^{2}$. From the ${ }^{1}$ University of British Columbia, Vancouver, $\mathrm{BC}$; and the ${ }^{2}$ Vancouver General Hospital, Vancouver, BC.

Background: Patients suffering from massive injuries often die from complications of hypovolemia, hypothermia and acidosis. Extracorporeal life support (ECLS) has the capacity to oxygenate 
and rewarm blood, correct hypercarbia, and provide circulatory support. However, the application of ECLS in trauma has been underutilized owing to the risk of worsening hemorrhage as a result of systemic anticoagulation. This study examined survival and complication rates in trauma patients undergoing ECLS at a level I trauma centre. Methods: Data from the BC Trauma Registry were retrospectively reviewed to identify trauma patients who received ECLS between 2001 and 2018 at Vancouver General Hospital (VGH). Demographic information, injury type, mechanism of injury and clinical outcomes were reviewed in these patients. Results: From 2001 to 2018, 14 adult trauma patients were identified and included in the study. Trauma patients were predominately male (71\%), with a mean age of $37.4 \pm 18$ (range 17-64) years. Thoracic injury $(n=$ 8 ) was the most common diagnosis followed by abdominal injury $(n=3)$. Mechanism of injury was primarily motor vehicle collision $(n=7)$. Half of ECLS was venoarterial in configuration for cardiac support and the remaining was in venovenous configuration. ECLS duration was $3 \pm 1.7$ days, with no systemic anticoagulation. The most common ECLS complication was bleeding at the cannula site $(n=2)$ followed by venous thromboembolism $(n=1)$. Overall survival from ECLS was $86 \%$, and survival to hospital discharge was $57 \%$ in the cohort. Multiple organ failure was the most common cause of death $(67 \%)$. Conclusion: With an improved safety profile, ECLS appears to be an effective treatment option in selected groups of trauma patients and is safe across a spectrum of injury patterns, including severe thoracic and abdominal trauma. In collaboration with the critical care group at VGH, we recently implemented an ECLS protocol to better identify these patients and streamline their care. We hope to study this cohort prospectively and inform future guidelines for ECLS in trauma.

Did change in alert criteria improve time to identification, treatment, and outcomes of warfarin-associated traumatic brain injury? Manuel Martinez, Jeffrey Wild, Kenneth Widom, Denise Torres, Joseph Blansfield, Mobsen Shababang, Fames Dove, Marcus Fluck. From the Geisinger Medical Center, Danville, PA.

Background: Traumatic brain injury (TBI) sustained by a patient on warfarin anticoagulation therapy can have substantial mortality - more so when compared with injured patients who are not on such therapy. The purpose of this study was to identify if the time to identification and early treatment of patients with mild TBI on warfarin anticoagulation therapy was improved with a trauma alert activation and the impact this would have on patient outcomes. Methods: All patients sustaining mild TBI in a level I trauma centre between Jan. 1, 2007, and Dec. 18, 2016, were retrospectively reviewed using the electronic medical record (EMR). The patients were separated into preimplementation and postimplementation groups. All patients had to have an elevated international normalized ratio on admission and had to be on coumadin. We used Fisher exact, $\chi^{2}$, and Wilcoxon signed-rank tests to identify significant differences between the groups. Results: Cohorts were composed of a preimplementation $(n=$ $35,55.5 \%)$ and a postimplementation $(n=28,44.5 \%)$ group. There was no statistically significant difference in the age, mechanism of injury, loss of consciousness or mean Glasgow Coma Scale score on admission. The timing difference from presenta- tion to imaging was not statistically significant between the preimplementation and postimplementation groups (98 min v. $94 \min , p=0.371$ ). However, the timing difference from imaging diagnosis to treatment was statistically significant between the preimplementation and postimplementation groups (144.5 min v. $52 \mathrm{~min}, p=0.003)$. The timing difference from admission to treatment was also statistically significant $(238 \mathrm{~min} \mathrm{v.} 148 \mathrm{~min}$, $p=0.028)$. Despite timing improvements in starting treatment for warfarin reversal, there was no statistically significant difference in length of stay, comfort/hospice status while admitted, or discharge status. Conclusion: Patients with mild TBI still have a high susceptibility to decompensate and still are at risk of high morbidity and mortality. The creation of a protocol-driven treatment algorithm has resulted in a more uniform care pattern and a heightened awareness of the consequences of an unidentified, and thus untreated TBI. This study also confirms that although time to reversal was improved, it did not have a significant impact in the patient's overall outcome.

Time-driven activity-based costing for acute trauma care. David Kim ${ }^{1}$, Morad Hameed ${ }^{1}$, Larissa Roux ${ }^{2}$, Andrew Nicol ${ }^{2}$. From the ${ }^{1}$ Vancouver General Hospital, Vancouver, BC; and the ${ }^{2}$ University of British Columbia, Vancouver, BC.

Background: Trauma is a leading cause of morbidity and mortality globally and places a heavy and as yet largely unquantified, financial burden on society. A micro-costing approach known as time-dependent activity-based costing (TDABC) has recently been proposed as a means to measure the financial burden of trauma care. This study describes an early experience with a real time approach to TDABC in a busy level I trauma centre. Methods: The Trauma Unit at Groote Schuur Hospital in Cape Town, South Africa, uses an electronic clinical documentation interface during resuscitation of trauma patients to create trauma care process maps that track and time stamp all resuscitative assessments and interventions, linking them with costs derived from North American actuarial. A study investigator shadowed the trauma team for a 14-day period, entering all aspects of trauma resuscitation into the platform in real time. Results: A total of 96 patients were documented. Of these, 77 were males and 19 were females. Mean age was 39 (range 15-96) years. Their trauma mechanisms were road traffic $(n=24)$, stab wound $(n=$ $21)$, gunshot wound $(n=17)$, fall $(n=14)$, community assault $(n=$ $9)$, struck against or by object $(n=5)$, or struck against or by person $(n=3)$. Process maps were developed for each patient and showed an average resuscitation time of 15 hours. Average resuscitation cost was calculated per patient $(\$ 847)$, which was categorized into equipment (\$37), space (\$726) and supplies (\$94). These average costs were calculated for high- (\$1526) and low$(\$ 725)$ acuity patients. The total resuscitation costs for the 96 patients was $\$ 79766$. Conclusion: It is feasible to apply principles of TDABC to the measurement of costs of acute trauma care. Digital technology allows this costing strategy to be applied to all patients in real time, although the accuracy of the method is still limited by our incomplete understanding of true costs of specific health care commodities. To our knowledge, this is the first application of real time TDABC in trauma care.

Damage control thoracic surgery allows for correction of acidosis after chest trauma: a 10-year experience with chest 
packing. Fobn Tierney ${ }^{1}$, Lilia Schulenberg ${ }^{2}$, Charles Fredericks ${ }^{2}$, Thomas Messer ${ }^{1}$, Fredric Starr ${ }^{3}$, Andrew Dennis ${ }^{3}$, Faran Bokbari ${ }^{1}$, Matt Kaminsky ${ }^{3}$. From the ${ }^{1}$ John $\mathbf{H}$. Stroger Jr. Hospital of Cook County, Chicago, IL; the ${ }^{2}$ Rush University Medical Center, Chicago, IL; and the ${ }^{3}$ Cook County Hostpital, Chicago, IL.

Background: Damage control principles are well defined for abdominal trauma, but their role remains unclear in thoracic trauma. We sought to characterize survival after damage control thoracic surgery and to determine whether acidosis is corrected after damage control packing. Methods: Patients who underwent damage control thoracotomy and/or median sternotomy with temporary closure and packing from 2008 to 2018 were identified. The primary outcome was 24-hour survival; secondary outcomes were change in base deficit before closure, complications, need for redo thoracotomy, and in-hospital mortality. Descriptive statistics, Student $t$ tests, and Fisher exact tests were used. Results: Sixty-eight patients were included, of whom 55 (80.1\%) sustained a gunshot wound. Fifty-seven patients $(83.8 \%)$ underwent thoracotomy, $6(8.8 \%)$ sternotomy, and $5(7.4 \%)$ both thoracotomy and sternotomy. Forty-one patients $(60.3 \%)$ survived 24 hours. Nonsurvivors were more likely to undergo resuscitative thoracotomy (odds ratio $[\mathrm{OR}] 2.3, p<0.01$ ) and had higher base deficits both on presentation $(-19.0$ v. $-11.6, p=0.001)$, and at the end of the index operation $(-15.9$ v. $-9.6, p=0.004)$. The chest was closed in 36 patients in a mean of $1.94 \pm 0.9$ days from the index case. Definitive chest closure was performed $1.94 \pm$ 0.9 days from the index case. Among survivors, mean base deficit normalized from $9.6 \pm 5.2$ at the end of the index operation to 1.7 \pm 5.2 before closure $(p<0.001)$. Empyema developed in 14 patients (34.1\% of $24-\mathrm{hr}$ survivors). Ten patients (24.4\%) returned to the operating room for redo thoracotomy after closure. Nine 24-hour survivors did not survive to discharge (21.9\%). Among 24-hour survivors, laparotomy (OR 0.62, $p=$ $0.01)$ and resuscitative thoracotomy (OR $0.46, p=0.01)$ were associated with predischarge mortality. Conclusion: Damage control packing after thoracic trauma allows for postoperative correction of acidosis. Nonsurvivors were more likely to have resuscitative thoracotomy and had higher base deficits. Damage control principles should be considered in management of severely injured, acidotic thoracic trauma patients.

The effects of alcohol and illegal drugs on outcomes in traumatic brain injury patients. Brent Emigh ${ }^{1}$, Pedro Teixeira ${ }^{2}$, Ben Coopwood ${ }^{1}$, Jayson Aydelotte ${ }^{1}$, Tatiana Cardenas ${ }^{1}$, Sadia $A i^{1}$, Carlos Brown ${ }^{2}$. From the ${ }^{1}$ Dell Medical School at The University of Texas at Austin, Austin, TX; and the ${ }^{2}$ Dell Seton Medical Center at The University of Texas at Austin, Austin, TX.

Background: The sedating effects of alcohol and mind-altering effects of illegal drugs can greatly influence initial assessments of patients suffering from traumatic brain injuries (TBIs). Those severely under the influence of alcohol or drugs tend to be given artificially lowered Glasgow Coma Scale (GCS) scores, which can potentially affect management. The purpose of this study was to examine the effects of alcohol and illegal drugs on outcomes in patients with moderate and severe TBI. Methods: Retrospective analysis of our institutional trauma registry from 2010 to 2017 was performed. Adults with moderate to severe TBI $(\mathrm{GCS}<13)$, Abbreviated Injury Scale (AIS)-Head score $\geq 2$, and serum alcohol and urine drug toxicology results were included. Alcohol negative (blood alcohol concentration $[\mathrm{BAC}]=0$ ) and alcohol positive $(\mathrm{BAC}>0)$ groups were compared with univariate regression. Groups were subdivided into illegal drug negative/positive (amphetamines, barbiturates, cannabis, cocaine, phencyclidine) subgroups and compared. The primary outcome was mortality. Secondary outcomes were length of stay (LOS) in hospital and in the intensive care unit (ICU). Results: We included 1061 patients in the analysis, with 777 (73\%) being male. Just over half (564, $53 \%)$ the patients made up the alcohol negative group, and 497 $(47 \%)$ in the alcohol positive group. Compared with those with negative alcohol screens, patients in the alcohol positive group were younger $(37 \mathrm{v} .43 \mathrm{yr}, p<0.001)$ and more likely to be white $(79 \%$ v. $68 \%, p<0.001)$. Patients in the alcohol positive group had an overall lower mortality ( $20 \%$ v. $28 \%, p=0.03)$. Hospital and ICU LOS did not differ between those with negative or positive alcohol screens $(15 \mathrm{~d}$ v. $15 \mathrm{~d}, p=0.40 ; 7$ d v. $6 \mathrm{~d}, p=0.31$, respectively). In the subgroup analyses, patients in the alcohol negative group did not have a significant increase in mortality if the illegal drug screen was positive $(26 \%$ v. $29 \%, p=0.59)$. Similarly, patients in the alcohol positive group did not have an increase in mortality if the illegal drug screen was also positive $(19 \%$ v. $21 \%, p=0.47)$. Conclusion: Patients with TBI and positive alcohol screens have lower rates of mortality than those who screen negative for alcohol. In contrast, the presence of drugs on a urine toxicology screen is not associated with any difference in mortality. Patients with TBI with either positive alcohol or positive illegal drug screens should not be managed any differently than those with negative screens and should not be expected to have any worse outcomes.

Emergency use of group A plasma in trauma patients at a level I trauma centre. Fessica Lie ${ }^{1}$, Philip Dazve ${ }^{2}$. From the ${ }^{1}$ University of British Columbia, Vancouver, BC; and the ${ }^{2}$ Vancouver General Hospital, Vancouver, BC.

Background: With the increasing use of massive transfusion protocols in damage control resuscitation, group $\mathrm{AB}$ universal plasma is in decreased supply. Studies have suggested the use of group A plasma as an alternative. However, the outcomes of trauma patients receiving group A plasma remain to be elucidated. The goal of this study is to evaluate the safety of the use of group A plasma in trauma patients in a North American level I trauma centre. Methods: The use of group A plasma was initiated in July 2017 at our institution. A prospectively collected database of trauma patients who received emergency release plasma between July 2016 and July 2018 was reviewed to compare outcomes of patients who received group $\mathrm{A}$ plasma to those who received group $\mathrm{AB}$ plasma. Results: Of the 40 patients identified, 23 patients received group A plasma and 17 patients received group $\mathrm{AB}$ plasma. Patient demographics were comparable in terms of age, gender, blood type, injury type, mechanism of injury and Injury Severity Score. Between the grroup A plasma and the grroup AB plasma groups, there was no statistically significant difference in length of stay (LOS; $15 \mathrm{~d}$ v. $35 \mathrm{~d}, p=0.309$ ), plasma transfused (6.05 v. 8.35 units, $p=0.54)$, intensive care unit (ICU) admission $(65 \%$ v. $71 \%, p=$ 0.720 ) or ICU LOS ( $7.67 \mathrm{~d}$ v. $8.75 \mathrm{~d}, p=0.803)$. There were no transfusion-related complications in both groups. Mortality ( $43 \%$ 
v. $35 \%, p=0.601)$ was similar between both groups. Disposition of the patients (i.e., acute care facility, rehabilitation, departure against medical advice, or return home with or without support) was similar in both groups $(p=0.649)$. Conclusion: This study supports the use of group A plasma in an emergency setting for trauma patients as a safe alternative to grroup $\mathrm{AB}$ plasma.

Glasgow Coma Scale score and neurologic exam miss high rates of brain injuries in patients presenting with a gunshot wound to the head. Fohn Tierney ${ }^{1}$, Charles Fredericks ${ }^{2}$, Lucas Da Matta ${ }^{3}$, Thomas Messer ${ }^{1}$, Fredric Starr ${ }^{1}$, Andrew Dennis ${ }^{1}$, Matt Kaminsky', Faran Bokbari'. From the 'John H. Stroger Jr. Hospital of Cook County, Chicago, IL; the ${ }^{2}$ Rush University Medical Center, Chicago, IL; and the ${ }^{3}$ Federal University of Minas Gerais, Belo Horizonte, Brazil.

Background: Clinical decision rules have been validated to predict intracranial injuries following mild blunt head injuries (Glasgow Coma Scale [GCS] score of 15 at presentation), and thus obviate the need for cross-sectional imaging of the head. There are no such tools for penetrating brain injury. We therefore sought to determine the prevalence of neurologic injury in patients who present neurologically intact following a gunshot wound to the head (GSWH). Methods: Patients who presented with a GSWH and a GCS of 15 between 2010 and 2015 were included. The primary outcome measure was the need for neurosurgical intervention. Secondary outcomes included the presence of intracranial hemorrhage $(\mathrm{ICH})$ and neurocranial fractures. Descriptive statistics and Fisher exact tests were used. Results: A total of 113 patients presented with a GCS of 15 following GSWH (mean age $29.9 \mathrm{yr}$, 95\% male). Twenty-nine (26.8\%) patients had either global or focal neurologic symptoms at presentation, most commonly loss of consciousness (LOC). Eleven patients $(10 \%)$ required neurosurgical intervention, including craniotomy (6\%), débridement (4\%), skull fracture repair (4\%), extraventricular drain placement $(1.8 \%)$, and/or craniectomy (0.9\%). Computed tomography (CT) of the head demonstrated ICH in $32(29.6 \%)$ patients and neurocranial fractures in $30(27.8 \%)$ patients. Neurological symptoms at presentation was a statistically significant risk factor for needing a neurosurgical intervention and for having $\mathrm{ICH}(p<0.05)$. However, only 7 of the 11 patients that needed a neurosurgical intervention had any neurological symptoms on presentation (sensitivity of 64\%). Among patients who had ICH, only 13 out of 32 patients related any symptoms at presentation (sensitivity of $45 \%$ ). Conclusion: Absence of neurological symptoms or history of LOC at presentation did not rule out presence of significant intracranial injuries or need for neurosurgical intervention in our patient population. Physicians should obtain a CT scan on all patients after gunshot wound to the head.

Rural trauma in northern Alberta: outcome of serial needs assessments and trauma preparedness surveys. Alyssa MacLean ${ }^{1}$, Henry Y. Fiang ${ }^{1}$, Jenny Yoon ${ }^{1}$, Michael Kim ${ }^{1}$, Sandy Widder $^{2}$. From the ${ }^{1}$ University of Alberta, Edmonton, AB; and the ${ }^{2}$ University of Alberta Hospital, Edmonton, AB.

Background: Trauma remains the primary cause of mortality in young patients in Alberta, and most patients residing in northern Alberta rely on rural emergency medical services (EMS) prehospital transport and community emergency departments (EDs) to recognize, triage and initiate resuscitation in trauma scenarios. However, rural elements of trauma care and preparedness are infrequently assessed. The purpose of this study was to conduct serial trauma needs assessments and evaluate trauma preparedness among level IV and V trauma hospitals throughout northern Alberta. Methods: Standardized surveys containing 49 questions across 9 categories were compiled using Trauma Association of Canada 2011 guidelines. Surveys were distributed to 50 level IV and $\mathrm{V}$ trauma hospitals biannually for a study period of 21 months. This study evaluated changes in survey responses from January 2017 to September 2018 for each element of community trauma care, using 2 -tailed paired $t$ tests. Subgroup analyses were also completed for level IV and V trauma centres separately. Results: Direct comparison of January 2017 and September 2018 surveys revealed overall positive response rates of $82 \%$ and $89 \%$, respectively. Two-tailed paired sample $t$ tests (with $95 \%$ confidence intervals) revealed statistically significant improvements in number of ambulances $(p=0.031)$, EMS crew certification $(p=0.024)$, care provider availability within $30 \mathrm{~min}$ utes $(p=0.044)$, ED nursing coverage $(p=0.002)$, number of ED beds $(p=0.018)$, advanced airway management equipment $(p=$ $0.024)$, crystalloid/colloid supply $(p=0.044)$, rapid infusion/ warmer $(p=0.024)$, dedicated trauma resuscitation room $(p=$ $0.044)$, universal precautions equipment $(p=0.044)$, vascular Doppler ultrasound $(p=0.024)$, warming devices $(p=0.044)$, 24-hour conventional radiology $(p=0.032)$, 24-hour computed tomography (CT) scanner $(p=0.044)$, access to family physician $(p=0.044)$, telehealth $(p=0.044)$ and teleconferencing capabilities $(p=0.044)$. Subgroup analysis of level IV trauma centres found no statistical significance. Subgroup analysis of level V trauma centres revealed statistical significance in EMS crew certification $(p=0.044)$, ED nursing coverage $(p=0.007)$, number of ED beds $(p=0.025)$, advanced airway management equipment ( $p=0.044)$, crystalloid/colloid supply $(p=0.044)$, rapid infusion/ warmer $(p=0.024)$, dedicated trauma resuscitation room $(p=$ $0.044)$, universal precautions equipment $(p=0.044)$, vascular Doppler ultrasound $(p=0.024)$, warming devices $(p=0.044)$, 24-hour CT scanner $(p=0.044)$, access to internal medicine $(p=$ $0.013)$ and family physicians $(p=0.044)$, telehealth $(p=0.044)$ and teleconferencing capabilities $(p=0.044)$. Conclusion: Serial surveillance of trauma preparedness among level IV and $V$ rural trauma hospitals in northern Alberta revealed statistically significant improvements in several elements of community trauma care over time. Frequent needs assessments highlight elements of rural trauma management that could benefit from further improvements and provide transparency for rural practitioners to identify potential gaps in their own training, resources, equipment and staffing practices that could be optimized to improve overall trauma outcomes in northern Alberta.

Performance measure to end all performance measurement. Lori Milton ${ }^{1}$, Morad Hameed ${ }^{2}$. From ${ }^{1}$ Interior Health, Kelowna, BC; and the ${ }^{2}$ Vancouver General Hospital, Vancouver, BC.

Background: Performance measurement in trauma can be a key driver of system and facility organization, accreditation, policy development, resource allocation, and budget. Still there is great variability in the literature as to key factors that reflect system performance. Often the literature focuses only on mortality. Outcomes, however, do not express the complete journey 
of a patient. When culture solely focuses on outcomes, it can miss critical influence points and opportunities to advance system design. Methods: A librarian-assisted search of the literature was performed to identify current performance measurement of trauma system integration and function. The review produced 26 exact matches and 51 articles described as close matches. After articles were screened for relevance and their references explored, 47 articles were chosen for review. Results: Eight broad themes were identified: secondary/undertriage $(n=31)$; lack of consensus and ambiguity in performance measures $(n=24)$; transfer guidelines or lack thereof $(n=23)$; transfer of the injured problematic and complex $(n=20)$; human factors, including compliance $(n=18)$, communication $(n=16)$ and education $(n=14)$; length of stay in the emergency department $(n=14)$; mortality regionalization $(n=14)$ and qualityimprovement programs $(n=14)$; and data access $(n=12)$ and inconsistency $(n=12)$. Thirty-one of the 47 articles discussed secondary triage or under-triage, which is the most commonly mentioned theme in this review. However, under-triage was not consistently linked to system performance in the way that mortality is. Outcome-based themes were most commonly mentioned as under-triage, mortality, and emergency department length of stay $(n=96)$, whereas inputs/influencing factors as themes $(n=60)$, and problem identification $(n=56)$ were less common. Inputs and influencers are undermentioned in the literature, surprisingly, as these are the key points that alter the end result. Conclusion: Triage to tertiary care is the gateway to the trauma system and is thus an ideal performance measure for system integration and function. Appropriate triage culminates from all the other themes and is the tip of a pyramid of care decisions and moments of influence. Understanding the foundation of that pyramid is essential and is the ultimate leverage hook to improving outcomes.

Second look deserves a second thought: identification of injuries following damage control laparotomy for trauma. Nori Bradley ${ }^{1}$, Curtis Wray ${ }^{2}$, Amit Agarwal', fohn Harvin ${ }^{2}$. From the ${ }^{1}$ University of Alberta, Edmonton, AB; and the ${ }^{2}$ University of Texas at Houston, Houston, TX.
Background: Expert opinion suggests second look is an appropriate indication for damage control laparotomy (DCL). The rate of identifying pathology at second look has not been described. Our objectives were to characterize findings at second look laparotomy (SLL) and to adjudicate DCL appropriateness. Methods: We performed a retrospective review of a level I trauma centre DCL database. Cases with second look as DCL indication from 2011 to 2017 were included. Findings requiring intervention at SLL were categorized as expected from index operation, unexpected, or none. Two high-volume surgeons independently reviewed operative reports to adjudicate DCL appropriateness. Cohen's $\kappa$ was used to assess interrater reliability (agreement). Results: Sixty-one of 409 DCL cases were for second look $(14.9 \%)$. Specific indications and subsequent interventions are in the table.

Table 1. Second look laparotomies

\begin{tabular}{|lcccc}
\hline Indication & $\begin{array}{c}\text { Second look } \\
\text { to assess* }\end{array}$ & $\begin{array}{c}\text { Second look } \\
\text { resulted in } \\
\text { intervention* }\end{array}$ & $\begin{array}{c}\text { Second look findings, no. (\%) } \\
(n=61)\end{array}$ \\
\hline Small bowel & $35(57 \%)$ & $9(26 \%)$ & Expected: $17(28 \%)$ \\
\hline $\begin{array}{l}\text { Colon } \\
\text { Pancreatico- } \\
\text { duodenal } \\
\text { complex }\end{array}$ & $13(21 \%)$ & $7(54 \%)$ & Anexpected: $10(16 \%)$ \\
\hline $\begin{array}{l}\text { Other } \\
{ }^{*} \geq 1 \text { assessment or intervention per case }\end{array}$ & $6(43 \%)$ & None: $40(66 \%)$ \\
\hline
\end{tabular}

Positive agreement for appropriate DCL at index operation was $37 \%$, and negative agreement was $34 \%$. Inter-rater reliability was moderate $(\kappa=0.44)$. Conclusion: Only $34 \%$ of second look DCL required intervention at SLL. Small bowel had the most frequent indication for SLL, but the lowest rate for subsequent intervention. With only moderate agreement for DCL appropriateness, injury patterns warranting SLL are currently unclear. A better determination of injury patterns to increase utility of SLL is needed. 